\title{
Doping of organic semiconductors ${ }^{\dagger}$
}

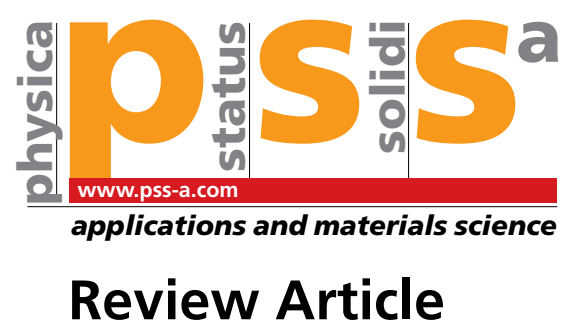

\author{
B. Lüssem, M. Riede*, and K. Leo \\ Institut für Angewandte Photophysik, TU Dresden, Germany
}

Received 14 May 2012, revised 27 October 2012, accepted 2 November 2012

Published online 20 December 2012

Keywords molecular doping, OLED, organic semiconductors, organic solar cells

*Corresponding author: e-mail moritz.riede@iapp.de, Phone: +49 351463 34389, Fax: +49 35146337065

$\dagger$ This article will be included, in edited form, as a chapter of the forthcoming book "Physics of Organic Semiconductors", edited by W. Brütting and C. Adachi (Wiley-VCH, Weinheim, 2012), ISBN 978-3-527-41053-8.

The understanding and applications of organic semiconductors have shown remarkable progress in recent years. This material class has been developed from being a lab curiosity to the basis of first successful products as small organic LED (OLED) displays; other areas of application such as OLED lighting and organic photovoltaics are on the verge of broad commercialization. Organic semiconductors are superior to inorganic ones for low-cost and large-area optoelectronics due to their flexibility, easy deposition, and broad variety, making tailormade materials possible. However, electrical doping of organic semiconductors, i.e. the controlled adjustment of Fermi level that has been extremely important to the success of inorganic semiconductors, is still in its infancy. This review will discuss recent work on both fundamental principles and applications of doping, focused primarily to doping of evaporated organic layers with molecular dopants. Recently, both p- and n-type molecular dopants have been developed that lead to efficient and stable doping of organic thin films. Due to doping, the conductivity of the doped layers increases several orders of magnitude and allows for quasi-Ohmic contacts between organic layers and metal electrodes. Besides reducing voltage losses, doping thus also gives design freedom in terms of transport layer thickness and electrode choice. The use of doping in applications like OLEDs and organic solar cells is highlighted in this review. Overall, controlled molecular doping can be considered as key enabling technology for many different organic device types that can lead to significant improvements in efficiencies and lifetimes.
1 Introduction In todays production of semiconductor devices, single crystalline silicon is by far the most frequently used material with other inorganic crystalline materials like GaAs following with a wide margin. These materials are stable and offer room-temperature mobilities up to $1000 \mathrm{~cm}^{2} \mathrm{Vs}^{-1}$. However, they are less suitable for low-cost or large-area applications. For optoelectronic applications like solar cells and light emitting diodes, silicon is also less appropriate because it is an indirect semiconductor. Therefore, silicon solar cells need rather thick material layers, resulting in higher cost, and long payback time.

A recently much-noticed alternative are organic semiconductors, i.e., molecular semiconductor consisting of carbon-based molecular entities. Early research on organic semiconductors research focused on single-crystalline organics, whose mobilities were investigated in the pioneering work of Karl et al. [1] and reach from a few $\mathrm{cm}^{2} \mathrm{Vs}^{-1}$ at room temperature to much higher values at reduced temperatures, thin-film organic semiconductor are preferred today due to practical reasons. Both of the two groups of such materials, polymers processed from solution and evaporated small-molecule compounds, are already included in present products, for example as photoconductors in copiers and laser printers. The field has been recently reviewed by several authors, we refer here to [2-4] (organic light emitting diode (OLEDs)), [5, 6] (organic electronics in general), and [7-9] (organic solar cells (OSCs)).

Organic semiconductors are superior to inorganic in the field of low-cost and large-area optoelectronics due to their broad variety, making tailor-made materials possible. For example, there are organic dyes with exceptionally high absorption coefficients in the visible range, allowing the realization of ultra-thin photovoltaic cells and photodetectors. The most important problems of inorganic LEDs like reabsorption losses can be avoided by using organic fluorescent dyes with low refraction indices and large redshifts from absorption to emission. Additionally disordered systems of organic semiconductors result in a low concentration of intrinsic defects due to their saturated electron systems: this compares to the dangling bonds of inorganic amorphous semiconductors which generate a high trap 
density. Finally, most organics are more easier to process, for example allowing deposition on flexible substrates at room temperature.

Light emission and photovoltaic effects in organics have been known for a long time. However, the possibility of organic light emitting diodes and solar cells were hardly noted by researchers until the pioneer work of Tang and VanSlyke [10, 11], who realized thin film devices by basically inserting an organic double layer between an anode and a cathode, a structure that is the fundament of both LEDs and solar cells.

Figure 1 shows schemes for OLED and OSC devices. Key property of both devices is the transport of charge carriers through thin films either from the contacts to the active zone in OLEDs or the reversed way in OSCs. It is desirable to minimize any kind of losses in this flow, so highly conductive transport layers are most suitable. Because a transition of charge carriers between the contacts and the active media is necessary for both kinds of devices, a material choice resulting in low energetic barriers or space charge layers thin enough for charge carriers to tunnel through provides the most efficient devices.

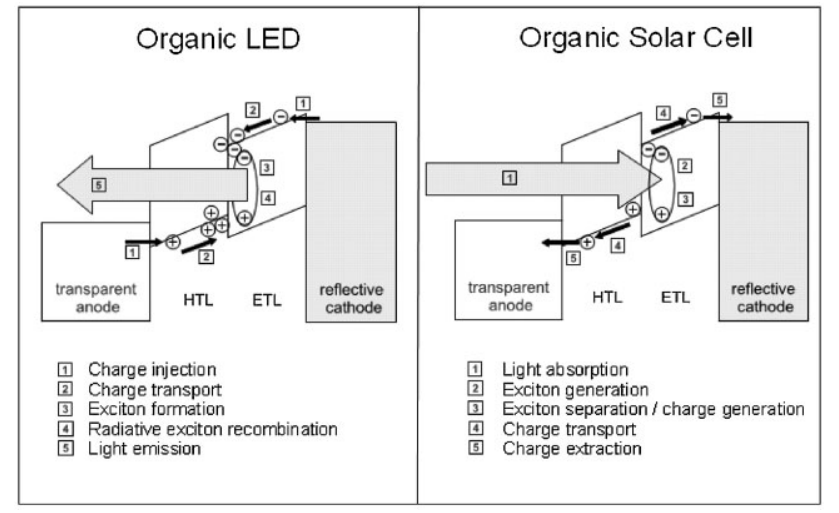

Figure 1 Operation principles of an OLED (left) and an OSC (right). Two-layer devices are shown. Reprinted with permission from Walzer et al., Chem. Rev. 107, 1233 (2007). Copyright 2007, American Chemical Society.

These two requirements, high conductivity and efficient injection, point toward the topic of this section: the possibility of controlled doping was an extremely important factor for the success of inorganic semiconductor technology

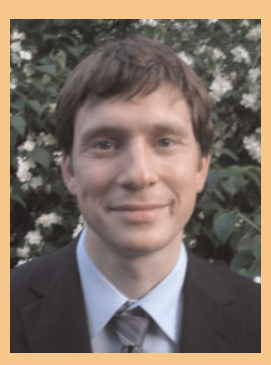

Björn Lüssem studied electrical engineering at the Rheinisch-Westfälische Technische Hochschule (RWTH) in Aachen and the University of Bath and obtained his degree as Diplomingenieur in 2003. He prepared his $\mathrm{PhD}$ thesis at the Research Center in Jülich, Germany, in the field of molecular electronics. His thesis concentrates on scanning tunneling microscopy of pure and mixed selfassembled monolayers and has been awarded the VDE-Promotionspreis and the Günther-LeibfriedPreis. After staying at the Materials Science Laboratory of Sony in Stuttgart from 2006 to 2008, he joined Prof. Leo's group at the Technische Universität Dresden where he is now the head of the New Devices Group. His main interests are new semiconducting devices based on organic materials and their differences or similarities to inorganic semiconductors.

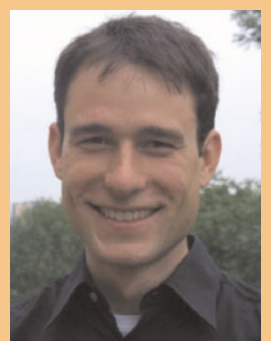

Moritz Riede studied physics at the University of Cambridge, UK, where he received his MSc degree in 2002. He obtained his PhD degree from the University of Konstanz in 2006 for research carried out at the Fraunhofer-Institut für Solare Energiesysteme and the Freiburger Materialforschungszentrum FMF. The focus of his thesis was on solution-processed organic solar cells. After his PhD he joined the research group of Prof. Leo at the Institut für Angewandte Photophysik (IAPP) of the Technische Universität Dresden in 2007, where he is now head of the Organic Solar Cell Group. His research concentrates on small-molecule-based organic solar cells, their fundamental working principles, and strategies for device optimization.

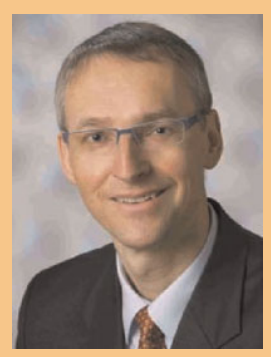

Karl Leo obtained the Diplomphysiker degree from the University of Freiburg in 1985, working with Adolf Goetzberger at the Fraunhofer-Institut für Solare Energiesysteme. In 1988, he obtained the PhD degree from the University of Stuttgart for a $\mathrm{PhD}$ thesis performed at the Max-Planck-Institut für Festkörperforschung in Stuttgart under the supervision of Hans Queisser. From 1989 to 1991, he was a postdoctoral researcher at AT\&T Bell Laboratories in Holmdel, NJ, USA. From 1991 to 1993, he was with the Rheinisch-Westfälische Technische Hochschule (RWTH) in Aachen, Germany. Since 1993, he has been full professor of optoelectronics at the Technische Universität Dresden. Since 2002, he is also with the Fraunhofer society, currently as director of Fraunhofer COMEDD. His main current interests are novel semiconductor systems such as semiconducting organic thin films, with special emphasis to understand growth, basic device principles, and the optical response. Recently, he has also worked on device development, such as highly efficient organic LED and solar cells. His work was recognized by several awards, including the Leibniz-Award in 2002. He was involved in the founding of several companies such as Novaled AG and Heliatek $\mathrm{GmbH}$. 
because it allows to take control over conduction properties. Although todays organic semiconductors are usually undoped, the prospective creation of efficient organic-based devices will require controlled and stable doping to adjust the Fermi levels to the transport states. This way Ohmic losses can be lowered while charge carrier injection is supported. Furthermore, it would enable one to raise the built-in potential of Schottky or $\mathrm{p}-\mathrm{n}$ junctions which is, e.g., helpful in OSC.

This paper discusses recent work on both fundamental principles and device applications of doping, focused primarily to doping of evaporated organic layers with a molecular dopant, using co-evaporation. In contrary to other conceivable dopants like gases or metals, molecular dopants enable very stable doping. We will begin by describing the basic physics of doping, covering both p-type [12-15] and n-type [16-19] doping. Conductivity increases of several orders of magnitude are possible in both cases, surpassing conductivities of pure materials and those caused by background impurities by far. However, the energetic positions of the orbitals in the interesting materials such as the transport layers of OLEDs and OSCs are facilitating p-type doping compared to n-type doping, making the latter a challenge for recent research. To support the understanding of the basic device principles, this paper includes some elementary model devices such as organic p-n-homojunctions, which have just been realized for the first time [20]. These structures show huge internal voltages and reveal the limits of current semiconductor models as they provide experimental data that is not compatible with the Shockley model usually used for inorganic semiconductors. We will end by discussing devices based on electrically doped organic layers, especially OLEDs, on which the application of those layers has multiple positive effects.

Decreasing the Ohmic resistance of the transport layers is a crucial for more efficient devices. Figure 2 presents a comparison between an inorganic and an organic LED. The left side shows a scheme of the band structure for a typical inorganic LED. The emitter layer has a smaller bandgap and is placed in between two transport layers, one of them being highly n-doped and the other one highly p-doped. The high conductivity of these layers results in very low Ohmic losses
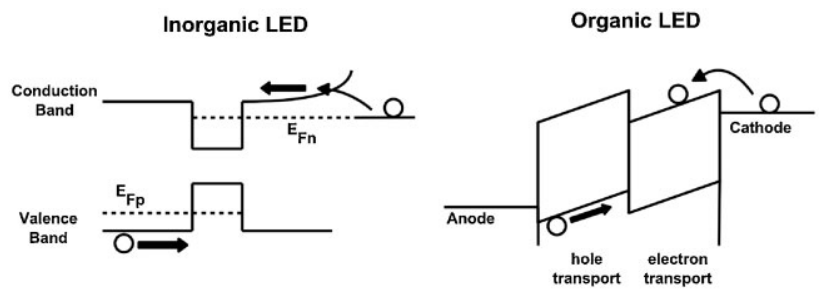

Figure 2 Left: scheme of a typical inorganic LED, which operates close to flat band condition. The injection at the contacts is by tunneling through thin space-charge layers. Right: scheme of an undoped organic LED: due to the low charge carrier concentration, a high field is needed to drive the lower number of injected carriers. Reprinted with permission from Walzer et al., Chem. Rev. 107, 1233 (2007). Copyright 2007, American Chemical Society. and almost flat band edges because of the extremely low voltage drop. Therefore, the device operates at a voltage nearly identical to the photon energy of the emitted light.

If the transport layers are not doped as in early organic light emitting diodes, the lower carrier density increases Ohmic losses and the fields necessary to drive the currents. High fields are needed since the carriers need to be injected and driven through low-conductivity layers, as shown at the right side of Fig. 2. The high losses can be reduced by very thin films, which is, however, a disadvantage for process stability. Also, the optical design of the thin-film devices profits from the thickness variations which are possible with doped layers without changing the electrical properties of the devices.

Furthermore, as shown in the left part of Fig. 2, doped transport layers generated narrow space charge regions at the contacts, allowing efficient injection by charge carrier tunneling. Despite a potentially large energy barrier between the contacts and the transport layers, Ohmic contacts are possible. This is a big advantage since it allows to avoid contact materials with unstable work functions, which would be necessary to adjusting the barriers to optimal levels, and saves additional efforts in preparation compared to OLEDs with undoped layers. The improved flexibility in material choice is of special importance to the field of top-emitting or inverted OLEDs.

Doped transport layers can also be applied in OSCs, where they result in similar advantages:

(i) They allow to virtually freely choose the thickness of doped window layers, enabling efficient optical design, i.e., for placing the optical field maxima as desired.

(ii) Since solar cells operate at very low voltages, avoiding voltage drops by creating Ohmic contacts via doping is even more crucial for efficiency than in LEDs.

(iii) In stacked cell structures, doping can significantly improve the efficiency of charge recombination at recombination contacts.

This introductory section is completed by the following list of the key materials used in the review (see Fig. 3).

\section{Doping fundamentals}

2.1 Introduction Due to the very different structure of organic and inorganic semiconductors, one can expect significant differences in the properties of doped layers. However, as we will discuss in the following section, there are many similarities in the basic properties of the doping process. Key differences in the doping behavior are caused by the fact that the transport in organic semiconductors is taking place by hopping in a distribution of more or less localized states. Additional effects are the large Coulomb correlation between charge carriers and strong polaronic coupling between localized hopping states.

The basic principle of doping is like in inorganic semiconductors and illustrated in Fig. 4. Electron donors or acceptors are added to the material, generating additional 

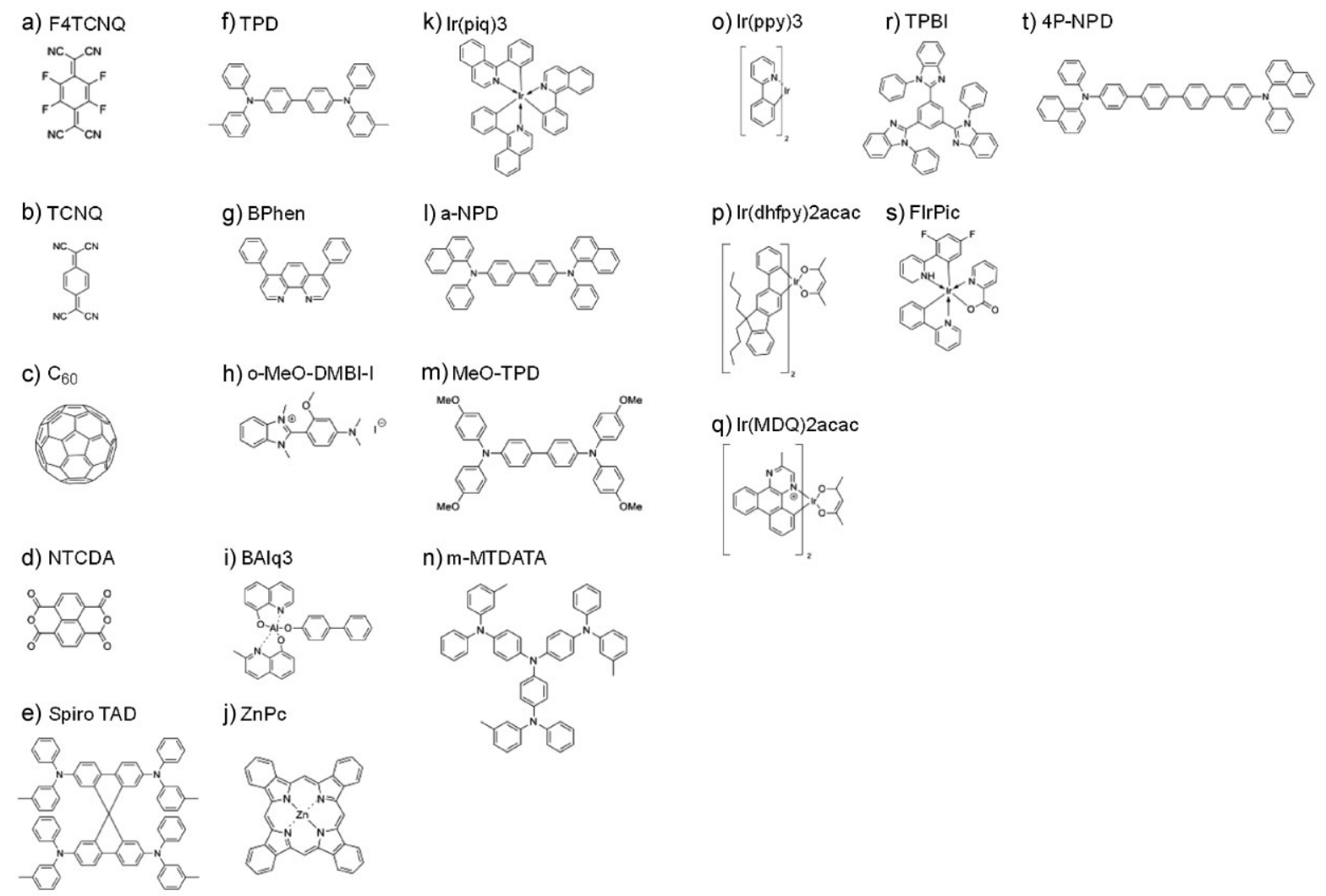

) $\mathrm{C}_{60}$

d) NTCDA

e) Spiro TAD
o-MeO-DMBI-I

BAlq3

j) $\mathrm{ZnPc}$

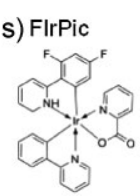

$\operatorname{Ir}(M D Q) 2 a c a c$

Figure 3 Key materials used in this paper (a) F4-TCNQ: 2,3,5,6-tetrafluoro-7,7,8,8-tetracyano-quinodimethane, (b)TCNQ: 7,7',8,8'tetracyanoquinodimethane, (c) $\mathrm{C}_{60}$, (d) NTCDA: 1,4,5,8-naphthalenetetacarboxylic dianhydride, (e) Spiro-TAD: 2,2',7,7'-tetrakis( $N, N$-diphenylamino)-9,9'-spirobifluoren, (f) TPD: $N, N^{\prime}$-bis(3-methylphenyl)- $N, N^{\prime}$-diphenylbenzidine, (g) BPhen: 4,7-diphenyl-1,10phenanthroline, (h) o-MeO-DMBI-I: 2-(2-methoxyphenyl)-1,3-dimethyl-1H-benzoimidazol-3-ium iodide, (i) BAlq: aluminum (III) bis(2-methyl-8-quninolinato)-4-phenylphenolate, (j) ZnPc: zinc phthalocyanine, (k) Ir(piq)3: Tris(1-phenylisoquinoline) iridium(III), (1) a-NPD: $N, N^{\prime}$-di(naphthalen-2-yl)- $N, N^{\prime}$-diphenyl-benzidine, (m) MeO-TPD:( $N, N, N^{\prime}, N^{\prime}$-tetrakis(4-methoxyphenyl)-benzidine, (n) m-MTDATA: 4,4', 4" -tris(3-methylphenylphenylamino) triphenylamine, (o) $\operatorname{Ir}($ ppy)3: Tris(2-phenylpyridin) iridium(III), (p) $\operatorname{Ir}(\mathrm{dhfpy}) 2$ (acac) bis(2-(9,9-dihexylfluorenyl)-1-pyridine)(acetylacetonate)iridium(III), (q) $\operatorname{Ir}(\mathrm{MDQ}) 2$ (acac): iridium(III)bis(2-methyldibenzo-[f,h]chinoxalin)(acetylacetonat), (r) TPBi: 2,2',2"'-(1,3,5-phenylen)tris(1-phenyl-1H-benzimidazol), (s) FIrpic: iridium(III)bis[(4,6-difluorophenyl)pyridinato-N,C2]picolinate, (t) 4P-NPD: $N, N^{\prime}$-di-1-naphthalenyl- $N, N^{\prime}$-diphenyl-[1, $1^{\prime}: 4^{\prime}, 1^{\prime \prime}: 4^{\prime \prime}, 1^{\prime \prime \prime}$-quaterphenyl]-4,4'"'-diamine.

mobile charge carriers. To obtain n-type doping in organics the dopant has to donate electrons to the lowest unoccupied molecular orbital (LUMO) states while p-type dopants extract electrons from the highest occupied molecular orbital (HOMO) states, therefore creating holes.

Doping in organic semiconductors using strongly oxidizing gases, e.g., iodine or bromine has been addressed
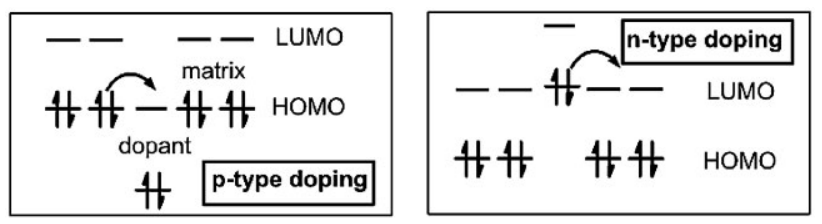

Figure 4 Scheme of the doping process for molecular p-type (left) and n-type doping (right). In p-type doping, the dopant is an acceptor, in n-type it is a donor. Adapted with permission from Walzer et al., Chem. Rev. 107, 1233 (2007). Copyright 2007, American Chemical Society. quite early: The doping of materials such as phthalocyanines lead to high conductivities [21, 167-172]. However, those dopants are too small to provide thermally stable doped layers of bipolar devices like $\mathrm{p}-\mathrm{n}-$ or $\mathrm{p}-\mathrm{i}-\mathrm{n}$ junctions due to their large tendency to diffuse.

More frequently used have been small atoms like lithium, cesium, or strontium [22, 23] and even small molecules like Lewis acids [24]. Exemplarily for the previously described effect is a report of Parthasarathy and coworkers who used ultraviolet photoelectron spectroscopy (UPS) to measure the Fermi level of the well known organic electron transporter BCP when doped with lithium. The result is shown schematically in Fig. 5, where it can be observed that the lithium ions occupy states in the BCP gap. For practical applications, the doping with ions or very small molecules usually suffers from device instability due to diffusion.

The preparation of stable devices is easier when using larger, in particular aromatic, molecules acting as good 


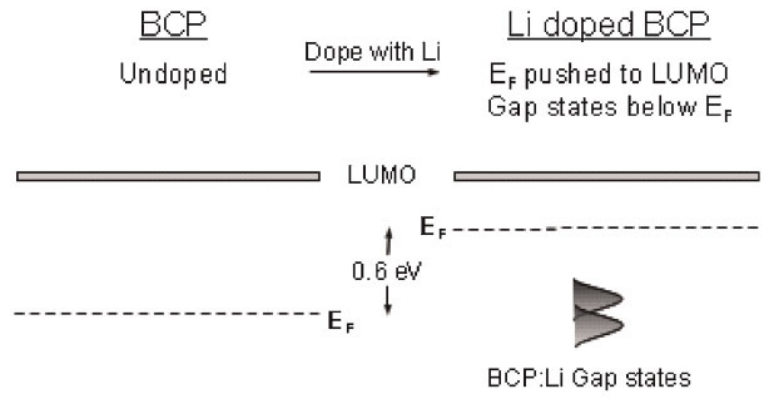

$\mathrm{HOMO}$

Figure 5 Energy levels of undoped (left) and Li-doped (right) BCP thin films. Reprinted with permission from Parthasarathy et al., J. Appl. Phys. 89, 4986 (2001). Copyright 2001, American Institute of Physics [22].

donors or acceptors. In the last decades, many possible combinations of materials and dopants have been reported, such as phthalocyanines doped by organic acceptor molecules like ortho-chloranil [25], tetracyano-quinodimethane (TCNQ) or dicyano-dichloro-quinone (DDQ) [26, 27]. The latter one has also been used to dope covalently bound stacked phthalocyanines [28] and oligothiophenes [29]. However, these dopants had usually a rather small effect. Furthermore, there was a general lack of systematic research on the impact doping has on key properties of semiconductors as the charge carrier density or the Fermi level and on device performance [26, 29].

In the last years our group has systematically investigated the physics underlying molecular doping [12, 15, 16, 20, 30-32], which lead to the development of electrically doped transport layers, that were successfully applied in both OLEDs [33-40], and OSCs [41-46]. The following sections will address our approach of doping via co-evaporation of dopants and organic matrices. Regarding p-type doping, we did extensive research on different matrix materials, including both amorphous and polycrystalline ones, that will be presented in this paper. n-Type doping is a more complicated matter as there are not many molecules combining stability with sufficiently high energy levels to reach the LUMO states of typical organic semiconductors. Nevertheless some important results will be discussed in this paper.

It should be pointed out that the microscopic understanding of the doping process of organic semiconductors is still very rudimentary. In inorganic semiconductors, there is a rather simple model, the hydrogen model, which explains most of the observations qualitatively and even to a large degree quantitatively well. Here, the doping mechanism is the release of an electron bound in a Bohr state around a charged ion core, which is basically an atom with the same electronic shell as the host (e.g., phosphor in silicon). The binding energy is then simply the hydrogen binding energy, renormalized by the dielectric constant and the effective mass of the electron or hole. This model cannot work for organic materials, due to some very simple reasons:

(i) Typically, the dielectric constant, which enters the binding energy squared, is a factor of 3-4 lower so that this already leads to binding energies which are an order of magnitude or more higher

(ii) Additionally, the effective mass in organics is expected to be significantly higher, thus causing a Coulomb binding energy which should be about two orders of magnitude higher than in inorganic materials

(iii) finally, in organics with molecular dopants, neither the host nor the dopand have the rather simply symmetry of a single atom and are also usually quite different structures, thus raising the question what the detailed microscopic arrangement is.

All these points together tend to indicate that molecular dopants in organic materials should be deep dopants and hard to understand microscopically. Surprising, while the second point is true, the first one is not. As discussed in the following sections, molecular doping of organic can be rather efficient, much more than expected for deep dopants.

In the past few years, there have been a number of attempts to better understand the microscopic origin of doping in organic semiconductors. First, we want to discuss recent work from the Koch and coworkers [164, 165] where the doping process was addressed. They showed by a combination of photoemission experiments and theoretical modeling that hybridized states between host and dopant molecules can form. The energy difference between the hybridized states and the energy transport states of the host is suggested to then reduce the doping efficiency considerably. According to those observations, a strategy for efficient doping would be to suppress the hybridization by a weaker packing of dopant and host.

Another interesting study is by Mityashin et al. [166]. In a theoretical study, the authors showed that the interaction of dopant molecules can play an important role in the doping process: by this interaction, the energy barrier for the separation of dopant and charge carrier is lowered. The interaction between dopants is more important in organic materials due to the much higher dopant concentration. The authors concluded that the doping efficiency should have a threshold at a certain dopant concentration, with low or zero doping concentration below.

It is obvious from these two examples that further studies of the doping process are needed, both experimentally and theoretically. In particular, it is necessary to know more about the local configuration of dopand and host to better understand how the initial charge transfer is accomplished.

2.2 p-Type doping p-Type doping has been shown for a wide range of different matrix/dopant combinations [47]. Both, polycrystalline and amorphous materials have 

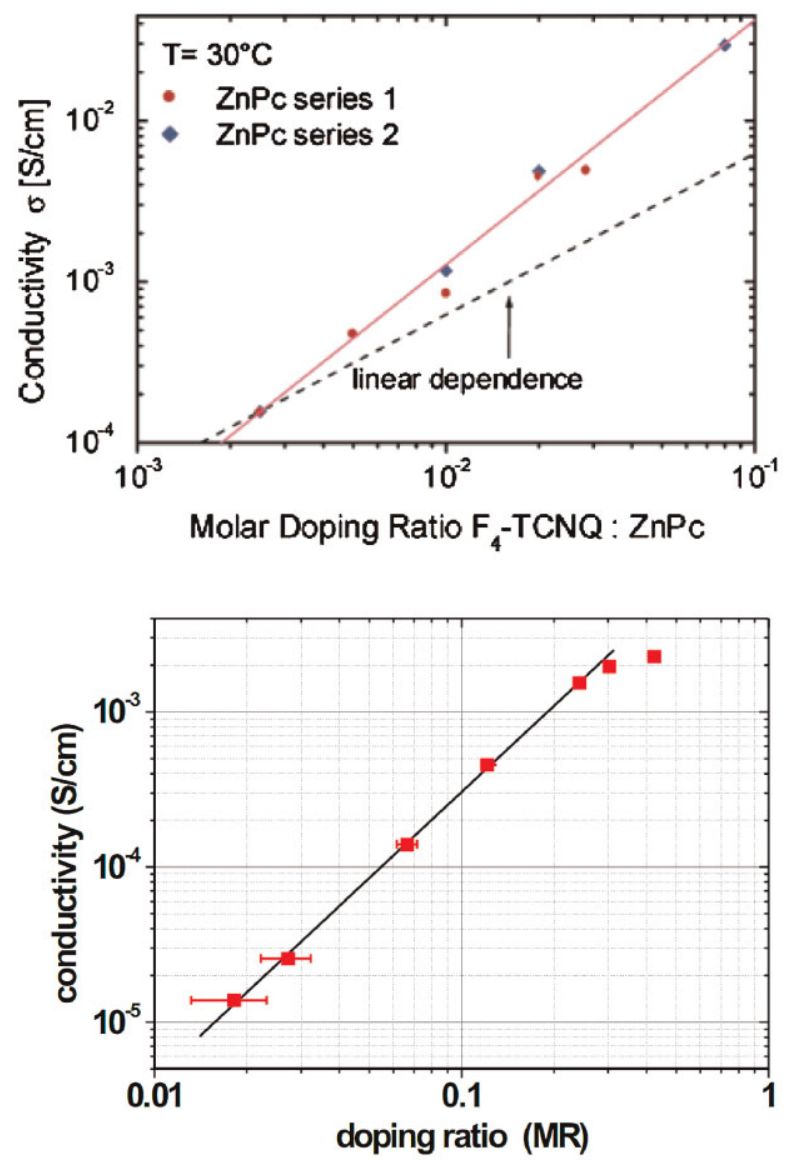

Figure 6 Doping of different host materials ( $\mathrm{ZnPc}-$ top, reprinted with permission from Walzer et al., Chem. Rev. 107, 1233 (2007). Copyright 2007, American Chemical Society [47] and MeO-TPDbottom, reprinted with premission from Olthof et al., J. Appl. Phys. 106, 103711 (2009). Copyright 2009, American Institute of Physics [32]) by the strong electron acceptor $\mathrm{F}_{4}$-TCNQ. The conductivity of both layers increases with increasing $\mathrm{F}_{4}$-TCNQ concentration.

been studied [15] and conductivities above $10^{-4} \mathrm{~S} \mathrm{~cm}^{-1}$ have been reached, which is sufficient for charge transport layers of OSCs and organic light emitting diodes.

In Fig. 6 two examples of p-doping are shown. On the top, the conductivity of a layer of a phthalocyanine, $\mathrm{ZnPc}$, doped with the strong electron acceptor $\mathrm{F}_{4}-\mathrm{TCNQ}$, is shown. Phthalocyanines are stable molecules with a low lying HOMO state and have been used as model system for many early studies of doping. On the bottom of Fig. 6, doping of an amorphous material, MeO-TPD, with $\mathrm{F}_{4}$-TCNQ is shown. $\mathrm{MeO}-\mathrm{TPD}$ is a common hole transport material in highly efficient OLEDs.

In the following, two aspects of the doping mechanism will be discussed. It is shown how doping can be used to control the Fermi-level position, which is related to the number of free charge carriers created in the organic semiconductor. Furthermore, the efficiency of the doping process, i.e., how many free charge carriers are created per dopant molecule, is discussed.
2.2.1 Control of the position of the Fermi level by doping By doping the concentration of free charge carriers is increased, or, which is equivalent, the Fermi level moves toward the transport level of the organic semiconductor.

The distance between the transport states and the Fermilevel $E_{\mathrm{F}}$ can be measured by the Seebeck effect $[12,15]$. The Seebeck coefficient $S$ depends on the distance between the transport states $E_{\mu}$ and the Fermi-level $E_{\mathrm{F}}$ :

$$
S(T)=\frac{k_{\mathrm{B}}}{e}\left[\left(\frac{E_{\mathrm{F}}(T)-E_{\mu}}{k_{\mathrm{B}} T}\right)+A\right],
$$

where $A$ is a numerical factor, which can be neglected for organic systems, $k_{\mathrm{B}}$ the Boltzmann constant, $T$ the temperature, and $e$ is the elementary charge. Seebeck measurements show that the difference between the Fermi level and the transport state decreases for increasing doping concentrations [48]. However, a discrete transport level $E_{\mu}$ is assumed in Eq. (1), which neglects the commonly observed gaussian distribution of transport states. Although correct qualitative conclusions can be drawn from these measurements, many doping induced effects cannot be understood, if a discrete transport level is assumed.

A technique, which is able to resolve the distribution of transport (HOMO) states and to determine the position of the transport states in relation to the Fermi level, is UPS. Olthof et al. [32] used UPS to study metal/p-doped organic semiconductor junctions, which lead to a deeper understanding of the doping mechanisms.

In Fig. 7a the energy level alignment of a metal/p-doped organic layer junction is sketched. After a contact between the semiconductor and the metal is established, the Fermi level $E_{\mathrm{F}}$ of the organic layer aligns with the work function of the metal $\left(W f_{\mathrm{m}}\right)$. The hole injection barrier at the interface $\phi_{0}$ is determined by the metal work function, the ionization potential of the semiconductor $I_{\mathrm{P}}$ and an interface dipole $\Delta$ $\left(\phi_{0}=I_{\mathrm{P}}+\Delta-W f_{\mathrm{m}}\right)$. Due to a charge depletion zone at the interface, the HOMO states bend upwards, and the distance between the HOMO and the Fermi level decreases with increasing distance from the interface. At the end of the charge depletion zone the distance between $\mathrm{HOMO}$ and $E_{\mathrm{F}}$, saturates at its bulk value $\phi$. All these parameters can be determined by UPS, so that a full characterization of the contact and the position of the Fermi level in the bulk is possible.

In Fig. $7 \mathrm{~b}$ the distance between the Fermi level and the HOMO states in the bulk $\phi$ of the organic semiconductor MeO-TPD is plotted versus the doping ratio of $\mathrm{F}_{4}$-TCNQ [32]. For intrinsic MeO-TPD, the Fermi level is close to the midgap $(\phi=1.68 \mathrm{eV})$. Increasing the doping ratio leads to a steep decrease in $\phi$ until it saturates at approximately $\phi_{\min }=0.35 \mathrm{eV}$.

The shift of the Fermi level at low concentrations proves that doping is effective. However, the saturation of the Fermi level shift can only be understood by a detailed analysis of the doping process. In Fig. 8a the HOMO region of the UPS 
a)
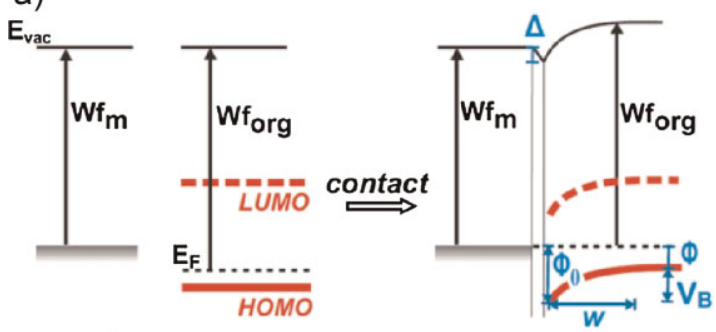

metal p-doped organic
semiconductor

b)

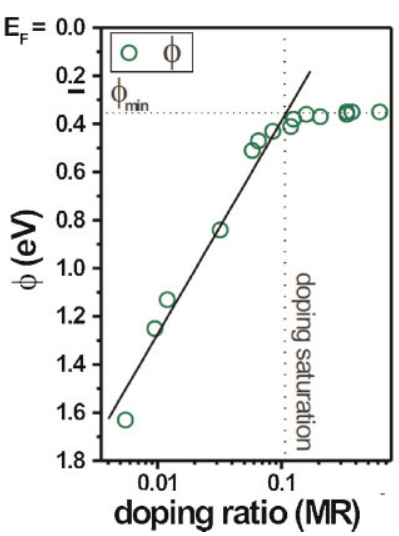

Figure 7 (a) Energy level alignment in a metal/organic semiconductor junction. In (b) the distance $\phi$ between the Fermi level and the HOMO states in the bulk of the organic layer consisting of MeO-TPD doped with $\mathrm{F}_{4}$-TCNQ is plotted as a function of the doping ratio. Silver was used as substrate. Whereas for low doping concentrations, $\phi$ decreases, it saturates at high doping concentrations. Reprinted with premission from Olthof et al., J. Appl. Phys. 106, 103711 (2009). Copyright 2009, American Institute of Physics [32].

signal of MeO-TPD doped with $\mathrm{F}_{4}$-TCNQ, and two commercially available dopants (NDP2 and $\mathrm{NDP}^{1}$ ) is shown. Although all dopants, $\mathrm{F}_{4}$-TCNQ, NDP2, and NDP9 have different doping strengths and different electron affinities, the distance between the Fermi level and HOMO states at saturation $\phi_{\min }$ does not depend on the dopant.

In contrast, in Fig. $8 \mathrm{~b}$ the matrix material has been varied. In addition to MeO-TPD, ZnPc, and PV-TPD have been doped by $\mathrm{F}_{4}$-TCNQ. It can be seen that the choice of the matrix material has a strong influence on the saturation behavior. Whereas MeO-TPD saturates at $0.35 \mathrm{eV}, \mathrm{PV}-\mathrm{TPD}$ already saturates at $\phi_{\min }=0.74 \mathrm{eV}$ and $\mathrm{ZnPc}$ at $0.2 \mathrm{eV}$.

The dependence of the saturation effect on the matrix material can be understood by the insets in Fig. 8b, which show an enlarged view of the UPS signal between the HOMO energy and the Fermi level. Commonly, the HOMO cutoff energy is defined as the intersection of a tangent and the background. However, as shown in Fig. 8b, there is a significant density of states that extents into the gap and reaches up to the Fermi level at saturation. Thus, at high doping ratios, the Fermi level is pinned by a significant

${ }^{1}$ Novaled AG
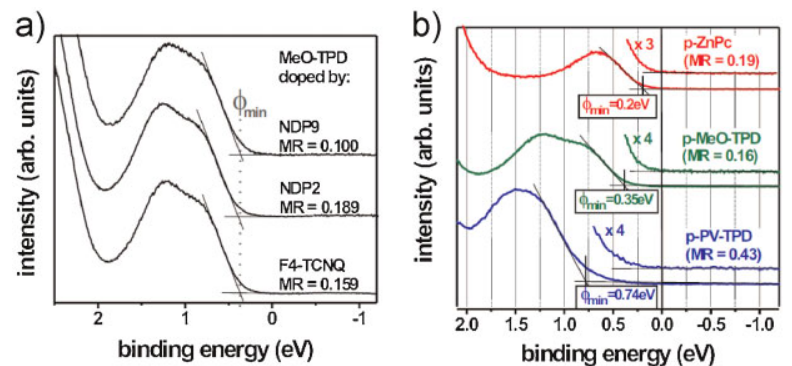

Figure 8 Part (a) shows the HOMO region of MeO-TPD doped with $\mathrm{F}_{4}$-TCNQ and the commercially available dopants NDP2 and NDP9. Although all dopants have different doping strengths, the shift of the Fermi level toward the HOMO saturates at $\phi=0.35 \mathrm{eV}$. In (b) doping of different matrix materials ( $\mathrm{ZnPc}, \mathrm{MeO}-\mathrm{TPD}$, and $\mathrm{PV}$ TPD) by $\mathrm{F}_{4}$-TCNQ is shown. $\phi_{\min }$ is strongly dependent on the matrix material. In the enlarged plots it can be seen that a significant density of states tails into the transport gap and pins the Fermi level. Reprinted with premission from Olthof et al., J. Appl. Phys. 106, 103711 (2009). Copyright 2009, American Institute of Physics [32].

density of tailing states in the transport gap, which leads to a saturation of the Fermi level shift [32].

In conclusion, by UPS it can be shown that the Fermi level is shifted toward the transport states, which implies that the number of free charge carriers in the matrix materials is increased by doping. However, up to now it has not been discussed, how efficient this process is, i.e., how many dopant molecules are ionized and how many free charge carriers are generated for a given doping concentration.

2.2.2 Doping efficiency To determine the efficiency of charge transfer between the dopant and the host molecule, Fourier transform infrared spectroscopy (FTIR) can be used. The absorption of the CN-stretching mode of $\mathrm{F}_{4}$-TCNQ depends on the charging state $Z$ of the dopant. It shifts from $2228 \mathrm{~cm}^{-1}(Z=0)$ to $2194 \mathrm{~cm}^{-1}(Z=1)$ [30].

In Table 1 the charge transfer ratio of different dopant/ matrix combinations is shown [47]. It can be seen that phtalocyanines and TDATA derivatives can be easily doped, i.e., that a complete charge transfer from the dopant to the matrix is observable $(Z=1)$. This efficient charge transfer can be explained by the electron affinity (EA) of these materials $(\sim 5.2 \mathrm{eV}[12,32])$, which is larger than the ionization energy of the matrix materials. If the EA of the dopant is reduced (e.g., TCNQ, which has an EA of only $4.5 \mathrm{eV})$, the charge transfer is much less efficient $(Z=0.2$ for the combination $\mathrm{ZnPc} / \mathrm{TCNQ}$ ). Similarly, if a host material with a higher $I_{\mathrm{P}}(e . g .$, TPD) is used, the charge transfer is less efficient ( 0.64 for the combination TPD/ $\left.\mathrm{F}_{4}-\mathrm{TCNQ}\right)$. Charge transfer for TPD derivates can be enhanced, if the $I_{\mathrm{P}}$ is lowered by electron pushing methoxy groups at the outer benzene rings, as in MeO-TPD. A charge transfer ratio of 0.74 is reached for the combination $\mathrm{MeO}-\mathrm{TPD} / \mathrm{F}_{4}-\mathrm{TCNQ}$.

Thus, the degree of charge transfer depends strongly on the EA and the ionization energy of the dopant and host molecule, respectively. However, a charge transfer ratio $Z$ of unity does not necessarily mean that one free electron is 
Table 1 p-doping of various hole transport materials by TCNQ derivates: the table shows the solid state ionization energy $I_{\mathrm{P}}$ of the matrix materials, the degree of charge transfer $Z$ from the matrix to the dopant, derived from the position of the $b_{1 u} v_{18}$ mode of the TCNQ derivates, and the conductivity $\sigma$ at a doping level of $2 \mathrm{~mol} \%$ for a series of matrix/dopant combinations [47].

\begin{tabular}{|c|c|c|c|c|c|}
\hline $\begin{array}{l}\text { matrix } \\
\text { dopant }\end{array}$ & $\begin{array}{l}\mathrm{ZnPc} \\
\mathrm{F}_{4} \text {-TCNQ }\end{array}$ & $\begin{array}{l}\text { ZnPc } \\
\text { TCNQ }\end{array}$ & $\begin{array}{l}\text { m-MTDATA } \\
\mathrm{F}_{4} \text {-TCNQ }\end{array}$ & $\begin{array}{l}\text { TPD } \\
\mathrm{F}_{4} \text {-TCNQ }\end{array}$ & $\begin{array}{l}\text { MeO-TPD } \\
\mathrm{F}_{4} \text {-TCNQ }\end{array}$ \\
\hline$I_{\mathrm{P}}(\mathrm{eV})$ & $5.1[14]$ & 5.1 & 5.1 [49] & $5.4[50]$ & $5.1[32]$ \\
\hline $\mathrm{Z}$ & 1 & 0.2 & 1 & 0.64 & 0.74 \\
\hline$\sigma\left(\mathrm{S} \mathrm{cm}^{-1}\right)$ & $1 \times 10^{-3}$ & $1 \times 10^{-6}$ & $3 \times 10^{-7}$ & $1 \times 10^{-7}$ & $1 \times 10^{-5}$ \\
\hline
\end{tabular}

generated per dopant molecule. The hole, which has been transferred to the matrix molecule still feels the attractive force of the negatively charged dopant molecule. In contrast to inorganic semiconductors, organic materials have a lower dielectric constant, which leads to stronger coulomb interactions. The attractive force between the negatively charged dopant molecule and the generated hole on the matrix is strong, and a charge carrier transferred to a matrix molecule is not necessarily free. The doping efficiency, defined as ratio of the number of free charge carriers to the number of dopants, can be below unity, even if all dopant molecules transfer charge to a matrix molecule.

To measure the density of free charge carriers in an organic semiconductor, the thickness of charge depletion zones $w$ in $\mathrm{p}-\mathrm{i}-\mathrm{n}$ and in $\mathrm{m}-\mathrm{i}-\mathrm{p}$ diodes can be measured. Olthof et al. studied the thickness of the depletion zone in $\mathrm{m}-$ i-p junctions consisting of a silver anode, an intrinsic layer of MeO-TPD $(d=5 \mathrm{~nm})$ and a p-doped layer of MeO-TPD [32] by UPS. Using the common assumption that the intrinsic layer and the charge depletion zones are fully depleted ( $p=0, p$ : density of holes), one can solve the Poisson equation

$$
\frac{\mathrm{d}^{2} \phi}{\mathrm{d} x^{2}}=-\frac{\rho}{\varepsilon \varepsilon_{0}}=\left\{\begin{array}{cl}
0 ; & 0<x \leq d \\
\frac{e N_{\mathrm{A}}^{-}}{\varepsilon \varepsilon_{0}} ; & d<x \leq d+w
\end{array}\right.
$$

where $d$ is the thickness of intrinsic layer, $\phi$ the electric potential, $\rho$ the space charge density, $\varepsilon_{0} \varepsilon$ the permittivity of the organic layer, $N_{\mathrm{A}}^{-}$the number of ionized dopants, i.e., density of dopant states that have generated a free hole, and $w$ is the thickness of depletion zone in the p-doped layer. Integration of Eq. (2) leads to an expression for $N_{\mathrm{A}}^{-}$, which equals the number of free holes $p$ in the bulk of the organic layer:

$$
N_{\mathrm{A}}^{-}=-\frac{2 \varepsilon \varepsilon_{0} V_{\mathrm{B}}}{e\left(w^{2}+2 w d\right)},
$$

where $V_{\mathrm{B}}$ is the built-in voltage [ $c f$. Fig. 7a)]. By UPS, the built-in potential and the thickness of the depletion zone can be measured experimentally for different doping concentrations. Using Eq. (3), the density of free charge carriers and thus the doping efficiency can be calculated. The result is summarized in Table 2. In addition to the analytical solution of the Poisson equation (3), the number of free charge carriers have been determined by a refined numerical calculation. At low doping densities, the doping efficiency is as low as $3-4 \%$, which means that only a fraction of dopant molecules generates free holes. At higher concentrations, the efficiency seems to increase, but the thin depletion layer thickness (below one monolayer) can lead to additional effects that are unaccounted for in this model.

2.3 n-Type doping Finding suitable materials for n-type doping is much more challenging than for $p$-type doping, but essential to realize efficient devices based on the $\mathrm{p}-\mathrm{i}-\mathrm{n}$ concept [47]. In addition to be large enough to not migrate in thin films and not to act as trap, they have to be able to add electrons to the LUMO of ideally a wide variety of matrix materials. For a direct electron transfer from a dopant, its HOMO has to lie above the LUMO of the matrix material, making n-doping fundamentally more difficult, because a high HOMO reduces the stability of the dopant against oxidation. Figure 9 shows a couple of materials and illustrates the challenges.

There are various ways for n-doping of organic semiconductors and the following three approaches will be discussed.

Table 2 Efficiency of doping MeO-TPD with $\mathrm{F}_{4}$-TCNQ at different doping concentrations. The number of free charge carriers are either determined by Eq. (3) or by a numerical simulation [32].

\begin{tabular}{lllllll}
\hline doping ratio $(\mathrm{MR})$ & $w(\mathrm{~nm})$ & $\phi_{0}(\mathrm{eV})$ & $\phi(\mathrm{eV})$ & $e V_{\mathrm{B}}(\mathrm{eV})$ & $\begin{array}{l}p\left(\mathrm{~cm}^{-3}\right) \\
\text { analytic/numeric }\end{array}$ & $\begin{array}{l}\text { ionized dopants }(\%) \\
\text { analytic/numeric }\end{array}$ \\
\hline 0.032 & $8 \pm 1$ & 1.5 & 0.84 & 0.66 & $1.5 \times 10^{18} / 2.0 \times 10^{18}$ & $3.3 / 4.3$ \\
0.065 & $5 \pm 0.5$ & 1.36 & 0.47 & 0.89 & $4.0 \times 10^{18} / 6.0 \times 10^{18}$ & $4.2 / 6.4$ \\
0.205 & $2.8 \pm 0.3$ & 1.63 & 0.38 & 1.25 & $1.2 \times 10^{19} / 1.5 \times 10^{19}$ & $3.9 / 5.0$ \\
0.370 & $0.6 \pm 0.2$ & 1.75 & 0.35 & 1.4 & $7.3 \times 10^{19} / 1.0 \times 10^{20}$ & $13.6 / 18.6$ \\
\hline
\end{tabular}




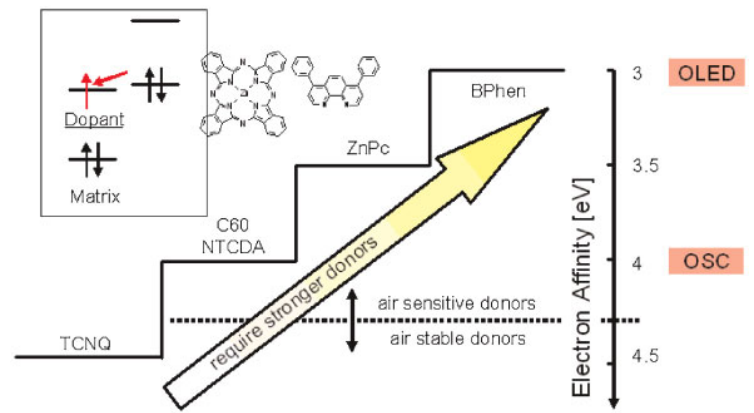

Figure 9 A series of typical electron transport materials for OSCs and OLEDs. Because the electron transport materials for OSCs typically have an $\mathrm{EA}$ of about $4.0 \mathrm{eV}$, the requirements for $\mathrm{n}$-doping materials for OSCs are more relaxed than for OLEDs. For the latter application electrons have to be added to matrix materials with an EA of about $3 \mathrm{eV}$, generally requiring materials that are increasingly requiring handling under inert conditions. Reprinted with permission from Walzer et al., Chem. Rev. 107, 1233 (2007). Copyright 2007, American Chemical Society [47].

(i) Using alkali metals.

(ii) Using molecular compounds with very high HOMO levels.

(iii) Using air-stable precursor molecules that can donate an electron to the matrix material in the deposited film.

2.3.1 n-Type doping using alkali metals The first use of n-type doping using alkali metals like potassium or sodium was reported in the 1970s [51] and subsequently investigated both theoretically and experimentally in more detail [52]. Doping using Li was first reported to dope an OLED cathode in the early 1990s [53, 54]. Li is still commonly used today and typically applied as a thin layer of pristine $\mathrm{Li}, \mathrm{Li}_{2}$, or $\mathrm{LiF}$ before deposition of the metal cathode.

The deposition of the metal cathode onto such a Licontaining layer plays an important role for the doping and the energy input from the metal deposition supports the diffusion of the $\mathrm{Li}$ into the organic layer. The most investigated interface is $\mathrm{Alq}_{3} / \mathrm{LiF} / \mathrm{Al}$, because of its frequent use in OLEDs and Fig. 10 shows the UPS studies on this interface by Mori et al. [55]. They showed that the Fermi levels align well in the presence of the $\mathrm{LiF}$ interlayer, i.e., a reduction of barrier height, leading to a good electron injection and explaining the reduced driving voltage for OLEDs.

To determine the species responsible for the doping, Hung et al. investigated this particular interface by XPS, showing that after $\mathrm{LiF}$ deposition, the $\mathrm{LiF}$ remains undissociated [56]. If $\mathrm{Al}$ is subsequently deposited onto the $\mathrm{LiF}, \mathrm{LiF}$, and $\mathrm{Al}$ were said to undergo an exothermic reaction on $\mathrm{Alq}_{3}$. However, no $\mathrm{Li}^{+}$ions were detected in their experiments [57]. Other groups obtained different results. Grozea et al. lifted off the Al electrode after its deposition and found both $\mathrm{Li}-\mathrm{F}$ and $\mathrm{C}-\mathrm{F}$ bonds in the layers underneath,

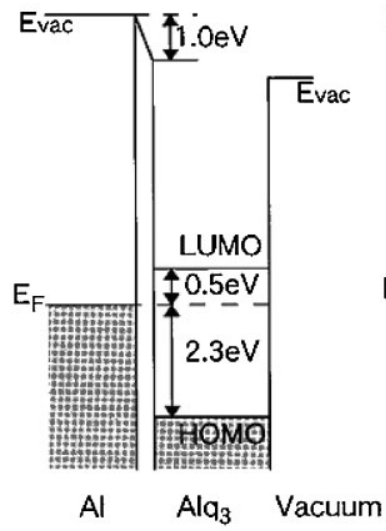

a)

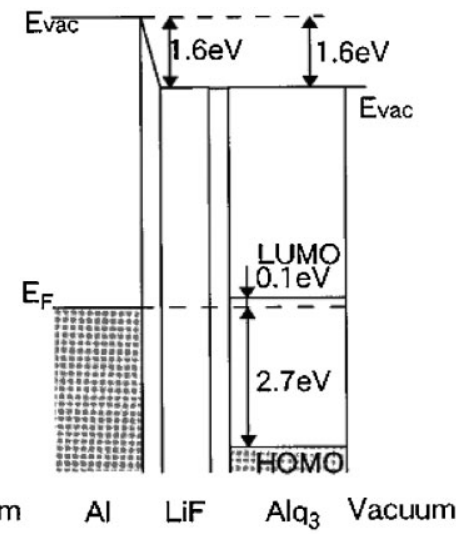

b)
Figure 10 The results of a UPS study on the interface between $\mathrm{Alq}_{3}$ $(1.7 \mathrm{~nm})$ and $\mathrm{Al}(50 \mathrm{~nm})$ without (a) and with (b) a $0.5 \mathrm{~nm} \mathrm{LiF}$ interlayer. The energy diagrams show the difference between the undoped (a) and doped (b) interface. Reprinted with permission from Hung et al., Appl. Phys. Lett. 70, 152 (1997). Copyright 2007, American Institute of Physics.

an indication that $\mathrm{LiF}$ had dissociated [58]. However, the diffusion of $\mathrm{Li}$ is not controllable, resulting in a usually unknown thickness of the doped layer. Thus, Parthasarathy et al. [22] carried out a study of Li doping from an interface layer and its diffusion into the bulk of $\mathrm{BCP}, \mathrm{CuPc}$, and $\mathrm{Alq}_{3}$, i.e., common materials for OLEDs. They found using current-voltage (I-V) measurements, UPS and secondary ion mass spectrometry (SIMS) depth profiling that they could obtain a peak conductivity of $\approx 10^{-5} \mathrm{~S} \mathrm{~cm}^{-1}$, i.e., high enough for applications, and that Li diffused nearly up to $100 \mathrm{~nm}$ into the bulk for BCP and CuPc after evaporation of a metal electrode. How much exactly Li diffuses into the bulk very much depends on the matrix and the preparation conditions, as another study determined that Li diffuses from a $0.8 \mathrm{~nm}$ layer only $10 \mathrm{~nm}$ into BPhen without the deposition of a metal electrode [59].

An alternative to n-type doping by diffusion from the interface is co-evaporating $\mathrm{Li}$ with an organic material typically at high doping ratios, leading to bulk doping. Here, the thickness of the initially doped layer is more controllable, but can lead to a good Fermi level alignment at the interface as well. Kido and Matsumoto showed efficient OLEDs with a Li-doped $\mathrm{Alq}_{3}$ or BPhen as electron injection layer [23]. Both approaches, interface and bulk doping with alkali metals are frequently used for OLEDs and the beneficial effect of an $\mathrm{LiF}$ interlayer between the organic material and the $\mathrm{Al}$ electrode was also shown for OSCs ([60], p. 215).

Other alkali metals like Cs and their salt or alloy compounds can be used for efficient n-doping of organic materials, as well. Here, Cs is often co-deposited with organic electron transport layers (ETLs) and can lead to highly efficient OLEDs [40]. Given that Cs has larger atomic dimensions, its main advantage is the reduced diffusivity as compared to Li. Thus, such devices show a higher stability 
with respect to device temperatures as Cs remains better localized in the transport layers.

However, independent of interface or bulk doping, alkali-metal ions used to generate a doped layer, still can diffuse due to their small size, which is a serious disadvantage. Their uncontrolled diffusion in the organic layers is generally detrimental to the device lifetime, as they may get into layers where it is not desired e.g. then acting as luminescence quencher in OLEDs [22, 23].

\subsection{2 n-Type doping using molecular compounds} with very high HOMO levels The first publication of controlled n-type doping using an organic molecule was presented by Nollau et al. in 2000 [16]. They showed how naphthalenetetracarboxylic dianhydride (NTCDA) could be doped by the electron donating molecule bis(ethylenedithio)-tetrathiafulvalene (BEDT-TTF). The proof of n-type doping was a clearly observable shift of the Fermi level toward the electron transport level and an increase in conductivity by $1-2$ orders of magnitude. This study showed that it is possible to n-dope organic semiconductors using molecular compounds and as such has the potential to be much more thermally stable than using alkali metals because of the reduced diffusivity. Thus, much research was carried out and several other material classes have been found.

The molecule tetrathianaphthacene (TTN, HOMO $\approx 4.7 \mathrm{eV}$ ) was shown in an UPS study to n-dope hexadecafluoro-zinc-phthalocyanine (F16ZnPc) well, but not $\mathrm{Alq}_{3}$ [61]. Given the energy levels of F16ZnPc $(\mathrm{LUMO} \approx 4.5 \mathrm{eV})$ and $\mathrm{Alq}_{3}(\mathrm{LUMO} \approx 2.5 \mathrm{eV})$ it is not surprising that TTN works more efficient as electron donor for the former matrix molecule, highlighting that the HOMO level of a dopant has to lie above the LUMO of the matrix for direct $n$-doping.

Another compound for $\mathrm{n}$-doping is the strongly reducing molecule bis(cyclopentadienyl)-cobalt(II) (cobaltocene, CoCp2) as shown by Chan et al. [62]. Their thorough investigation included conductivity measurements, X-ray studies for analyzing the structure of the vacuum deposited films, and UPS. Their matrix material was a tris(thieno)hexaazatriphenylene derivative, an electron transporter. CoCp2 has an ionization potential of only $4 \mathrm{eV}$ and was able to shift the Fermi level of their matrix material by more than $0.5 \mathrm{eV}$ toward the electron transport level, a clear proof of n-type doping. Consequently, the conductivity of the investigated films were found to increase by about three orders of magnitude, making $\mathrm{CoCp} 2$ an interesting $\mathrm{n}$-dopant and leading to further work with this material class (see Section 2.3.3).

Using metal complexes in general appears to be a good approach for n-doping, as they are also the basis for other n-type dopants. Bloom et al. suggested the compounds $\left[\operatorname{Ru}(\text { terpy })_{2}\right]^{0},\left[\mathrm{Cr}(\mathrm{bpy})_{3}\right]^{0}$, and $\left[\mathrm{Cr}(\mathrm{TMB})_{3}\right]^{0}$ as $\mathrm{n}$-dopands [63]. The electron donating character of these compounds is strong enough to dope materials used in OSCs [31], but was found to be not sufficiently n-doping for general application in OLEDs because of the higher lying LUMO values of the electron transporting materials.

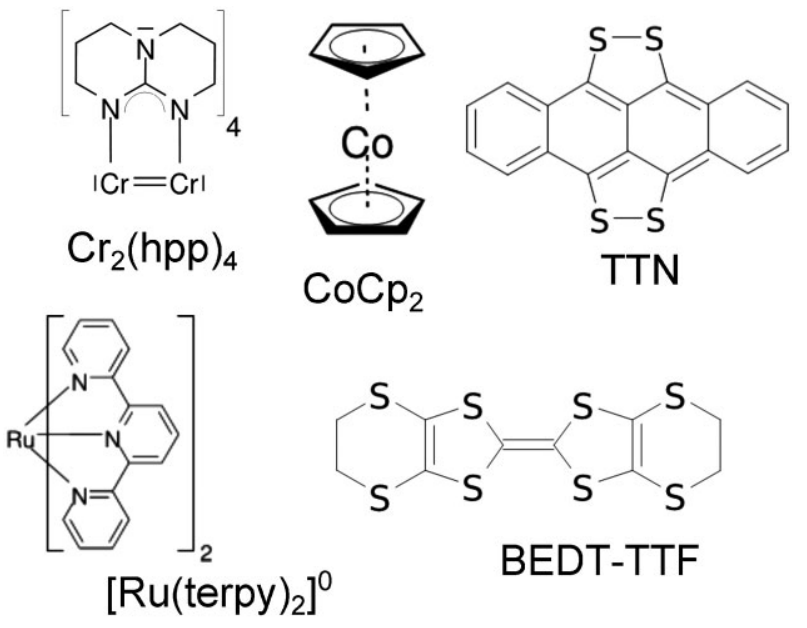

Figure 11 The chemical structures of some complexes used for direct n-doping: $\mathrm{Cr}_{2}(\mathrm{hpp})_{4}$, cobaltocene $\left(\mathrm{CoCp}_{2}\right)$, TTN, $\left[\mathrm{Ru}(\text { terpy })_{2}\right]^{0}$, and BEDT-TTF.

To directly n-dope OLED materials, dopants with higher lying HOMO are required. Novaled AG investigated two compounds first described by Cotton et al. [64-66] and recently characterized in more detail by Menke et al. [67]. The dimetal complexes of chromium or tungsten with the anion of 1,3,4,6,7,8-hexahydro-2H-pyrimido[1,2-a]pyrimidine (hpp), forming $\mathrm{Cr}_{2}(\mathrm{hpp})_{4}$ and $\mathrm{W}_{2}(\mathrm{hpp})_{4}$ (see Fig. 11) were shown to effectively dope $\mathrm{C}_{60}$ with conductivities reaching well above $1 \mathrm{~S} \mathrm{~cm}^{-1}$. It was found that $\mathrm{Cr}_{2}(\mathrm{hpp})_{4}$ doped $\mathrm{C}_{60}$ more effectively than $\mathrm{W}_{2}(\mathrm{hpp})_{4}$ although the ionization potential of $\mathrm{W}_{2}(\mathrm{hpp})_{4}$ is with $2.68 \pm 0.13 \mathrm{eV}$ even lower than Cs $(\mathrm{IP}=3.9 \mathrm{eV})[64]$. A strong shift in Fermi level toward the electron transport level was observed and the corresponding increase in conductivity is shown in Fig. 12. Furthermore, both dopants were found to lead to a stable n-doping in $\mathrm{C}_{60}$ up to $100^{\circ} \mathrm{C}$ most likely due to their molecular dimensions.

However, one general drawback of this approach for n-type doping is the increasing instability of the dopants with respect to oxygen for higher HOMO values, requiring a continuous handling of the materials under inert conditions.

2.3.3 n-Type doping using precursors Given the challenges in synthesis and handling of oxygen-sensitive substances, much research in recent years has been devoted in finding $\mathrm{n}$-dopants that do not directly dope the matrix materials, but via an intermediate form with the precursor being less or not at all sensitive to oxygen.

One possible approach is to use precursors that donate an electron to the matrix by being activated by heat or illumination. Werner et al. showed that cationic dyes like pyronin $\mathrm{B}$ chloride can be used as stable precursors for strong molecular donors [17, 18]. Figure 13 illustrates this effect of pyronin $\mathrm{B}$ chloride co-evaporated with the matrix material NTCDA where it turns into the strong donor pyronin B and the conductivity of NTCDA is increased by about four orders 

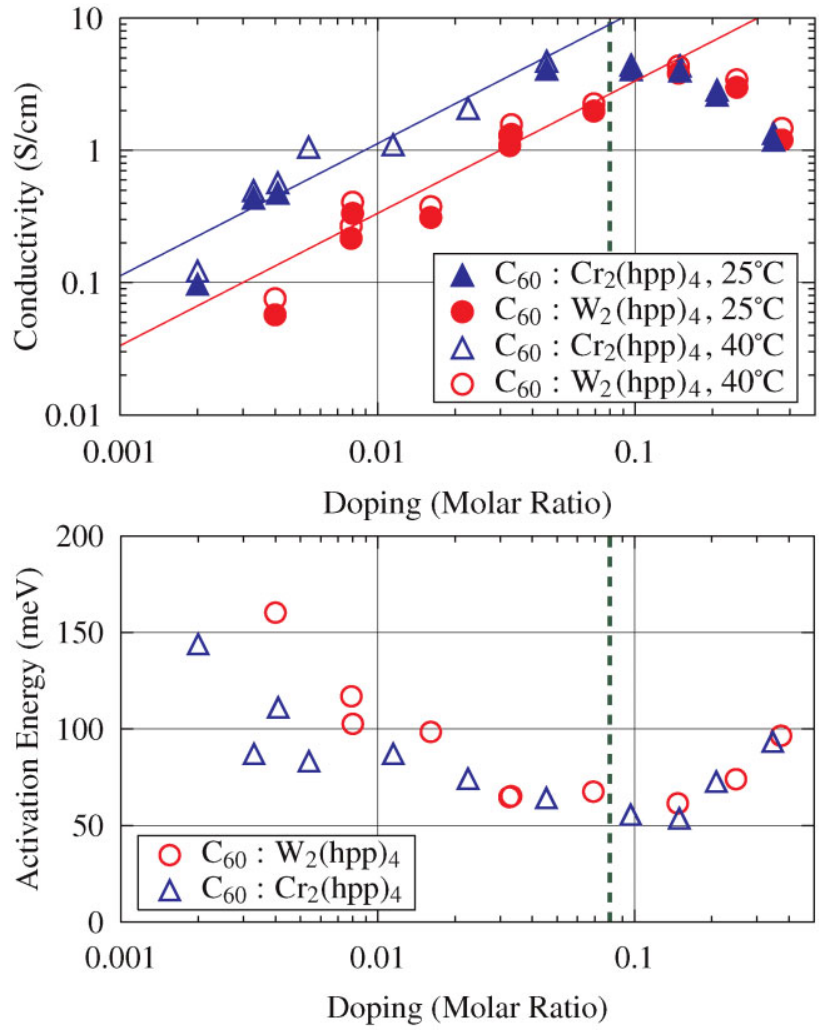

Figure 12 Lateral conductivity of $\mathrm{C}_{60}(30 \mathrm{~nm})$ at $40^{\circ} \mathrm{C}$ (top) and activation energy of the conductivity (bottom) as function of the doping concentration with either $\mathrm{Cr}_{2}(\mathrm{hpp})_{4}$ or $\mathrm{W}_{2}(\mathrm{hpp})_{4}$. Reprinted with permission from Menke et al., Appl. Phys. Lett. 100, 093304 (2012). Copyright 2012, American Institute of Physics [67].

of magnitude up to $10^{-4} \mathrm{~S} \mathrm{~cm}^{-1}$. Cationic dyes were chosen, because these molecules consist of an organic cation and an often inorganic counter ion, and as the corresponding neutral radicals have strong electron donor characters, the closedshell cations are of interest for n-type doping.

Also Chan et al. investigated the doping process of pyronin B in NTCDA in great detail, using UPS/IPES and $I-V$ measurements [68]. They confirmed the n-doping process by observing a shift in Fermi level toward the electron transport level and an increase in conductivity by more than four orders of magnitude. Combining their experimental data with calculations using density functional theory (DFT), they concluded that two species of pyronin B are present in the deposited thin film: the leuco and the neutral radical of pyronin $\mathrm{B}$, whereas they attributed the doping effect to the neutral radical.

Other stable precursor molecules are triphenylmethane cationic dyes crystal violet [19], and acridine orange base [3,6-bis(dimethylamino)acridine (AOB)] [69]. The common process is that these molecules can become in situ a volatile electron donor for n-doping of a matrix.

Further investigations using FTIR, UV/VIS/NIR, and mass spectroscopy showed that during the sublimation of triphenylmethane cationic dyes (malachite green, crystal
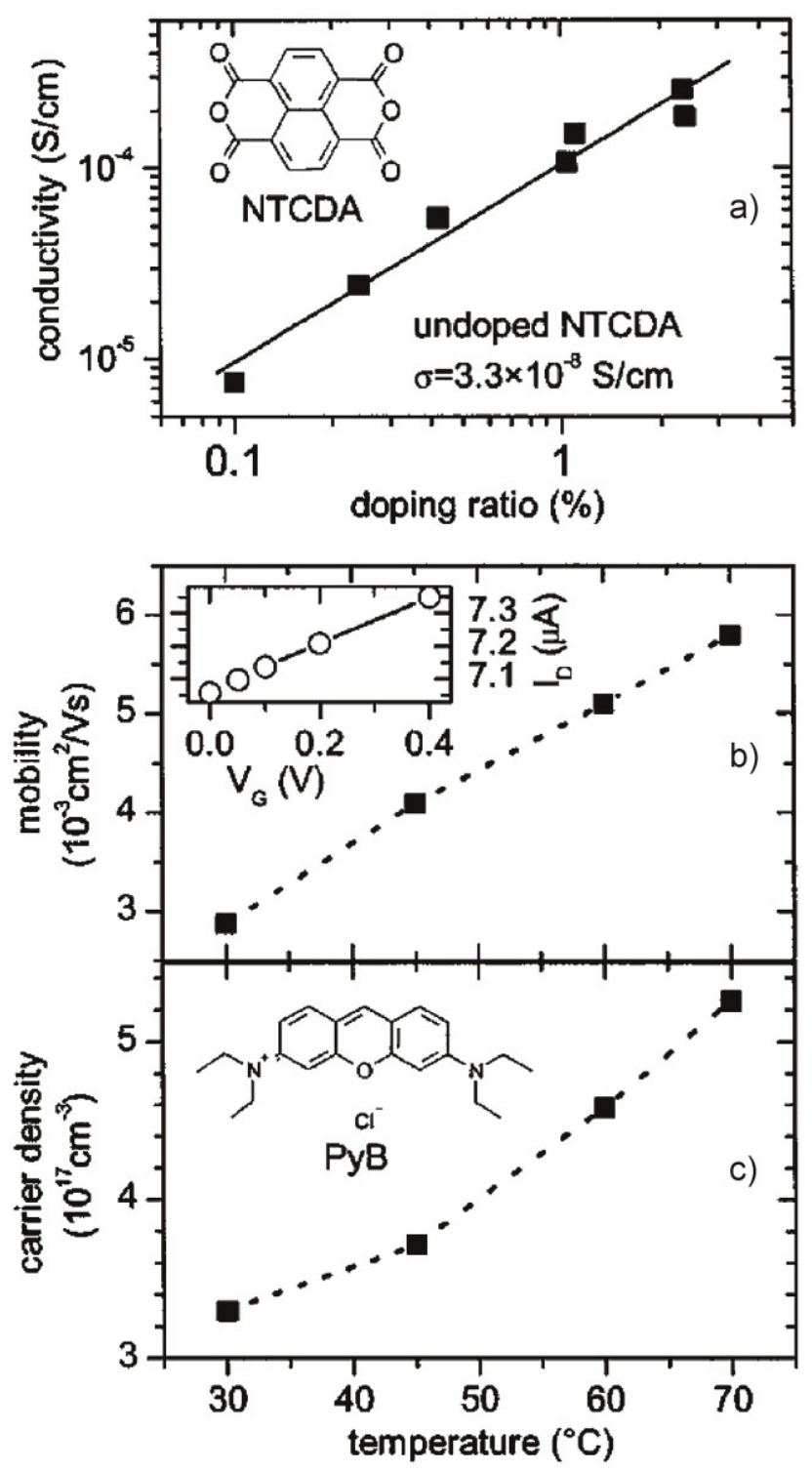

Figure 13 The conductivity of NTCDA layers vs. doping concentration with pyronin B chloride. Reprinted with permission from Werner et al., Appl. Phys. Lett. 82, 4495 (2003). Copyright 2003, American Institute of Physics. [18].

violet) and xanthene cationic dyes (pyronin $\mathrm{B}$, Rhodamine B) leuco bases are formed [17, 19]. Once the leuco forms oxidize, the original cationic dye is recreated. Using leuco bases like leuco crystal violet (LCV) and leuco malachite green the intermediate role of the leuco bases was inferred as the leuco form is only a weak electron donor, not able to directly donate electrons to most matrix materials.

The proposed mechanism of this doping process is illustrated for LCV and $\mathrm{C}_{60}$ in Fig. 14. The inital step is the illumination with light that excites LCV, i.e., lifting an electron from its HOMO to LUMO. From there, an electron transfer to the LUMO of $\mathrm{C}_{60}$ can take place, leading to an LCV cation and a $\mathrm{C}_{60}$ anion. The probability of a back 


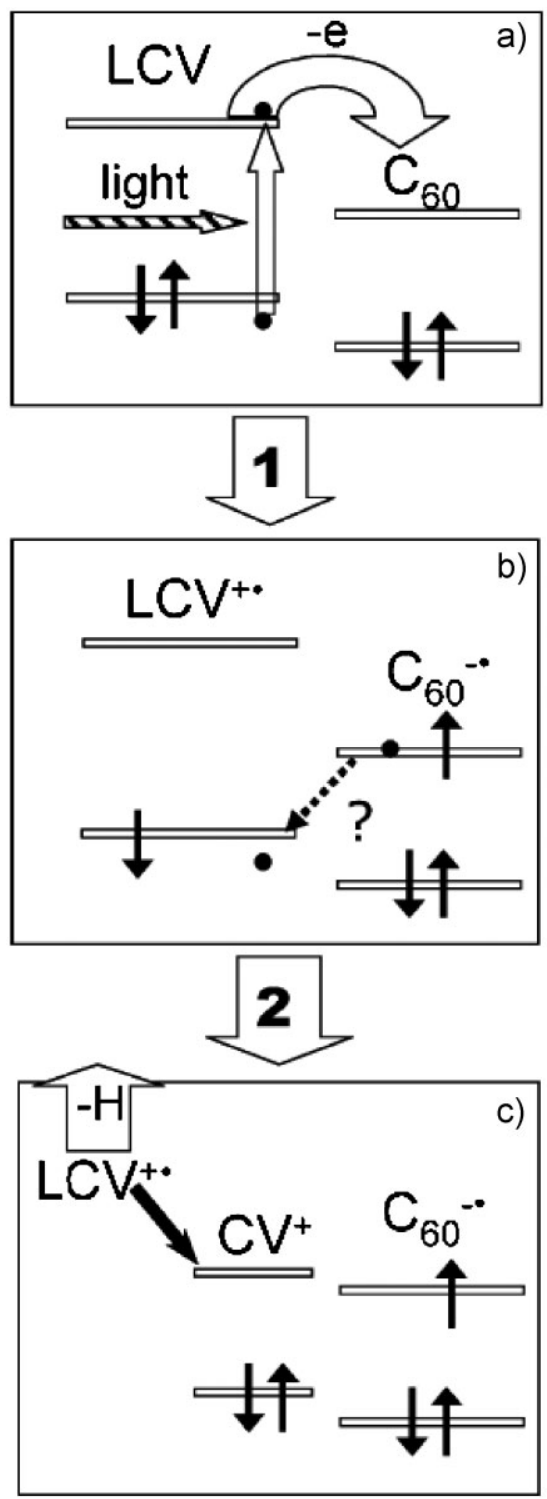

Figure 14 Schematic sketch of the proposed mechanism of the n-doping effect of LCV on $\mathrm{C}_{60}$. The n-doping effect becomes permanent by the stabilization of the positive charge on the dopant by hydride transfer to $\mathrm{C}_{60}$. Reprinted with permission from Walzer et al., Chem. Rev. 107, 1233 (2007). Copyright 2007, American Chemical Society [47].

reaction of the electron from the $\mathrm{LUMO}$ of $\mathrm{C}_{60}$ to the $\mathrm{HOMO}$ of LCV is reduced by an apparently irreversible hydride reaction of $\mathrm{LCV}$ to $\mathrm{CV}$. Through this hydride transfer the LCV with a half-filled HOMO is converted into a non-radical $\mathrm{CV}$ with a filled HOMO. As consequence, the electron back transfer from $\mathrm{C}_{60}$ is no longer possible and a stable doping has been obtained. The same effect has been observed for stronger acceptors like DDQ or TCNQ where LCV is directly oxidized to $\mathrm{CV}$ by the hydride transfer without requiring illumination to reach an excited state as it is the case for weaker acceptors like $\mathrm{C}_{60}$ [70-72].
Recently, another material class for efficient n-type doping via a precursor has been introduced by Wei et al. [73, 74]. They introduced (4-(1,3-dimethyl-2,3-dihydro1H-benzoimidazol-2-yl)phenyl)dimethylamine (N-DMBI) as a solution processable n-dopant [73] and its cationic derivative, 2-(2-methoxyphenyl)-1,3-dimethyl-1H-benzoimidazol-3-ium iodide (o-MeO-DMBI-I) as vacuum processable n-dopant [74] for fullerenes. Using o-MeO-DMBI-I they were able to obtain conductivities of more than $1 \mathrm{~S} \mathrm{~cm}^{-1}$ for vacuum deposited $\mathrm{C}_{60}$. Their hypothesis for this stable doping process was that o-MeO-DMBI-I was reduced during evaporation to its neutral radical that has a much higher SOMO level. From there, an electron transfer to the LUMO of $\mathrm{C}_{60}$ can take place, and thus the neutral radical is assumed to be responsible for the strong n-type doping effect in $\mathrm{C}_{60}$. However, the exact mechanism is still under discussion.

For OSCs doping via such precursors is frequently been used as the LUMO of $\mathrm{C}_{60}$ or other electron transport materials like NTCDA [75, 76] can effectively be doped with electrons. A low doping concentration of less than $5 \mathrm{wt} \%$ is generally enough to reach conductivities of $10^{-5} \mathrm{~S} \mathrm{~cm}^{-1}$, i.e., high enough for negligible Ohmic losses in the transport layers. However, the LUMOs of common OLED materials are too high for cationic dyes to reach a significant doping effect and consequently, this approach is hardly used for efficient OLEDs.

A novel and different approach based on air-stable dimers that can dissociated into strong n-dopants and may allow the doping of OLED materials has recently been presented by Guo et al. [77]. Whereas CoCp2 is air-sensitive, rhodocene can dimerize giving a much more air-stable compound [78]. However, when heated in vacuum, the rhodocene dimer can dissociate, resulting in a monomer with strong doping capabilities, as they illustrate for several matrix materials. Furthermore, they showed that this approach is not limited to $\mathrm{Rh}$ as metal atom for the dopant and also can be used for solution processable films.

2.4 Contacts with doped semiconductors Doping leads to an increase in the number of free charges in organic layers and thus to an increase in conductivity. However, organic devices are often limited by inefficient injection of charge carriers at the contacts. It has been tried to enhance charge carrier injection at metal contacts by the use of metals with a work function that closely matches the transport level of the organic layer, but for electron injection this often requires highly reactive metals.

Doping can also be used to optimize injection and to reduce voltage losses at the interface to metals. The energy alignment of a doped organic layer at the metal interface has already been shown in Fig. 7. Due to the difference in work function of the metal and organic, the organic layer is depleted at the interface and the remaining ionized dopant molecules generate a space charge region. According to Eq. (2) the space charge region leads to a bending of the transport states in the organic layer toward the Fermi level. The thickness of the depletion region is dependent on the 

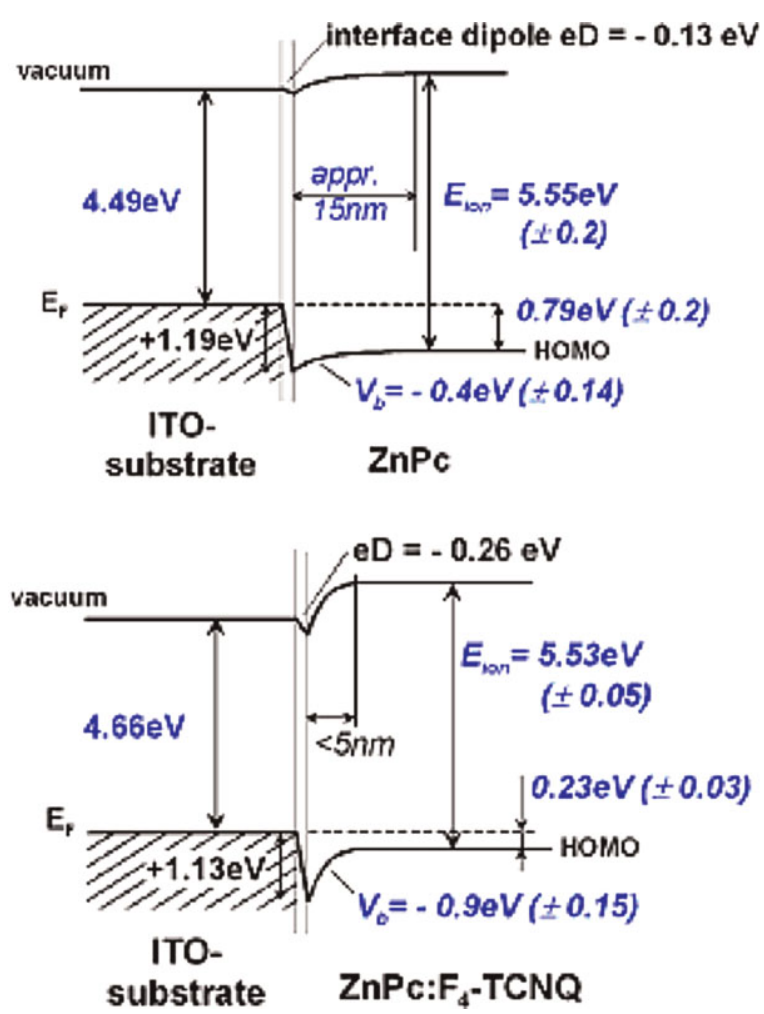

Figure 15 Bending of the HOMO level of undoped (top) and $\mathrm{F}_{4}$-TCNQ doped $\mathrm{ZnPc}$ (bottom) at the contact to ITO. Doping shifts the Fermi level toward the HOMO position, leads to a stronger level bending and a thinner injection barrier. Reprinted from Organic Electronics 2, Blochwitz et al., Interface Electronic Structure of Organic Semiconductors with Controlled Doping Levels 1997, Copyright 2001, with permission from Elsevier [14].

doping concentration (cf. Table 2). If the doping concentration is high, the transport states in the organic layer are close to the Fermi level and the depletion zone is very thin. Thus, charges can easily tunnel through the very thin injection barrier at the interface to the metal and a so-called quasi-Ohmic contact is formed.
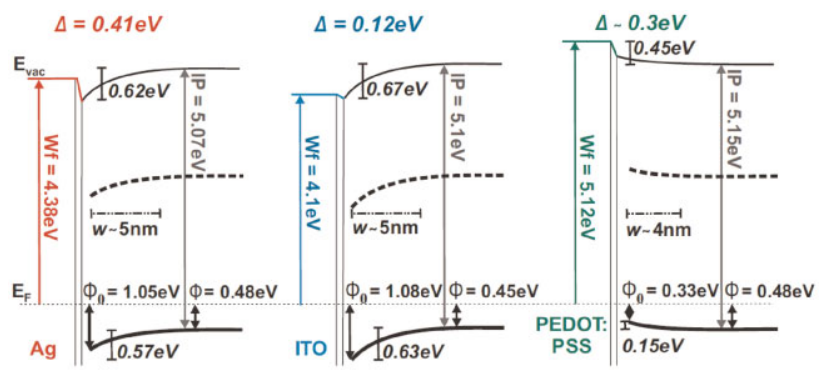

Figure 16 Energy diagram of doped MeO-TPD (4 mol\% $\mathrm{F}_{4^{-}}$ TCNQ) deposited onto Ag, ITO, and PEDOT:PSS. Despite the difference in work function of the electrode material, the difference between the Fermi level and the HOMO position in the bulk of the organic layer and the thickness of the depletion layer is almost constant. Reprinted with premission from Olthof et al., J. Appl. Phys. 106, 103711 (2009). Copyright 2009, American Institute of Physics [32].
This mechanism has been studied by UPS. In Fig. 15 the energy alignment of intrinsic $\mathrm{ZnPc}$ and $\mathrm{ZnPc}$ doped with $\mathrm{F}_{4}$-TCNQ is compared [14]. As expected, the Fermi level of the doped organic layer is shifted by $\sim 0.6 \mathrm{~V}$ compared to the intrinsic case. The level bending is stronger in the doped case and consequently the depletion zone is reduced to less than $5 \mathrm{~nm}$. Although the injection barrier is the same for both cases $(1.2 \mathrm{eV})$, the depletion zone is much thinner in the doped case leading effective tunneling and to an quasiOhmic contact.

Ohmic contacts can be realized by a wide range of electrode/organic semiconductor combinations [32]. In Fig. 16 the energy diagrams of doped MeO-TPD deposited onto Ag, ITO, and PEDOT:PSS are shown. In all cases, level bending is strong leading to a thin depletion layer and effective injection.

\section{Organic pn-junctions}

3.1 pn-homojunctions The basic element of every classical semiconductor device is a diode using a semiconductor in both its $\mathrm{p}$ - and n-doped form, i.e., a homojunction. Thus, one would assume that the same applies to organic semiconductors. However, the challenges of $\mathrm{p}$ - and n-doping for organic materials described in the previous section may explain why despite of intense research in organic semiconductors for decades it took until 2005 when Harada et al. presented the first stable and reproducible $\mathrm{p}-\mathrm{n}$-homojunction, or more precisely a $\mathrm{p}-\mathrm{i}-\mathrm{n}$ homojunction with organic materials [20]. A p-i-n structure turned out to be necessary, because given the required high doping concentration used for organic materials an intrinsic interlayer is required to achieve the blocking behavior.

There were some earlier attempts with less well defined systems [79, 80], however, they did not yield reproducible and stable $\mathrm{p}-\mathrm{n}$ homojunction. Thus, no extensive investigations on the properties or mechanisms of those structures were possible. This also implied that there was no answer whether or not the standard Shockley model p-n-junctions [81] was suitable for describing organic $\mathrm{p}-\mathrm{n}$ homojunctions. This was a particularly interesting question, because other material systems, e.g., p-n-junctions in amorphous silicon were shown to deviate from the Shockley model due to recombination processes occurring at defects that are distributed in the intrinsic layer in energy and space [82, 83].

The material choice for organic $\mathrm{p}-\mathrm{n}$ homojunctions is highly restricted by energy level requirements: p-type dopants molecules have to have an LUMO similar or even below the HOMO of the matrix (see Section 2.2) and even more challenging is finding suitable dopants for the n-doping (see Section 2.3). Given that organic molecules are typically wide gap materials, doping the same matrix with $\mathrm{p}$ - and ndoping required dopants with an extraordinary low LUMO and with a very high HOMO, respectively. Thus, in many cases heterojunctions had been chosen for devices where the realization of a $\mathrm{p}-\mathrm{n}$-(hetero)junction relaxed the requirements: the materials for $\mathrm{p}$ - and n-region can be chosen separately and in such a way to have high HOMO and low 


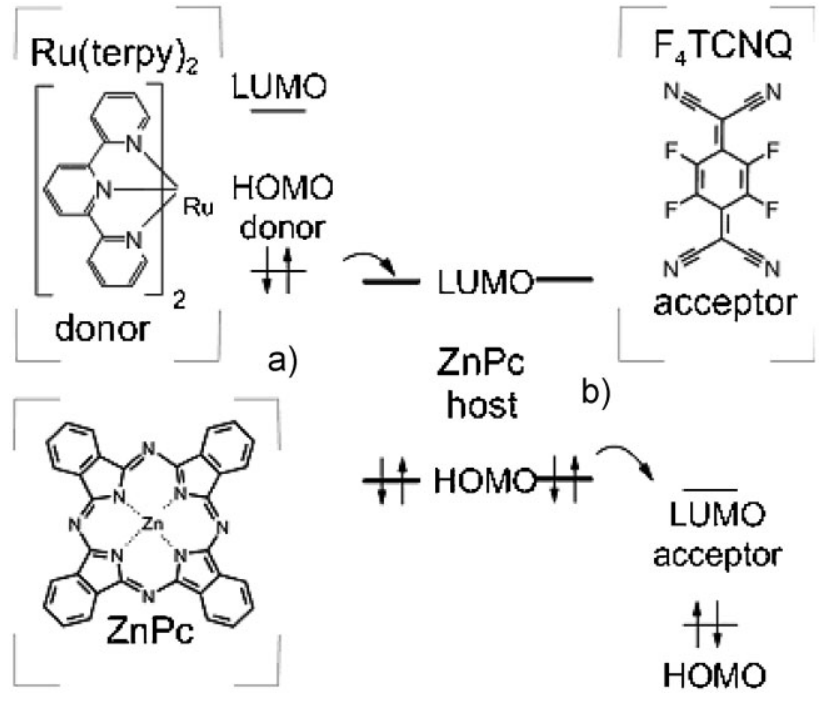

Figure 17 Materials and energy level scheme of n-type (a) and p-type (b) used to create an organic p-n-homojunction (from Ref. [20]. Copyright 2005, The American Physical Society.

LUMO, respectively, that are readily dopable with weaker dopants. However, the open circuit voltage of such heterojunctions is limited and much lower than the open circuit voltage of a real homojunction. Additionally, having a heterojunction introduces additional effects by having two dissimilar matrix materials that one wants to avoid from a scientific point of view when characterizing junction properties.

Harada et al. eventually succeeded in finding a suitable combination of $\mathrm{p}$ - and $\mathrm{n}$-dopants for an organic matrix material and their materials and a corresponding energy scheme is shown in Fig. 17 [20].

3.1.1 Experiments As described in Section 2.2, p-doping of various organic hole transport materials including $\mathrm{ZnPc}$ has been shown before $[12,15]$ where is was possible to achieve high carrier concentrations up to $10^{20} \mathrm{~cm}^{-3}$. One consequence of such a high number of charge carriers is that the space charge regions are typically very small and in the range of a few nanometers [14]. Putting $\mathrm{p}$ - and n-doped organic materials directly in contact would lead to a barrier at the $\mathrm{p}-\mathrm{n}$-junction that can easily be tunneled through and no diode-like behavior, i.e., a rectification effect would not be observed. ${ }^{2}$ Thus, so far organic diodes require instead of a direct contact between $\mathrm{p}$ - and $\mathrm{n}$-doped layer an intermediate intrinsic layer, leading to a $\mathrm{p}-\mathrm{i}-\mathrm{n}$ junction. Then, good rectification ratios can be observed and will be described for a homojunction of $\mathrm{ZnPc}$ below.

The investigated stacks of Harada et al. were ITO/ $\mathrm{p}-\mathrm{ZnPc} / \mathrm{ZnPc} / \mathrm{n}-\mathrm{ZnPc}(\mathrm{p}-\mathrm{i}-\mathrm{n})$ and as comparison ITO/ZnPc/

\footnotetext{
${ }^{2}$ Although this behavior is not desirable for diodes, it is successfully exploited in the recombination contact of organic tandem solar cells (see Section 5.2.4).
}

Table 3 Details of doping ratios and layer thickness for the investigated junctions (data from Ref. [20]) Other thicknesses of the $\mathrm{ZnPc}$ layer were also investigated.

\begin{tabular}{llll}
\hline junction type & \multicolumn{2}{l}{$\begin{array}{l}\text { doping ratio (molar)/thickness } \\
\text { of layer (nm) }\end{array}$} & \\
\cline { 2 - 4 } & $\mathrm{p}-\mathrm{ZnPc}$ & $\mathrm{ZnPc}$ & $\mathrm{n}-\mathrm{ZnPc}$ \\
\hline & & & \\
$\mathrm{p}-\mathrm{i}-\mathrm{n}$ & $1: 38 / 15$ & $-/ 30$ & $1: 46 / 40$ \\
$\mathrm{~m}-\mathrm{i}-\mathrm{p}$ & - & $-/ 30$ & $1: 41 / 100$ \\
\hline
\end{tabular}

$\mathrm{n}-\mathrm{ZnPc}(\mathrm{m}-\mathrm{i}-\mathrm{n})$ [20], where $\mathrm{p}$-doping was achieved using $\mathrm{F}_{4}$-TCNQ $[12,15]$ and n-doping using $\left[\mathrm{Ru}(\text { terpy })_{2}\right]^{0}[63]$. Table 3 summarizes the layer structures and doping parameters of the investigated devices that are sketched as insets in Fig. 18a. The characterization was carried out with impedance spectroscopy and $\mathrm{I}-\mathrm{V}$ characteristics, and the results are shown in Fig. 18.

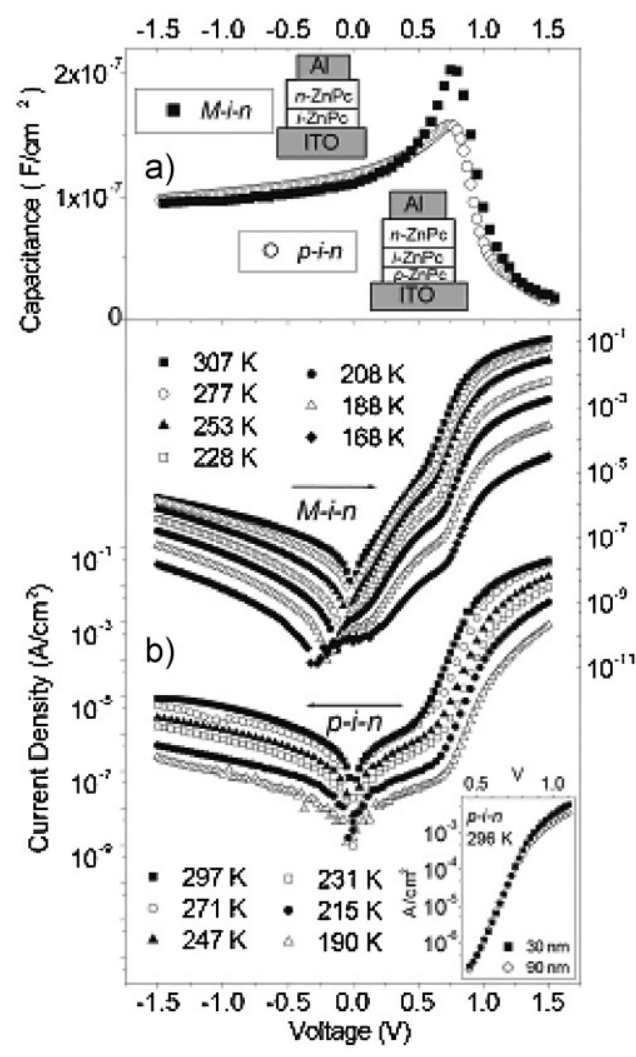

Figure 18 (a) Capacitance-voltage spectra of a $\mathrm{ZnPc} p-\mathrm{i}-\mathrm{n}$ homojunction measured at $3 \mathrm{kHz}$ and a corresponding $\mathrm{m}-\mathrm{i}-\mathrm{n}$ junction measured at $2 \mathrm{kHz}$. The insets show the stack schematics of both devices. (b) $I-V$ characteristics of the same devices at various measurement temperatures from 168 to $307 \mathrm{~K}$. The junction properties are reflected in the linear part between 0.5 and $0.8 \mathrm{~V}$. Below $0.5 \mathrm{~V}$ the characteristics are controlled by an inevitable shunt. Inset: $I-V$ characteristics of $\mathrm{p}-\mathrm{i}-\mathrm{n}$ samples with a $\mathrm{ZnPc}$ thicknesses of 30 and $90 \mathrm{~nm}$ at room temperature [20]. Copyright 2005 by The American Physical Society. 
Figure 18a shows the capacitance-voltage $(C-V)$ spectra that were used to determine the built-in potential $V_{\mathrm{bi}}$ for both $\mathrm{p}-\mathrm{i}-\mathrm{n}$ and $\mathrm{m}-\mathrm{i}-\mathrm{n}$ diodes. As the capacitance of the diodes is mainly controlled by the depletion layer width, it can be changed by different voltage bias and at a voltage equal to $V_{\text {bi }}$ the space charge region vanishes. In the experiments the capacitance collapsed in both cases at bias voltages of about $0.8 \mathrm{~V}$, implying that both $\mathrm{p}-\mathrm{i}-\mathrm{n}$ and $\mathrm{m}-\mathrm{i}-\mathrm{n}$ stack have a similar $V_{\mathrm{bi}}$ of $0.8 \mathrm{~V}$ at room temperature. This is considerably larger than the $V_{\mathrm{bi}}$ of heterojunction solar cells based on $\mathrm{ZnPc}$ and $\mathrm{C}_{60}$, but only about half of the gap expected from the onset of the optical absorption $(\approx 1.6 \mathrm{eV})$ [42]. The $0.8 \mathrm{~V}$ were also shown to be independent of the electrode as exchanging the $\mathrm{Al}\left(W_{\mathrm{f}}=4.3 \mathrm{eV}\right)$ of top contact with $\mathrm{Au}$ $\left(W_{\mathrm{f}}=5.0 \mathrm{eV} \approx W_{\mathrm{f}}(\mathrm{ITO})\right)$ lead to the same results for $V_{\mathrm{bi}}$ as well as to similar $I-V$ curves. Thus, $V_{\mathrm{bi}}$ can only be due to the difference in Fermi levels of the doped p- and n-layers of $\mathrm{ZnPc}$, independent of the metal work functions. In the case of the $\mathrm{m}-\mathrm{i}-\mathrm{n}$, the similar value of $V_{\mathrm{bi}}$ can be explained by the fact that ITO has approximately has the same work function as pristine $\mathrm{ZnPc}$ [14].

The $I-V$ characteristics taken at different temperatures in Fig. 18b appear diode-like. Both types of junctions show blocking behavior in reverse bias. In forward bias at voltages below $0.5 \mathrm{~V}$ shunts are dominating the curves. Between 0.5 and $0.8 \mathrm{~V}$ the $I-V$ characteristics are determined by the junction and show the corresponding linear increase. Above $0.8 \mathrm{~V}$ the current levels off due to the series resistance of the device. Using the standard Shockley equation for $\mathrm{p}-\mathrm{n}$ junctions

$$
J=J_{0}\left[\exp \left(\frac{e V}{n k T}\right)-1\right]
$$

where $J_{0}$ is the dark saturation current, $e$ the unit charge, $n$ the diode ideality factor, $k$ the Boltzmann constant, and $T$ is the temperature, the validity of the Shockley model for this organic homojunction was tested [20]. In Shockley theory for $\mathrm{p}-\mathrm{n}$ junctions $n$ is unity assuming only radiative recombination. In presence of recombination of charges via recombination centers located in the band gap an ideality factor between one and two is obtained. $J_{0}$ and $n$ can be fitted from the slope and the intercept of the linear part, here between approximately $0.5-0.8 \mathrm{~V}$ in the semi-logarithmic plot. However, fitting the data of Fig. 18 revealed that a temperature-dependent $n$ is required, because the slope between 0.5 and $0.8 \mathrm{~V}$ is only weakly dependent on the temperature. $n$ was found at room temperature to be between 1.8 and 2, a value that can be found for inorganic semiconductors as well. For decreasing temperatures, however, $n$ increases up to 4 as shown in Fig. 19a, but such behavior has not been observed in crystalline inorganic semiconductors and is not explicably with the Shockley model for pin-diodes.

Also the Schottky model failed to describe the observed behavior. Fitting $J_{0}$ at different temperatures to estimate the Schottky barrier, a barrier of less than half of the $V_{\mathrm{bi}}$ was

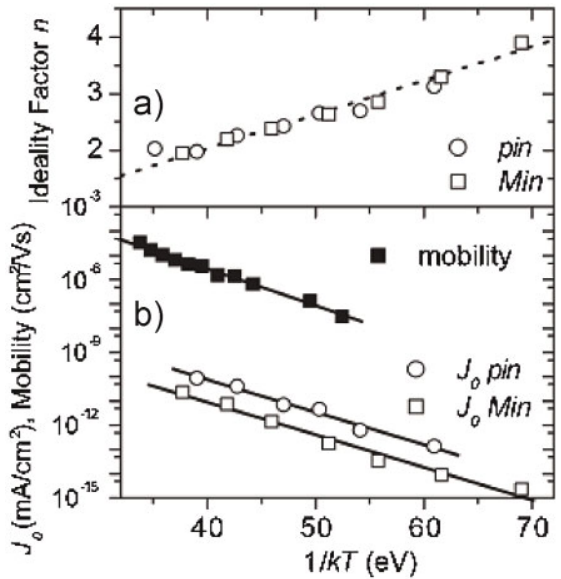

Figure 19 The temperature dependence of (a) the ideality factors $n$ and (b) the saturation current $J_{0}$ of both p-i-n and m-i-n devices. Additionally, the field-effect mobility of a $\left[\mathrm{Ru}(\text { terpy })_{2}\right]^{0}: \mathrm{ZnPc}$ (molar ratio 1:41) layer is given in (b) as comparison [20]. Copyright 2005 by The American Physical Society.

calculated and both $\mathrm{p}-\mathrm{i}-\mathrm{n}$ and $\mathrm{m}-\mathrm{i}-\mathrm{n}$ showed a similar temperature dependence. Thus, Harada et al. proceeded to suggest their own model, a changed version of the diffusion theory, that quantitatively explain the observed behavior [20]. Their central component was that deviations from the Einstein relation in disorderd systems had to be taken into account. As consequence both the 1/T temperature dependence of $\mathrm{n}$ and the thermal activation of $J_{0}$ in the exerimental data could be explained. The latter was said to be mainly determined by the temperature behavior of the mobility, because OFET measurements of n- $\mathrm{ZnPc}$ (molar doping ratio $1: 41$ ) yield an activation energy of $0.34 \mathrm{eV}$ being comparable to the slopes of $\ln J_{0}$ versus $1 / T$ for both $\mathrm{p}-\mathrm{i}-\mathrm{n}$ and $\mathrm{m}-\mathrm{i}-\mathrm{n}$ stacks (see Fig. 19).

After the initial studies and one demonstration for OSCs [31], only very recently with the availability of more materials and dopants, the concept of organic homojunctions has been investigated further. The main focus of research appears to be on OLEDs, both for solution processed [84] and vacuum processed devices $[85,86]$ with the goal to simplify the device architecture.

3.2 Reverse currents in pn-junctions In contrast to the forward characteristic of pn-junctions, the reverse currents are due to a Zener-like tunneling behavior, which can be controlled by the doping concentration of the p-and the n-layer.

The behavior of an organic pin junction consisting of aluminum $(100 \mathrm{~nm})$ as anode, $\operatorname{Ir}($ piq $) 3^{3}$ doped with NDP2 as p-doped layer, a mixed layer consisting of BAlq $2^{4}$ and NPB as intrinsic layer $(7 \mathrm{~nm}), \operatorname{Ir}($ piq $) 3$ doped with $\mathrm{NDN} 1^{5}$

\footnotetext{
${ }^{3}$ Tris(1-phenylisoquinoline) iridium(III)

${ }^{4}$ Aluminum-(III)bis (2-methyl-8-quninolinato)-phenylphenolate

${ }^{5}$ Novaled AG
} 


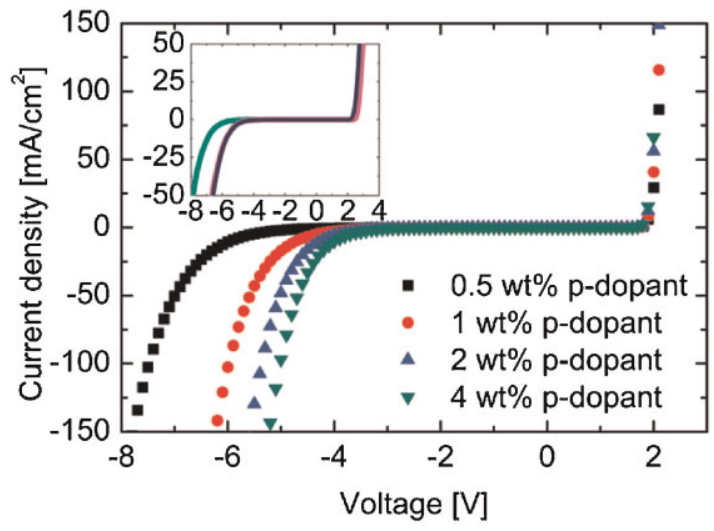

Figure 20 Organic Zener diode based on electrically doped layers. The breakdown voltage can be precisely tuned by the doping concentration of the doped layers, whereas the forward direction is not affected by the change in doping concentration. Reprinted with permission from Kleemann et al., Nano Lett. 10, 4929 (2010). Copyright 2010, American Chemical Society [87].

$(16 \mathrm{wt} \%, 50 \mathrm{~nm})$ as n-doped layer, and aluminum $(100 \mathrm{~nm})$ as cathode is shown in Fig. 20 [87]. At approximately $2 \mathrm{~V}$ the current rises sharply. At negative voltages, the device shows a reversible breakdown. The breakdown voltage is dependent on the doping concentration in the p-doped layer.

The backward current can also be controlled by the thickness of the intrinsic interlayer, as shown for a device consisting of $\mathrm{Al}(100 \mathrm{~nm}), \mathrm{MeO}-\mathrm{TPD}$ doped with $\mathrm{F}_{4}$-TCNQ ( $4 \mathrm{wt} \%, 50 \mathrm{~nm}$ ), a mixed layer consisting of BAlq $2^{6}$ and NPB as intrinsic layer $(6-10 \mathrm{~nm})$, BPhen doped Cs $(50 \mathrm{~nm})$, and $\mathrm{Al}(100 \mathrm{~nm})$ in Fig. 21. The breakdown voltage shifts by approximately $2 \mathrm{~V}$ for an increase of $2 \mathrm{~nm}$ in intrinsic interlayer thickness.

The backward current can be understood by Zener tunneling of electrons from the HOMO to the LUMO of adjacent molecules, which generates a free electron and a free hole in the n- and p-doped layer, respectively [87]. The tunneling process is dependent on the electrical field inside the intrinsic layer and the depletion zone of the pin junction. By increasing the intrinsic interlayer or the thickness of the depletion zone by lowering the doping concentration, the electrical field and the tunneling current is reduced.

This mechanism has many applications. First of all, these devices can be used as Zener diodes, which are used e.g. for overvoltage protection or as selection element in passive matrix memory structures. Furthermore, such pin junctions can be used as charge generation layers in stacked OLEDs. As will be shown in Section 4 pin diodes can connect two individual OLEDs. The pin diodes are biased in reverse direction and generate holes and electrons that are injected into the individual OLEDs. It can be shown that this process is very effective, i.e. that no voltage is lost at the charge generation unit and that the $I-V$ characteristic of the stacked unit equals the characteristic of a series connection of two individual OLEDs.

\footnotetext{
${ }^{6}$ Aluminum-(III)bis (2-methyl-8-quninolinato)-phenylphenolate
}

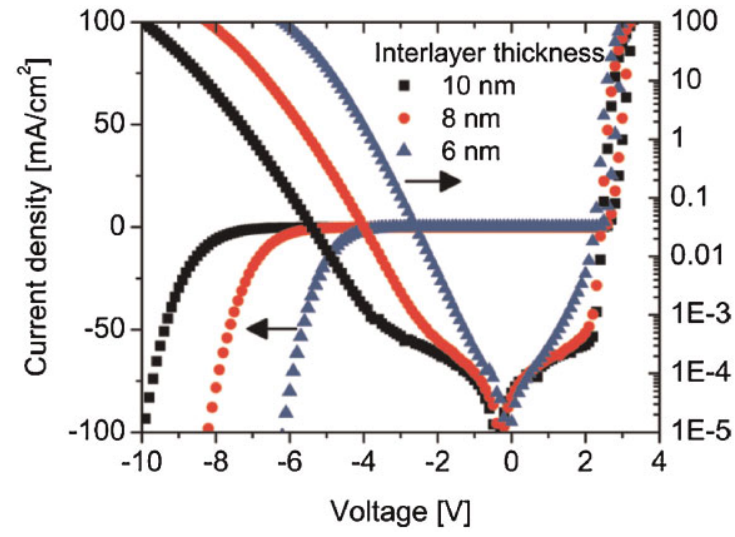

Figure 21 Control of the backward current by the intrinsic interlayer thickness of organic pin junctions [87]. The breakthrough voltage shifts by approximately $2 \mathrm{~V}$ due to an increase of the interlayer thickness of $2 \mathrm{~nm}$. Reprinted with permission from Kleemann et al., Nano Lett. 10, 4929 (2010). Copyright 2010, American Chemical Society.

4 OLEDs with doped transport layers The first observation of electroluminescence from organic single crystals stems back to the 1950s [88]. However, although these early devices showed reasonable quantum efficiencies, high voltages needed to drive current through these rather thick crystals resulting in a poor power efficiency $[89,90]$. The driving voltage was reduced significantly when thin and amorphous layers of organic materials were used instead of single crystals [91, 92]. A breakthrough was achieved when Tang and VanSlyke used an organic heterojunction consisting of an organic hole transporter and an organic electron transporter [11]. Tang and VanSlyke were the first to reach a luminous efficacy beyond $11 \mathrm{~m} \mathrm{~W}^{-1}$.

Since then, progress in the field has been vast. Further milestones in OLED development were the use of phosphorescent emitters that utilize all excitons formed electrically in the OLEDs [93] and the use of electrically doped transport layers that lead to driving voltages close to the thermodynamic limit [94]. Nowadays, monochrome OLEDs with external quantum efficiencies beyond 50\% [95] and white OLEDs with power efficiencies beyond the efficiency of fluorescent tubes have been reported [40], which has lead to significant interest from the industry.

OLEDs are currently used as active matrix displays by many handheld devices. Furthermore, several companies such as Sony and LG have developed large OLED TV sets. Beside display applications, another future market of OLEDs is the lighting industry. OLEDs are highly efficient, can produce high quality white light and are a large area light source, which sets OLEDs apart from other lighting technologies. Currently, demonstrator kits of white OLEDs are sold by Philips ${ }^{7}$ and OSRAM ${ }^{8}$.

\footnotetext{
${ }^{7}$ https://www.lumiblade-shop.com/

${ }^{8} \mathrm{http}: / /$ www.osram-os.com/osram_os/EN/Press/Press_Releases/ Organic_LED/ORBEOS-OLED-light-source.html
} 
4.1 Efficiency of OLEDs In the past, OLED research was mainly driven by the need to further improve the efficiency of OLEDs. To optimize the efficiency, all elementary processes involved in photon generation have to be understood and improved.

The working mechanism of a simple OLED is shown in Fig. 22. A single organic layer described by its HOMO and LUMO energy is enclosed by two contacts, an anode, which often consists of the transparent conductive oxide ITO deposited onto a glass substrate, and a cathode consisting of a thin layer of metal. The contacts are depicted by their work function $\Phi_{\mathrm{C}}$ and $\Phi_{\mathrm{A}}$.

Electrons and holes are injected at the cathode and anode (1), they drift toward the middle of the layer due to the applied electrical field (2), and recombine and form an exciton (3). To generate light, the exciton has to decay to the ground state and to emit a photon (4). However, many photons generated inside the OLED are trapped inside the planar device and only a fraction of the photons are extracted (5).

4.1.1 External quantum efficiency $\eta_{\mathbf{q}}$ The external quantum efficiency $\eta_{\mathrm{q}}$, which is the ratio of the number of emitted photons to the number of injected charge carriers (either holes or electrons), can be described by [96]:

$$
\eta_{\mathrm{q}}=\gamma \chi \int_{\lambda} \eta_{\mathrm{r}}(\lambda) \xi(\lambda) s_{\mathrm{PL}}(\lambda) \mathrm{d} \lambda
$$

where $\gamma$ denotes the electrical efficiency or charge balance, $\chi$ the exciton spin factor, $\eta_{\mathrm{r}}$ the efficiency of radiative decay, $\xi$ the outcoupling efficiency, and $s_{\mathrm{PL}}(\lambda)$ the normalized photoluminescence spectrum of the emitter. Both, the efficiency of radiative decay and the outcoupling efficiency are wavelength dependent.

Processes (1) to (3) of Fig. 22 are usually described by the so called electrical efficiency or charge carrier balance $\gamma$, which is defined as the probability that an injected charge carrier finds a charge of opposite polarity and forms an exciton. The charge carrier balance $\gamma$ is a measure for

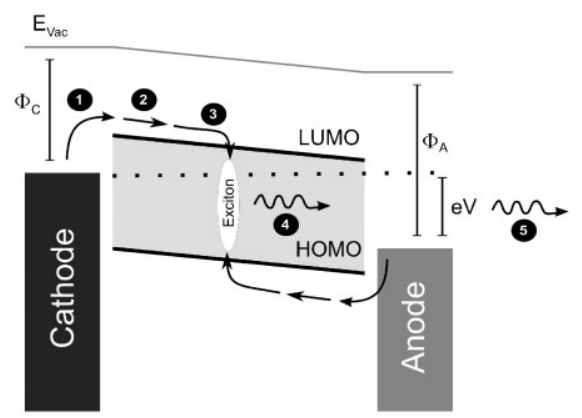

Figure 22 Working mechanism of a simple OLED. Charges are injected into the organic layer (1), are transported to the middle of the layer (2), where they recombine and form an exciton (3). The exciton relaxes to the ground state and emits a photon (4), which is finally extracted from the planar device (5). the bipolar character of the device. If the same amount of electrons and holes is injected into the device, the charge carrier balance is unity, all charges find a charge of opposite sign and can form an exciton. If there is an excess of one type of charge carrier and either more holes or more electrons are injected into the device, not all charges contribute to exciton formation and hence light emission.

Process (4), the emission of a photon, is influenced by two factors, the exciton spin factor $\chi$ and the probability of radiative decay $\eta_{r}$. In OLEDs, two types of excitons are formed: singlets and triplets. Due to spin statistics, approximately three triplets per singlet are formed. In fluorescent emitters, the transition of the molecules from the triplet state to the ground state is spin-forbidden, limiting the efficiency of these emitters or the exciton spin factor $\chi$ to 0.25 . Using phosphorescent emitters, all excitations of the molecule are directed to the triplet state, which, due to a strong spin-orbit coupling, can relax into the ground state. Thus, both, triplets and singlets, take part in the emission process and the exciton spin factor is unity.

Even though all excitons can take part in the emission process, the radiative decay into the ground state always competes with non-radiative relaxation processes. The efficiency of radiative decay $\eta_{r}$ is defined as

$$
\eta_{\mathrm{r}}=\frac{\Gamma_{\mathrm{r}}}{\Gamma_{\mathrm{r}}+\Gamma_{\mathrm{nr}}},
$$

where $\Gamma_{\mathrm{r}}$ and $\Gamma_{\mathrm{nr}}$ denote the rate constants of radiative and non-radiative decay of excitons. The rate of radiative decay $\Gamma_{\mathrm{r}}$ of the molecular emitter highly depends on its optical environment. Due to the difference in the refractive index of the organic layers and ITO $(n \approx 1.7-1.8)$, the glass substrate $(n \approx 1.5)$, and air, light is reflected at the interface between organic layers and glass and between the glass substrate and air (see Fig. 23). Furthermore, as photons are also reflected at the metallic cathode, the OLED essentially forms an optical cavity. Depending on the strength of the cavity, the radiative rate is altered by the factor $F(\lambda)$ (Purcell effect)

$$
\Gamma_{\mathrm{r}}=F(\lambda) \Gamma_{\mathrm{r}, \mathrm{inf}},
$$

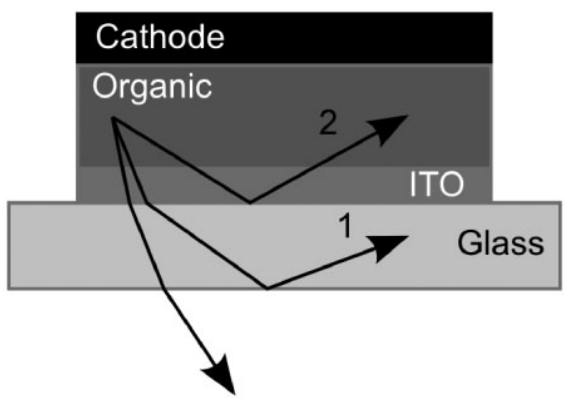

Figure 23 Due to the difference in refractive index of the organic layers including ITO, glass, and air, total reflection occurs at the interfaces. Light generated inside the OLED cavity is trapped in organic (2) and glass (1) modes. 
where $\Gamma_{\mathrm{r}, \text { inf }}$ is the radiative rate in an infinite medium, i.e., if all cavity effects can be neglected. The efficiency of radiative decay can therefore be refined to incorporate cavity effects:

$$
\eta_{\mathrm{r}}=\frac{F(\lambda) \Gamma_{\mathrm{r}, \mathrm{inf}}}{F(\lambda) \Gamma_{\mathrm{r}, \mathrm{inf}}+\Gamma_{\mathrm{nr}}}=\frac{q F(\lambda)}{q(F(\lambda)-1)+1},
$$

where $q$ denotes the efficiency of radiative decay in an infinite medium.

Finally, the outcoupling efficiency $\xi(\lambda)$ quantifies process (5) of Fig. 22. Even though a photon has finally been generated, it can be trapped inside the OLED cavity and is hence lost for light generation. Photons that are reflected at the interface between organic layers and glass are feeding waveguided modes, the so called organic modes (arrow 2 in Fig. 23), and light reflected at the interface between glass and air is lost in so called glass modes (arrow 1 in Fig. 23). The probability that a photon, which was generated inside the OLED is extracted from the cavity is given by the outcoupling efficiency $\xi(\lambda)$. Simplifying calculations show that for a normal planar device the outcoupling efficiency is in the range of $20 \%$ [97].

4.1.2 Power efficiency or luminous efficacy The external quantum efficiency alone is not sufficient to characterize the efficiency of an OLED, as it only accounts for the number of injected charge carriers and the number of emitted photons, but does not take into account their energy. An energy balance is given by the power efficiency or luminous efficacy $\eta_{\mathrm{p}}$ :

$$
\eta_{\mathrm{p}}=\eta_{\mathrm{q}} \frac{\overline{h v}}{e V}
$$

Here, $\overline{h v}$ denotes the average energy of an emitted photon and $e V$ the energy of the injected charge carriers. For displayand lighting applications the emitted light output is often weighted by the sensitivity of the human eye. Thus, the power efficiency is given in photometrical quantities $\left(\operatorname{lm} \mathrm{W}^{-1}\right)$.

4.2 $\mathbf{p}-\mathbf{i}-\mathbf{n}$ OLEDs The OLED presented in Fig. 22 is simple, but for highly efficient OLEDs at least five different layers are needed ( $c f$. Fig. 24): an ETL and a hole transport

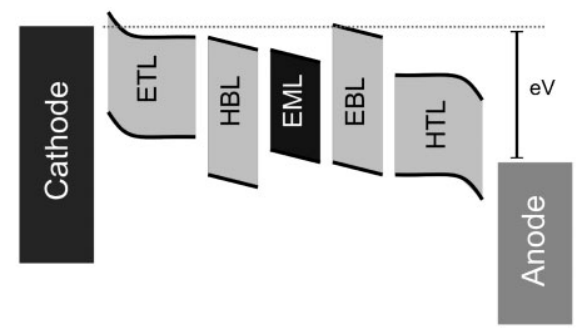

Figure 24 Five layer $\mathrm{p}-\mathrm{i}-\mathrm{n}$ OLED. The OLED consists of ETL and HTL, EBL and HBL, and an EML layer (HTL), which are electrically doped, an emission layer (EML), where excitons are generated, and finally two charge blocking layers (electron blocking layer, EBL, and hole blocking layer, HBL), which confine all charges inside the EML.

In this so called $\mathrm{p}-\mathrm{i}-\mathrm{n}$ setup, both, the quantum and the power efficiency, are optimized [47,96]. The driving voltage of the OLED is reduced by the doped transport layers. Doped transport layers improve the charge carrier injection at the contacts. As discussed in Section 2.4, a very thin depletion zone is generated at the contact to the electrodes, which can be easily tunneled through. Thus, an Ohmic contact is established, which avoids a large voltage drop at the contacts. Furthermore, due to the high conductivity of the doped transport layer, charges are efficiently transported toward the EML, which results in low driving voltages and high power efficiencies.

The charge carrier balance $\gamma$ is optimized by the EBLs. Once an electron or a hole has been injected into the EML, it is confined in the EML by a large offset in the transport level of the blocking layer. Furthermore, the blocking layers separate the EML from the doped transport layers and therefore avoid quenching of excitons.

The exiton spin factor $\chi$ and the efficiency of radiative decay $\eta_{r}$ are optimized by the choice of the EML. First of all, to maximize the exciton spin factor, phosphorescent emitters have to be used. As these emitters usually suffer from so called concentration quenching if used as bulk layer [98, 99], they are doped at low concentrations into a suitable matrix. To optimize the efficiency of radiative decay, emitters with a high photoluminescence efficiency have to be chosen. However, as discussed above, the efficiency of radiative decay of the emitters will also be influenced by the optical cavity of the OLED.

Doping allows to optimize the optical cavity of the OLED and therefore improves the outcoupling efficiency $\xi$. To obtain efficient emission of light from the OLED, the resonance, i.e., the thickness of the OLED has to be tuned to the wavelength of the emitted light and the emitters have to be placed into the antinode of the optical field inside the OLED. Doping provides sufficient free charges in the doped layer to reach a conductivity above $10^{-4} \mathrm{~S} \mathrm{~cm}^{-1}$, which is much higher than the conductivity of the undoped EMLs. Thus, almost all voltage drops across the thin EMLs and not across the charge transport layers, and the thickness of the charge transport layers can be used to optimize the optical cavity without introducing additional voltage losses. An example of an optimization of OLED efficiency by variation of the electron transport layer thickness will be discussed in Section 4.2.1. Besides the optimization of the stack design of the OLEDs, there are many other techniques to optimize the outcoupling efficiency [100]. The most successful techniques are scattering layers inside the OLED, microlens foils attached to the glass substrate or the use of index matched glass substrates. Some of these techniques are discussed in Sections 4.2.1 and 4.2.2. 


\begin{tabular}{|c|}
\hline Ag (100) \\
\hline $\begin{array}{c}\text { Bphen (60) } \\
\text { Cs }\end{array}$ \\
\hline Bphen/BAlq (10) \\
\hline NPB: \\
\hline SpQ $)_{2}$ (acac) (20) \\
\hline Spiro-TAD (10) \\
\hline NHT5 (50) \\
NDP-2 \\
\hline ITO (90) \\
\hline Low-n (LI) glass \\
\hline
\end{tabular}

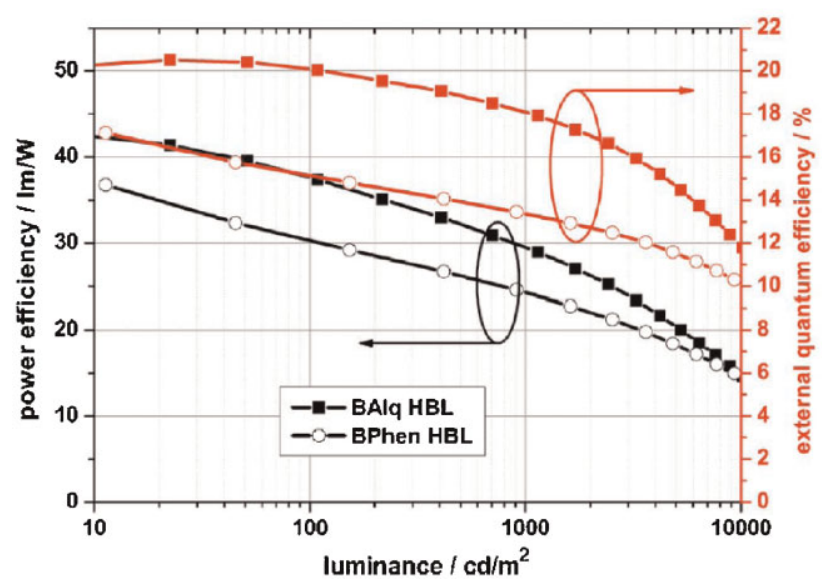

Figure 25 Design of a highly efficient orange/red OLED(top). The external quantum efficiency reaches $20 \%$ if the material BAlq is used as hole blocker. Reprinted with permission from Meerheim et al., J. Appl. Phys. 104, 14510 (2008). Copyright 2008, American Institute of Physics [101].

\subsubsection{Highly efficient monochrome devices}

4.2.1.1 Bottom emission OLEDs Monochrome OLEDs with an external quantum efficiency above $20 \%$ are well established nowadays for all monochrome colors [37, 101, 102]. The design of a highly efficient red-orange OLED is shown in Fig. 25, top. It consists of $90 \mathrm{~nm}$ ITO as anode, $50 \mathrm{~nm}$ NHT5 doped with NDP ${ }^{9}, 10 \mathrm{~nm}$ Spiro-TAD as EBL, $20 \mathrm{~nm}$ of NPB doped with the red-orange emitter $\operatorname{Ir}(\mathrm{MDQ}) 2$ (acac) (10\%), either BPhen or BALq as HBL (10 nm), BPhen doped with Cs as ETL $(60 \mathrm{~nm})$ and $\mathrm{Al}(100 \mathrm{~nm})$ as cathode [101].

The device shows the expected orange-red emission. The external quantum efficiency and power efficiency of the two samples with varying hole blocking layers are shown in Fig. 25, bottom. The OLED using BAlq is clearly superior to BPhen as blocking layer, which can be explained by a more efficient injection of electrons into the EML [101].

The device using BAlq as blocking layer reaches an external quantum efficiency of $20 \%$ at a luminance of $100 \mathrm{~cd} \mathrm{~m}^{-2}$. Considering that these results were obtained

${ }^{9}$ Novaled AG

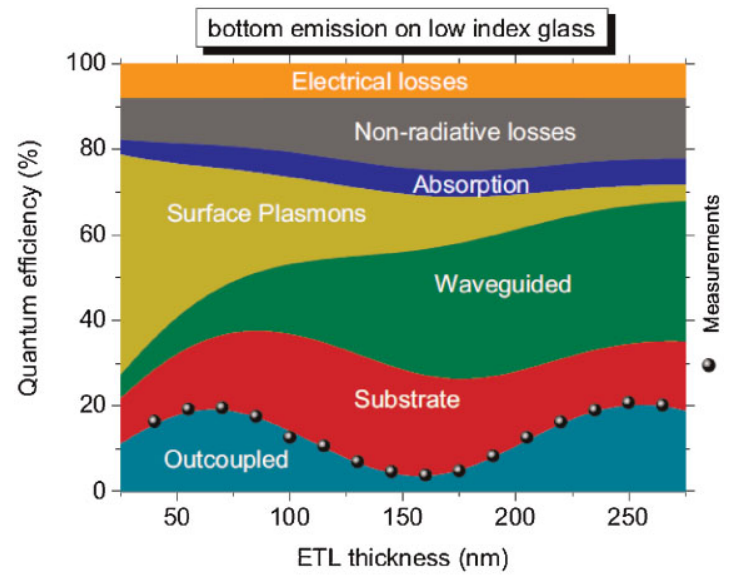

Figure 26 Influence of the OLED cavity on the external quantum efficiency of an OLED. The different loss mechanisms in a red OLED are quantified. Experimentally obtained external quantum efficiencies are shown as black dots. Reprinted with permission from Meerheim et al., Appl. Phys. Lett. 97, 253305 (2010). Copyright 2010, American Institute of Physics [95].

without the use of outcoupling enhancement methods and that only one out of five photons is coupled out of these devices, the internal quantum efficiency (IQE), i.e., the efficiency of photon generation inside the OLED, is already very high. This shows that the largest improvements in efficiency of OLEDs can be reached by an improvement of the outcoupling efficiency.

Doping is a key technology to study cavity and outcoupling effects as it allows to vary the thickness of the transport layers and to tune the cavity. The measured and calculated quantum efficiency of a series of similar orange/ red OLEDs with varying ETL thickness is shown in Fig. 26. The red OLED consisting of $60 \mathrm{~nm}$ NHT-5 doped with $4 \%$ NDP- $2^{10}$ as HTL, $10 \mathrm{~nm}$ of Spiro-TAD as EBL, $20 \mathrm{~nm}$ of NPB doped with the red phosphorescent emitter Ir(MDQ)2(acac) as EML, $10 \mathrm{~nm}$ of BAlq as HBL, and BPhen doped with Cs as ETL. The thickness of the ETL has been varied from 25 to $275 \mathrm{~nm}$ [95]. The measured external quantum efficiency for varying thickness of the electron transport layer is shown by black dots. The plot shows two distinct peaks corresponding to the first and the second resonance condition of the cavity. At these peaks the EML is positioned at the maximum of the optical field in the device, which results in an optimal outcoupling efficiency.

Apart from the measured external quantum efficiency, the different optical loss channels inside the OLED are quantified. The calculation is based on an optical model of the OLED, which treats the emitting molecules as point dipoles to calculate the radiative molecular transitions [103]. The electromagnetic field inside the OLED is calculated by a transfer matrix approach. The electrical behavior of the device is taken into account by the electrical efficiency or charge carrier balance $\gamma$.

${ }^{10}$ Novaled AG 


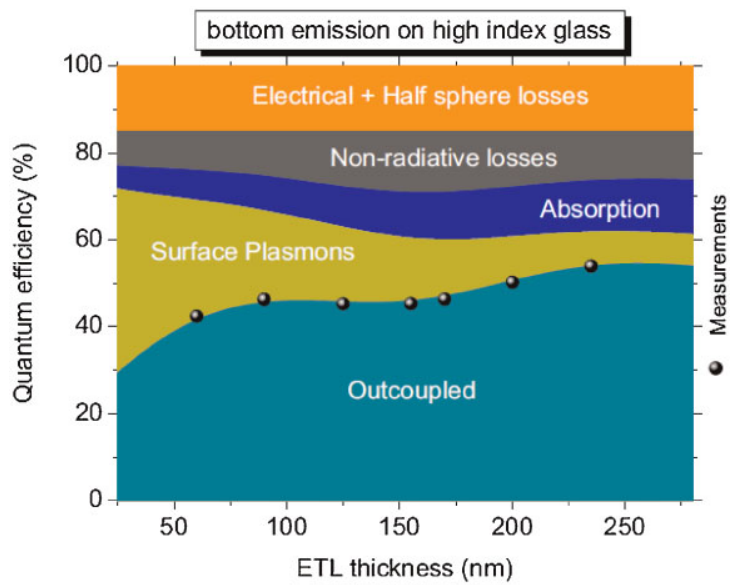

Figure 27 Simulated distribution of loss channels in a red OLED on HI glass. A half lense is used to extract all substrate modes. The measured external quantum efficiency of these devices is shown by black dots. Reprinted with permission from Meerheim et al., Appl. Phys. Lett. 97, 253305 (2010). Copyright 2010, American Institute of Physics [95].

In the OLED, energy is lost due to electrical losses (i.e., a low $\gamma$ ), non-radiative losses (i.e., a low $\eta_{\mathrm{r}}$ ), absorption in the organic layers, coupling to surface plasmons located at the metallic cathode, coupling to waveguided (organic) modes, and finally due to light that is trapped in the substrate (cf. Fig. 26). A large share of the losses in the first order devices is due to a strong coupling of the emission to surface plasmons. If the distance between emitter and cathode is increased by increasing the ETL thickness, coupling to surface plasmons decreases. However, the decrease in surface plasmons is almost completely compensated by an increase in coupling to waveguided modes, so that the overall increase in external quantum efficiency is small.

The calculations given in Fig. 26 show that a large increase in quantum efficiency is possible if all waveguided and substrate modes are extracted from the device. One method to extract these modes is the use of an index-matched glass substrate $(n=1.78)$, which avoids total reflections at the interface ITO/glass. To extract all light out of the substrate, a half lense is attached to the substrate.

The resulting external quantum efficiencies and the distribution of loss channels are shown in Fig. 27. All waveguided and substrate modes are avoided. In the second order device the external quantum efficiency is as high as $54 \%\left(104 \mathrm{~lm} \mathrm{~W}^{-1}\right)$, i.e., more than every second electron injected into the device generates a photon, which is extracted out of the device.

In summary, monochrome OLEDs with an extremely high IQE of $20 \%$ can be designed. To increase the efficiency further, the outcoupling efficiency of the OLED has to be improved. If the distance between emitter and cathode is increased by thicker ETL layers, coupling to plasmons is reduced. Furthermore, by high-index (HI) glass substrates and an index-matched halflense waveguided modes can be avoided, resulting in an external quantum efficiency beyond $50 \%$.
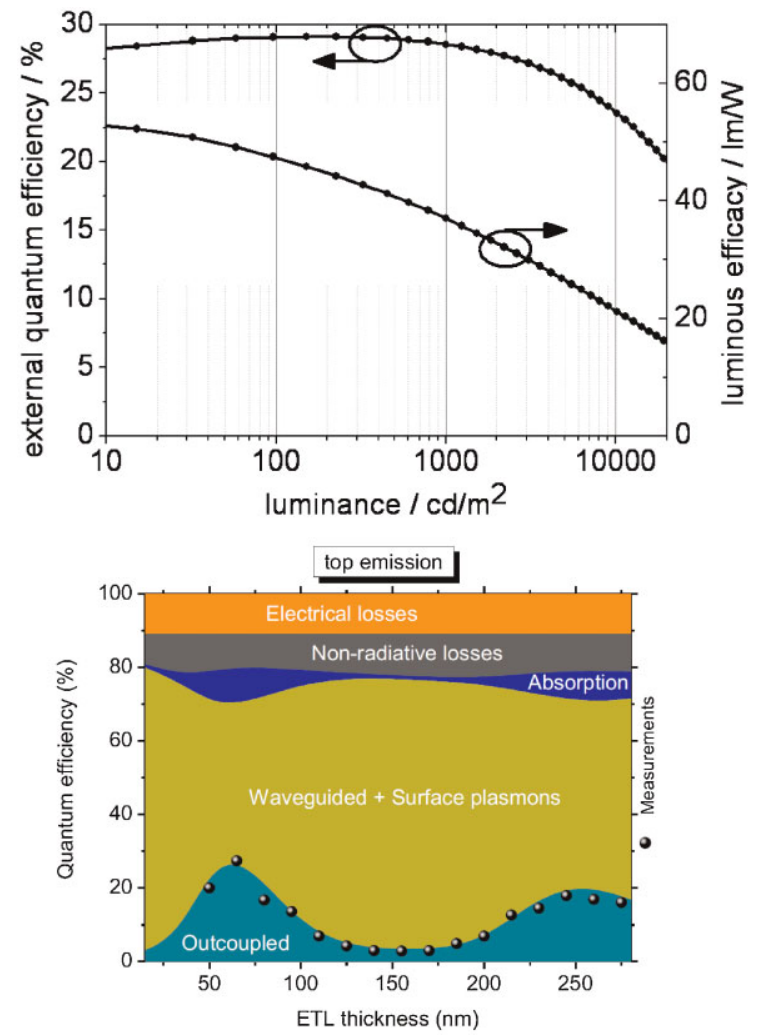

Figure 28 (Top) External quantum efficiency of a red top-emitting OLED. An external quantum efficiency of $29 \%$ is reached. Reprinted with permission from Hofmann et al., Appl. Phys. Lett. 97, 253308 (2010). Copyright 2010, American Institute of Physics [104]. (bottom) Distribution of loss channels in the top-emitting OLED. Measured external quantum efficiencies are marked by black dots. Reprinted with permission from Meerheim et al., Appl. Phys. Lett. 97, 253305 (2010). Copyright 2010, American Institute of Physics [95].

4.2.1.2 Top-emission OLEDs All OLEDs discussed so far are bottom emission devices, i.e., light is emitted through a transparent anode and a glass substrate. However, there is a great need to use low cost and flexible substrates, which would allow for a roll-to-roll production and a significant reduction in OLED cost. One alternative for thick glass substrates are flexible metal foils, which also provide a sufficient barrier to humidity and oxygen, so that high OLED lifetimes can be reached.

However, these metal substrates are opaque and light emission is only possible through a thin, semitransparent metal top electrode. These so called top-emission OLEDs show an even more enhanced microcavity, which is also observable in the calculated external quantum efficiency for varying ETL thickness as shown in Fig. 28. The device consists of $36 \mathrm{~nm} \mathrm{MeO-TPD} \mathrm{doped} \mathrm{with} 4 \% \mathrm{NDP}-2^{11}$ as HTL, $10 \mathrm{~nm}$ of NPB as EBL, $20 \mathrm{~nm}$ of NPB doped with the red phosphorescent emitter $\operatorname{Ir}(\mathrm{MDQ}) 2$ (acac) as EML, $10 \mathrm{~nm}$ of BPhen as HBL, and BPhen doped with Cs as ETL. As for

${ }^{11}$ Novaled AG 
the bottom devices, the thickness of the ETL has been varied from 25 to $275 \mathrm{~nm}$ [95].

Figure 28, top shows the external quantum efficiency and luminuous efficiency for the first order device (ETL thickness of $64 \mathrm{~nm}$ ). For these devices, a maximum external quantum efficiency of $29 \%$ is reached, which exceeds the efficiency of bottom emission devices without outcoupling enhancement [104].

The measured external quantum efficiency and the calculated loss channels for varying ETL thickness are shown in Fig. 28, bottom. In comparison to bottom emitting devices, the two peaks at the first and second order resonances are sharper, which can be explained by a stronger microcavity of top-emitting OLEDs. Furthermore, first order devices are always more efficient than second order devices, which is due to a stronger Purcell effect.

In summary, top-emission devices can be highly competitive with bottom-emitting devices, at least for monochrome devices. However, for white OLEDs, the strong cavity and the need to precisely adjust the thickness of the cavity to the emission wavelength of the emitter make the design of a broad emitting white top-OLED challenging. Thus, the discussion will be focused on bottom emitting white OLEDs in the following section.

4.2.2 p-i-n devices: White OLEDs To generate white light, the emission of several emitters has to be mixed. The most common approach to combine the emission of several emitters is to stack distinct EMLs for all colors.

Figure 29 shows the design of a highly efficient white OLED. The device is based on the $\mathrm{p}-\mathrm{i}-\mathrm{n}$ concept. It consists of an ITO anode (on HI glass, $n_{\text {high }}=1.78$ or low index (LI) glass, $n_{\text {low }}=1.51$, devices HI and LI), MeO-TPD doped with NDP-2 ${ }^{12}$ ( $4 \mathrm{~mol} \%, 60 \mathrm{~nm}$ for the OLEDs on low-n glass and $45 \mathrm{~nm}$ on high-n glass) as HTL, NPB (10 nm) as EBL, red, blue and green EMLs, TPBi $(10 \mathrm{~nm})$ as hole blocking layer, BPhen doped with Cs as ETL, and Ag as cathode. Two thickness values for the ETL layer are used (40 and $210 \mathrm{~nm}$ ) for first and second order cavities (denoted as HI-1 or LI for the first maximum and HI-2 and HI-3 for the second maximum) [40].

The EMLs are designed to ensure a distribution of excitons on all monochrome emitters (Fig. 29, lower part) leading to white emission. The EML consists of two matrix materials, TCTA, which is preferentially hole transporting and TPBi, which is preferentially electron transporting. In such a double emission layer (DEML) [37], the exciton generation zone is located at the TPBi/TCTA interface. It extends across the part of the TCTA matrix, which is doped by a red emitter $\operatorname{Ir}(\mathrm{MDQ} 2$ (acac) $(10 \mathrm{wt} \%)$ and across the FIrpic doped region of TPBi $(20 \mathrm{wt} \%)$. Thus, either red or blue excitons are generated. As the triplet energy of FIrpic is in resonance with the triplet energy of TPBi, excitons are not confined on the FIrpic molecule and can diffuse toward the green emitter $\operatorname{Ir}($ ppy)3 $(8 \mathrm{wt} \%)$. The overall distribution of excitons can be tuned by thin undoped layers between the doped layers.

${ }^{12}$ Novaled AG
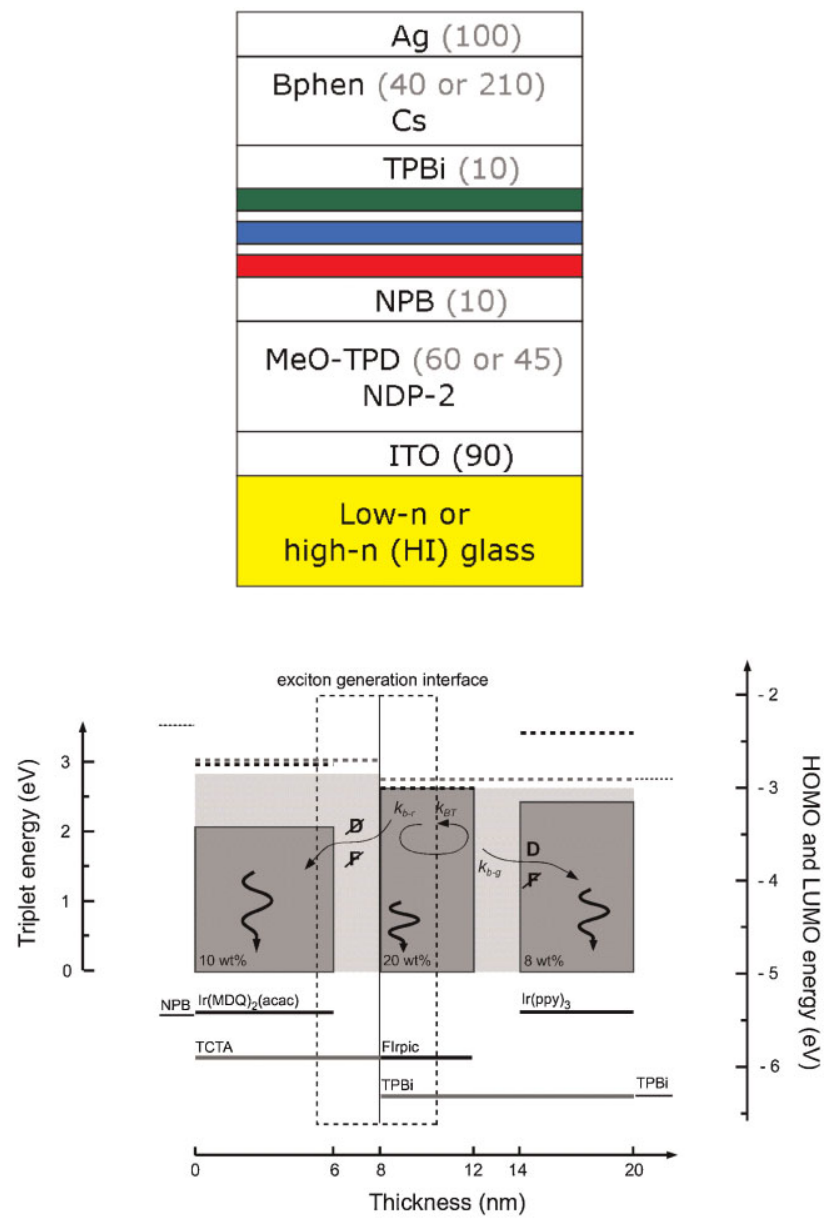

Figure 29 Highly efficient white OLED based on the $\mathrm{p}-\mathrm{i}-\mathrm{n}$ concept. Green, blue and red emission is generated in separate layers. In the lower part the design of the EML is shown $[40,105]$. Reprinted with permission from Luessem, Reineke et al., Proc. SPIE 7617, 761712 (2010).

The efficiency of these OLEDs is shown in Fig. 30. The same variations of layer structure as for the monochrome devices in Section 4.2.1 have been done, i.e., the thickness of the ETL has been varied to obtain first (LI and HI-1) and second order devices (HI-2 and HI-3). For the device HI-2 an HTL thickness of $205 \mathrm{~nm}$ was used whereas for HI-3 the HTL thickness was slightly increased to $210 \mathrm{~nm}$. Furthermore, devices were prepared on LI and on HI glass. In Fig. 30, the efficacy measured without any outcoupling enhancement is shown by continuous lines, the dotted line is measured with a structure of small pyramids cut into a sheet of HIglass applied to the substrate to enhance the outcoupling efficiency, and the dashed line is obtained by applying a half lense to the substrate.

As already shown for monochrome devices, the use of $\mathrm{HI}$ glass leads to a significant improvement in the luminous efficacy (e.g., compare the efficacy of device LI and HI-1), if either the pyramidal outcoupling structure or the halfsphere is used. 

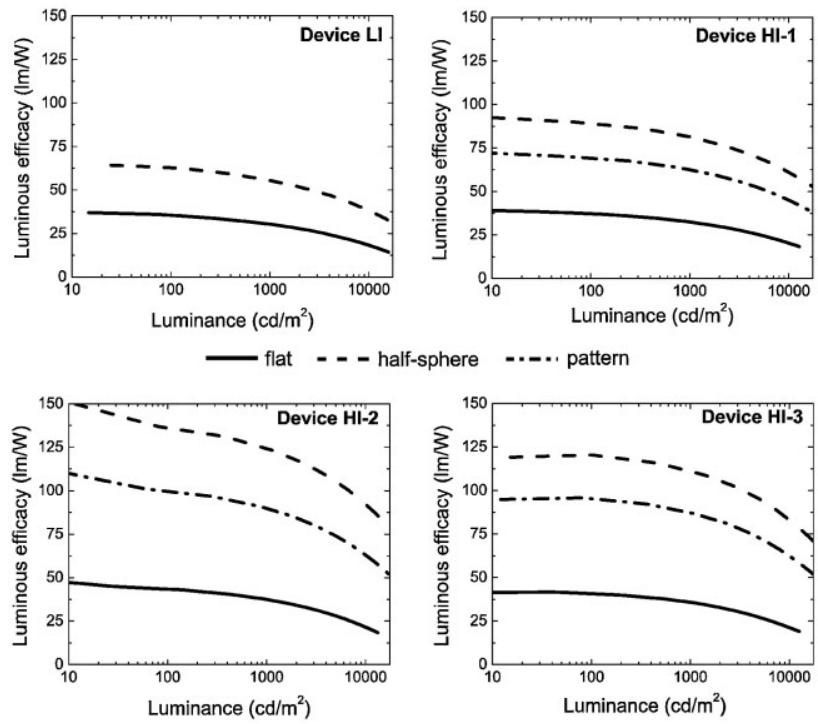

Figure 30 Efficiency of the white OLED as shown in Fig. 29. Devices are prepared on HI and LI glass. The ETL thickness has been varied to obtain OLEDs in the first (LI and HI-1) and second order (HI-2 and HI-3). The efficiency is measured without any further outcoupling enhancement methods, with a large half-lense, and with a pyramidal HI structure (pattern) [40,105]. Reprinted with permission from Luessem, Reineke et al., Proc. SPIE 7617, 761712 (2010).

Furthermore, second order devices are generally more efficient. Less emission is lost to plasmonic modes and waveguided modes are suppressed due to the use of $\mathrm{HI}$ glass. A luminous efficacy as high as $901 \mathrm{~m} \mathrm{~W}^{-1}$ at $1000 \mathrm{~cd} \mathrm{~m}^{-2}$ is obtained using the pyramidal outcoupling structure, measured in the second maximum of the cavity of the OLED structure (HI-2).

These experiments show that it is possible to very efficiently generate white light. However, one drawback of these devices is the color point $(0.41,0.49)$, which contains too less blue emission and which is off the Planckian locus. The color could be enhanced by a deep-blue phosphorescent emitter. However, even FIrpic is very instable, which leads to low lifetimes of these white OLEDs. Deep blue emitters such as FIr6 show a comparable efficiency at smaller wavelengths compared to FIrpic, but are even less stable. Hence, there is a high need to find alternatives to the all-phosphorescent approach, which avoid the use of unstable blue phosphorescent emitters, but still keep the potential of a 100\% IQE. One alternative is the triplet-harvesting approach [106-112].

4.2.3 Triplet-harvesting OLEDs In so called hybrid white OLEDs emission from a fluorescent blue emitter is mixed with emission from phosphorescent red and green emitters. Triplets generated on the fluorescent blue emitter are usually lost, as the transition to the ground state is nonradiative. However, if the triplet level of the fluorescent blue emitter exceeds the triplet level of the red and green phosphorescent emitter, triplets can be transferred to the

\begin{tabular}{|l|l|}
\hline material & thickness \\
\hline ITO & $20 \mathrm{~nm}$ \\
\hline $\begin{array}{l}\text { MeO- } \\
\text { TPD:NDP2 }\end{array}$ & $10 \mathrm{~nm}$ \\
\hline Spiro-TAD & $10 \mathrm{~nm}:$ \\
\hline 4P-NPD: & $\mathbf{8}$ wt \\
\hline Ir(MDQ) & acac \\
\hline 4P-NPD & $\mathbf{0 . . 3 0 ~ n m}$ \\
\hline BPhen & $10 \mathrm{~nm}$ \\
\hline BPhen:Cs & $30 \mathrm{~nm}$ \\
\hline Al & \\
\hline
\end{tabular}

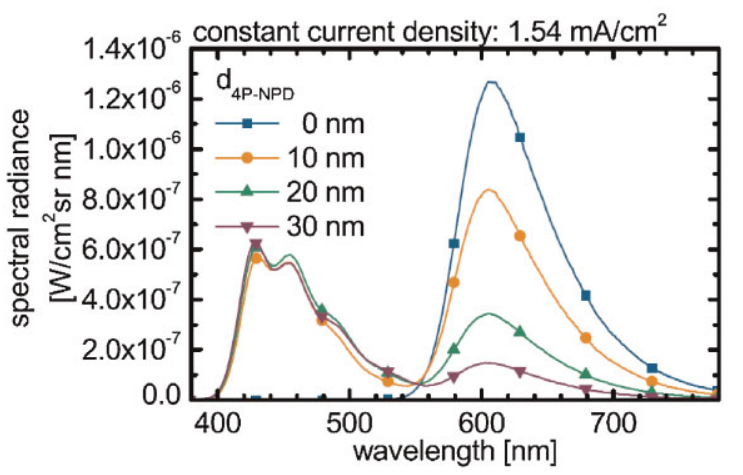

Figure 31 Triplet harvesting red/blue OLED. Excitons are generated at the interface between the hole blocking layer BPHen and the blue emitter 4P-NPD and diffuse toward the 4P-NPD layer doped with the red emitter $\operatorname{Ir}(\mathrm{MDQ}) 2$ (acac). Depending on the thickness of the 4P-NPD layer, more or less triplets reach the red emitter, which is visible in the spectrum (bottom part). As the singlet diffusion length is shorter compared to the triplet diffusion length, all singlets decay on the blue 4P-NPD layer and the blue emission does not depend on the thickness of the intrinsic 4P-NPD layer. Reprinted with permission from Rosenow et al., J. Appl. Phys. 108, 113113 (2010). Copyright 2010, American Institute of Physics [113].

phosphorescent emitter and decay radiatively. Thus, all excitons are utilized and it is possible to reach high efficiencies, although a fluorescent blue emitter is used.

A possible blue emitter is 4P-NPD [107-109]. 4P-NPD has a triplet level of $2.3 \mathrm{eV}$ [108] and allows to transfer triplets to a red emitter. The design of a red/blue triplet harvesting OLED is shown in Fig. 31, upper part [113]. As 4P-NPD preferentially conducts holes, excitons are generated at the interface between the hole blocking layer (BPhen) and the 4P-NPD layer. Starting from this interface, excitons start to diffuse toward the 4P-NPD layer doped with the red emitter $\operatorname{Ir}(\mathrm{MDQ}) 2$ (acac).

Figure 31, lower part shows the emission spectra of the device for varying thickness of the undoped 4P-NPD layer at a constant current. It can be seen that the blue emission remains constant for different 4P-NPD layer thickness, whereas the intensity of the red emission increases for 


\begin{tabular}{|l|l|}
\hline material & thickness \\
\hline ITO & 90 \\
\hline $\begin{array}{l}\text { MeO- } \\
\text { TPD:NDP2 }\end{array}$ & $40: 2 \mathrm{wt} \%$ \\
\hline Spiro-TAD & 10 \\
\hline $\begin{array}{l}\text { 4P-NPD: } \\
\text { Ir(MDQ) })_{2} \text { acac }\end{array}$ & $5:$ \\
\hline 4P-NPD & 5 \\
\hline BPhen & 10 \\
\hline BPhen:Cs & 90 \\
\hline Al & 0.5 \\
\hline $\begin{array}{l}\text { MeO- } \\
\text { TPD:NDP2 }\end{array}$ & $65: 2 \mathrm{wt} \%$ \\
\hline Spiro-TAD & 10 \\
\hline $\begin{array}{l}\text { TCTA: } \\
\text { Ir(ppy) }\end{array}$ & $10: 8 \mathrm{wt} \%$ \\
$\begin{array}{l}\text { Ir(dhfpy }{ }_{2} \text { acac } \\
\text { TPBi }\end{array}$ & $1 \mathrm{wt} \%$ \\
\hline BPhen:Cs & 10 \\
\hline Al & 75 \\
\hline
\end{tabular}
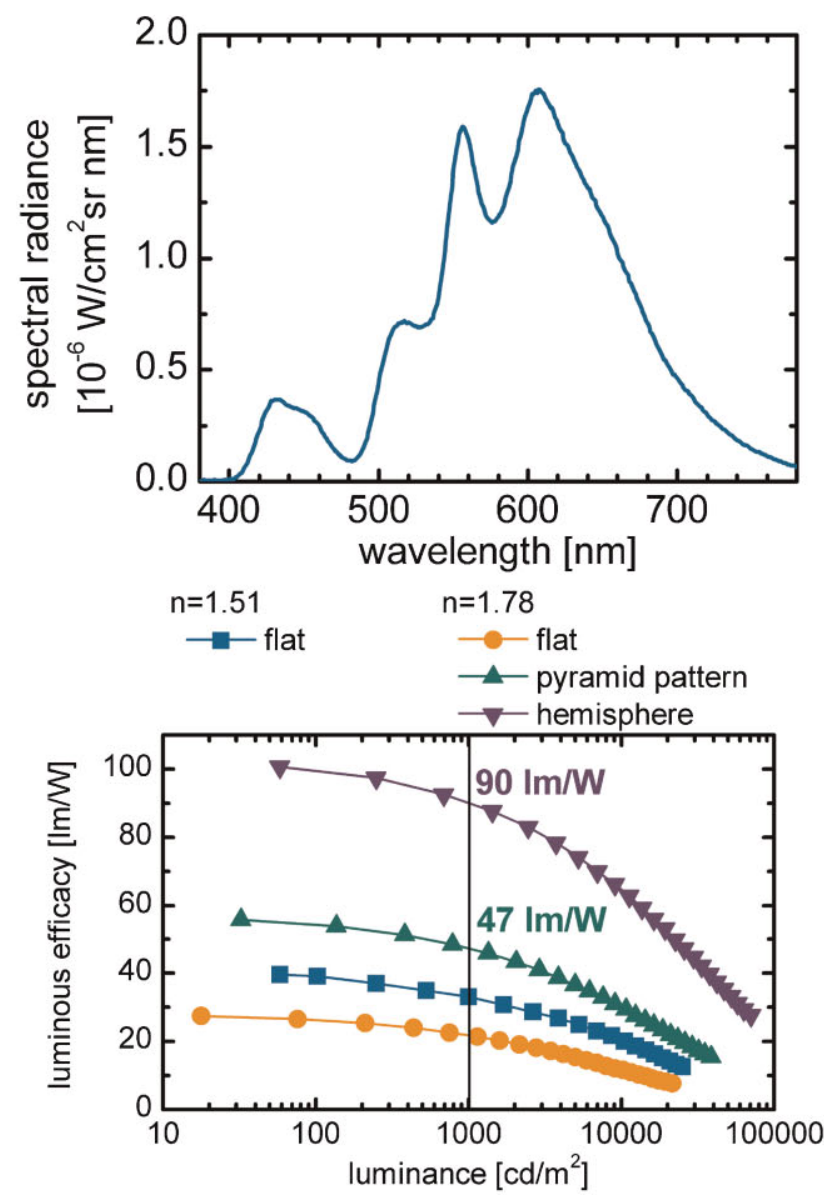

decreasing 4P-NPD layer thickness. This behavior can be understood by the different diffusion lengths of singlets and triplets. Due to their longer diffusion length, triplets can reach the doped 4P-NPD layer and recombine on the red emitter, whereas the singlets decay on the blue 4P-NPD. Therefore, depending on the thickness of the undoped 4P-NPD layer more or less triplets are harvested on the red emitter. At a thickness of $5 \mathrm{~nm}$, an optimum is reached: almost all triplets are harvested on the red emitter, but blue singlets are not quenched by the triplet emitter. A quantum efficiency of $16 \%$ is reached at $1000 \mathrm{~cd} \mathrm{~m}^{-2}$.

However, the triplet level of 4P-NPD is not sufficient to harvest triplets on green phosphorescent emitters. To fill the spectral gap in Fig. 31, a second yellow/greenish emitting OLED can be stacked onto the red/blue triplet harvesting OLED [113]. The design of such a stacked OLED is shown in Fig. 32, top. The EML of the bottom OLED consists of the matrix TCTA doped with the green emitter $\operatorname{Ir}($ ppy $) 3$ and the yellow emitter Ir(dhfpy)2(acac). The top OLED is the same OLED as shown in Fig. 31 (upper part). The two individual OLEDs are connected by a doped pn junction consisting of BPhen:Cs, a thin layer of $\mathrm{Al}$, and MeO-TPD:NDP2. In this pn junction, which is biased in reverse direction, electrons and holes are generated (cf. Section 3.2), which are injected into the upper and lower OLEDs.

The spectrum of the OLED is shown in Fig. 32 (middle). A broad spectrum with color coordinates close to the warm white point $\mathrm{A}((x, y)=(0.462,0.429), \mathrm{CRI}=80)$ is obtained. The power efficiency is presented in Fig. 32 (bottom). As for all other OLEDs discussed so far, the highest efficiency is reached for OLEDs on HI glass with a half-lense applied to the substrate $\left(90 \mathrm{~lm} \mathrm{~W}^{-1}\right)$. For the array of pyramids cut into an $\mathrm{HI}$ glass, an efficiency of $47.5 \mathrm{~lm} \mathrm{~W}^{-1}$ is reached.

4.2.4 Conclusion Doping of transport layers helps to design OLEDs with high efficiencies. The driving voltage is reduced and the thickness of the OLED cavity can be adjusted to reach a high outcoupling efficiency. Efficiencies reached by monochrome and white OLEDs are already exceeding $20 \%$ without outcoupling enhancement methods, which indicates that the IQE is impressively high. Hence, the largest improvements in efficiency can be expected by an optimization of the outcoupling efficiency $\xi$, which is currently a topic of intense research.

Figure 32 Stacked white OLED based on the triplet-harvesting concept. The device consists of a green/yellow and a red/blue triplet-harvesting unit. The two OLEDs are connected by a charge generating pn junction. In the middle part the emission spectrum of the device is shown. The spectrum is close to color point A. Using a HI hemisphere and $\mathrm{HI}$ substrates, an efficiency of $90 \mathrm{~lm} \mathrm{~W}^{-1}$ has been reached. Reprinted with permission from Rosenow et al., J. Appl. Phys. 108, 113113 (2010). Copyright 2010, American Institute of Physics [113]. 
5 Organic solar cells with doped transport layers Directly harnessing the energy from the sun will play an important role in creating a sustainable energy supply system and OSCs have the potential to become a low cost, large area photovoltaic technology in this area. Thus, they have received increasing attention in recent years and have been developed toward market introduction. Some of their main advantages are that OSC can be produced using large area coating technologies at low temperatures on flexible plastic substrates for the typically $100-200 \mathrm{~nm}$ thin organic films. Consequently, both energy and material consumption are low during production and require less than $1 \mathrm{~g}$ organic semiconductor per $\mathrm{m}^{2}$.

First experiments on the photovoltaic effect in organic semiconductors began in the 1950s with vacuum deposited small molecules [114] and the first major breakthrough was published in 1986 by Tang [10]. He introduced the concept of the planar heterojunction, i.e., using two different kinds of small molecules with dissimilar energy levels on top of each other, to facilitate the separation of the photogenerated exciton that is strongly bound. This strongly bound exciton is the main difference between OSC and standard inorganic solar cells made from, e.g., silicon where light absorption instantaneously creates free charge carriers under normal operating conditions. In organic molecules optical excitations are typically bound with energies of several $100 \mathrm{meV}$, leading to Frenkel excitons. The required electric field to separate them is more than $10^{6} \mathrm{~V} \mathrm{~cm}^{-1}$ and thus much higher that what is obtained in a working OSC. Tang's heterojunction concept provided a solution, because the energetic difference of electron donor and electron acceptor molecules at the heterojunction creates an interface for efficient exciton separation. The basic stack structure of Tang's OSC and a conceptual illustration of the heterojunction is shown in Fig. 33.

In addition to their strong binding energy, the diffusion length of photogenerated excitons is typically in the order of only $10 \mathrm{~nm}$. Thus, only excitons that are generated within their diffusion length of the heterojunction can contribute to the generation of free charge carriers. This significantly limits the photovoltaic active volume and leads to far too
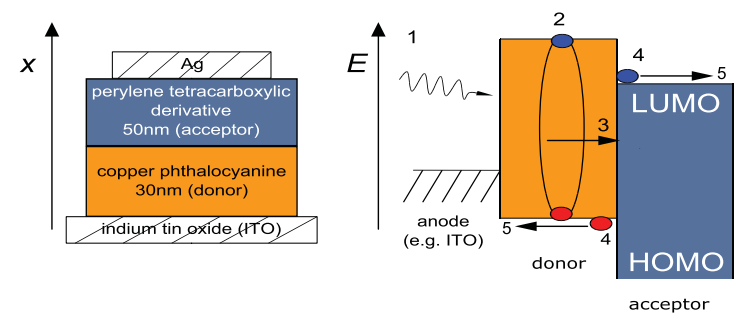

\section{7} cathode (e.g. Ag)

Figure 33 Basic layer sequence of Tang's OSC (left) and a conceptual illustration of the exciton separation at the interface between two organic materials with different energy levels (right). (1) Light exposure, (2) light absorption and exciton generation, (3) exciton diffusion, ideally to the heterojunction, (4) exciton separation, (5) charge carrier separation and extraction at opposite electrodes. little absorption for efficient light harvesting even for organic materials with absorption coefficients exceeding $10^{5} \mathrm{~cm}^{-1}$. In 1991, Hiramoto et al. presented a solution to this problem by co-evaporating donor and acceptor molecules, i.e., creating a bulk heterojunction by mixing of donor and acceptor into one layer with phase separation in the order of the exciton diffusion length [115]. This second major breakthrough showed that an efficient exciton dissociation through the complete absorbing layer possible. If suitable energy levels of donor and acceptor are chosen, this approach works very well. However, both the detailed processes and their limits for free charge carrier generation at the heterojunction as well as establishing percolation paths to the respective electrode and maintaining good transport of charge carriers in the mixed layer have remained a challenge and focus of much research both on the synthesis and processing side in recent years [116-118].

Today, all efficient OSC are based on the bulk heterojunction concept and the power conversion efficiencies starting with around $1 \%$ for Tang's device have steadily been improved since then using various strategies like better tailoring of the organic semiconductors or optimizing the stack. Current OSC efficiencies have reached more than $10 \%$ on application relevant sizes of more than $1 \mathrm{~cm}^{2}$ [119]. There are different approaches for making OSCs and they are typically categorized by their processing technology: (a) physical vapor deposition of the organic layers in vacuum, i.e., the method with which most of the initial research has been carried out [7, 76, 114, 120]; (b) processing the organic layers from solution by, e.g., spin-coating or printing, which is the process that most of current OSCs are being made with [121, 122]. In solution processing both soluble small molecules and polymers can be used as organic semiconductors. Vacuum processing limits the suitable organic semiconductors to small molecules that can be thermally evaporated without being damaged by the elevated temperatures. Since doping of organic semiconductors is most extensively being used for the latter approach, the focus will be on vacuum processing. However, in recent years the benefits of doping have lead to an increased research effort to find ways for realizing efficient doping in solution processed devices.

5.1 Solar cell characteristics Before discussing the application and benefits of doping in OSC, the standard measurements and parameters will be introduced. The main figures of merit for solar cells in general are depicted in Fig. 34. Much of the OSC research is driven by attempts to increase the power conversion efficiency $\eta$, i.e., the ratio of maximum power $P_{\text {MPP }}$ delivered by the OSC and the power of the incoming light $P_{\text {ill }}$. The extractable power is given by

$$
P_{\mathrm{MPP}}=V_{\mathrm{MPP}} I_{\mathrm{MPP}}=V_{\mathrm{oc}} I_{\mathrm{sc}} \mathrm{FF},
$$

where $V_{\text {MPP }}$ and $I_{\text {MPP }}$ being the voltage and current at the maximum power point (MPP), $V_{\mathrm{oc}}$ the open circuit voltage, $I_{\mathrm{sc}}$ the short circuit current, and FF is the fill factor. Thus, the 

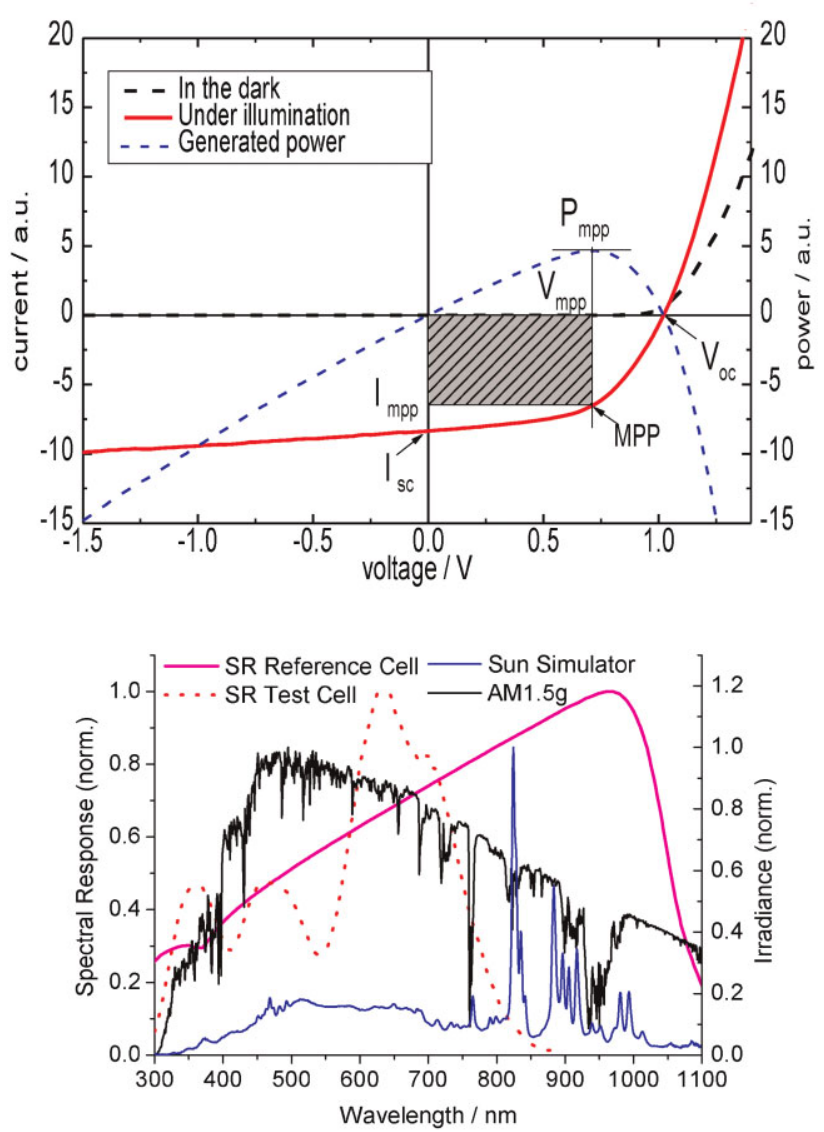

Figure 34 Top: a typical $I-V$ curve of a solar cell in the dark (black dashed) and under illumination (red) with the main parameters and the generated power (blue dashed). Bottom: a comparison between the spectral response of a typical Si reference diode (pink solid) and an OSC (red dotted) having four times fluorinated zinc phthalocyanine and $\mathrm{C}_{60}$ as photovoltaic active layer. Additionally, the differences in the reference illumination spectrum AM1.5g (black solid) and a typical solar simulator (300 W Xe high pressure lamp with AM1.5 filter, blue solid) are shown.

efficiency is defined as

$$
\eta=\frac{V_{\mathrm{MPP}} I_{\mathrm{MPP}}}{P_{\mathrm{ill}}}=\frac{V_{\mathrm{oc}} I_{\mathrm{sc}} \mathrm{FF}}{P_{\mathrm{ill}}} .
$$

As $\eta$ of a particular solar cell very much depends in the illumination spectrum, international standards have been defined for characterizing solar cells and measuring the device efficiency [123]. These should be followed for OSC, as well, but special care is necessary, e.g., because up to now no organic reference diodes are available and the spectral response of the OSC and the reference diode used to determine $P_{\text {ill }}$ differ very much [124]. Figure 34 illustrates this difference in spectral response $\operatorname{SR}(\lambda)$ that is defined as

$$
\operatorname{SR}(\lambda)=\frac{q \lambda}{h c} \operatorname{EQE}(\lambda),
$$

where $q$ is the elementary charge, $\lambda$ the wavelength, $h$ the Planck's constant, $c$ the speed of light in vacuum, and $\operatorname{EQE}(\lambda)$ is the external quantum efficiency, i.e., the ratio between extracted electron-hole pairs and incoming photons at $\lambda$ [124]. Having determined the absorption of the solar cell and the EQE, the IQE gives the ratio between extracted electron-hole pairs and absorbed photons and can be calculated as

$$
\operatorname{IQE}(\lambda)=\frac{\operatorname{EQE}(\lambda)}{\eta_{\mathrm{a}}(\lambda)},
$$

where $\eta_{\mathrm{a}}(\lambda)$ is the absorption of the solar cell. Here, either the absorption of the complete stack or only the absorption in the photovoltaic active layers can be used $[46,125]$.

Knowing the spectrum of the illumination source and the standard reference solar spectrum AM1.5g as well as the spectral response of reference diode and OSC, the efficiency can be calculated correctly using a mismatch factor for the light intensity [124, 126]. Generally, additional great care has to be taken when measuring the active area of the OSC, because the typical size for laboratory OSC is only a few $\mathrm{mm}^{2}$ which makes them prone to measurement errors [127]. For independent verification and additional certification of efficiencies, several institutions and companies around the world offer their service [119].

5.2 Organic $\mathbf{p}-\mathbf{i}-\mathbf{n}$ solar cells To further improve the efficiency, all processes from photon absorption to the extraction of charge carriers have to be better understood and optimized. Concerning the device architecture, vacuum processing and controlled doping of transport layers have several advantages. In the early days, vacuum deposition was the simplest way of making thin films of organic semiconductors, because these materials were generally hard to process. Furthermore, vacuum processing allows for an excellent control of the thickness of the deposited layers and easy fabrication of multilayer structures. This control is also key during co-evaporation of doped layers and D-A heterojunctions of two materials where fine adjustments of the composition ratio and even concentration gradients can easily be realized.

\subsubsection{Brief history of vacuum deposited organic} solar cells Before the discovery of the heterojunction concept, OSCs were basically Schottky diodes in which an organic semiconductor was sandwiched between two electrodes as shown in Fig. 35 on the left [114]. Due to the strongly bound photogenerated excitons, charge separation inside the organic layer was very ineffective, and the metal contacts were efficient exciton quenchers. Consequently, power conversion efficiencies were well below 1\%. These issues were solved by Tang and published in 1986 [10]. His bi-layer solar cells were made of a metal-phtalocyanine as electron donor and a perylene derivative as electron acceptor (see Figs. 33 and 35 second from the left). Being stacked on top of each other, the planar heterojunction offered an 


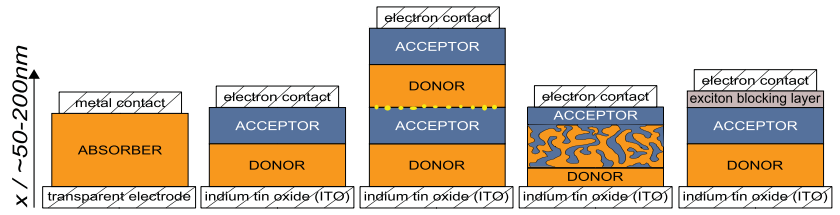

Figure 35 Illustration of various OSC stacks from left to right: a pre-Tang single layer device, Tang's planar heterojunction device concept, a tandem stack using metal clusters as recombination centers, Hiramoto's planar-bulk-planar heterojunction stack, and a stack using an exciton blocking layer.

interface for efficient exciton separation, leading to efficiencies of about $1 \%$. However, this approach still has one major drawback: due to an exciton diffusion length that is typically in the order of nanometers to a few tens of nanometers, only excitons generated within the exciton diffusion length from the heterojunction can reach the separating interface. Consequently, the effective photovoltaic active volume is very limited and despite absorption coefficients exceeding $10^{5} \mathrm{~cm}^{-1}$ only a small fraction of the incident light can be converted into free charge carriers.

Already early one, Hiramoto et al. were the first to introduce a new concept to OSC: given the limited volume of efficient exciton separation in a planar heterojunction, they adapted the concept of tandem devices, well known from inorganic solar cells, to OSC [128]. Their stack is shown in Fig. 35 in the middle. Using gold nanoparticles in the recombination zone between both subcells, they showed that this concept is feasible for OSC and can lead to improved device performances. Today, having a better understanding of the underlying device physics and a systematic tailoring new materials, tandem devices currently have the champion efficiencies of OSC [152].

However, this would not have been achieved without the bulk heterojunction concept that is often called the second breakthrough in OSCs, i.e., mixing of donor and acceptor into one layer. Already in 1991 again Hiramoto et al. coevaporated a perylene tetracarboxylic derivative and metalfree phthalocyanine into a mixed layer surrounded by pristine layers, thus publishing the first bulk heterojunction, the stack of which is shown in Fig. 35, second from the right $[115]^{13}$. In this general concept, the phase separation between donor and acceptor can be made to be in the order of the exciton diffusion length. Thus, excitons can be separated throughout the complete layer. However, the challenge is to establish percolation paths to the respective electrode and maintain good transport of charge carriers through the mixed layer. This challenge still remains today.

Efficiencies remained at about $1 \%$, but starting from about 2000 onwards, significant progress in the under-

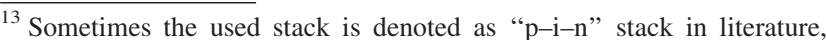
because it consists of a pristine donor layer/mixed donor:acceptor layer/ pristine acceptor layer. However, it does not use deliberately doped layers and thus is not a $\mathrm{p}-\mathrm{i}-\mathrm{n}$ stack in the general sense.
}

standing of OSCs and improvements in device efficiency were made. The number of available organic semiconductors has considerably grown since then, stacks haven been optimized and various strategies followed to investigate the OSC fundamentals and to create a viable product.

In 2000, Rostalski and Meissner used the fullerene $\mathrm{C}_{60}$ as electron acceptor in a bulk heterojunction with a stack similar to the one used by Hiramoto et al. in 1990 [130]. Fullerenes have very strong electron accepting character and had been shown to be an excellent acceptor material in solution processed OSCs with polymers as donor [131]. Up to now they have remained the main choice of materials for the acceptor due to its unique properties.

Progress in the stack design was shown by Peumans et al. in 2003 who introduced a dedicated thin exciton blocking layer of bathocuproine (BCP) between the acceptor layer and the cathode. This is illustrated in Fig. 35 on the right. The $\mathrm{BCP}$ layer is efficiently blocking excitons and preventing damage to the active layer during metal deposition and leading to power conversion efficiencies of about 3.6\% [7]. With rising efficiencies, blocking excitons from being quenched at the contacts has become a very relevant step.

An important milestone in the development of efficient OSC was finding an efficient way to tune the morphology of the bulk heterojunction. This technique was first discovered for solution processed OSC $[132,133]$ and later adapted for vacuum processed solar cells $[134,135]$. The method for extrinsically tuning the morphology is heating the substrate during the film formation, allowing molecules to better diffuse and rearrange on the surface. Overall, this has become a key component in realizing the most efficient OSC in vacuum.

The concept of deliberate molecular doping of organic materials, the main focus of this section, has also found its way in OSCs. Pfeiffer et al. used F4-TCQN (see Fig. 3) as strong electron acceptor for p-doping [41]. Doping on the p-side was later complemented with doping on the n-side, leading to the $\mathrm{p}-\mathrm{i}-\mathrm{n}$ concept for OSC, that uses both $\mathrm{p}$ - and n-doped layers surrounding the photovoltaic active layer [75]. The advantages of this concept will be the focus of the remaining part of this section.

5.2.2 Advantages of molecular doping in OSC The doping fundamentals were already described in Section 2 and doped wide-gap transport layers can extensively be used with similar advantages, i.e., control of Fermi levels, quasi-Ohmic contacts to electrodes, for OSC as for OLEDs (see Section 2).

Tang's solar cell of 1986 had a planar heterojunction for efficient exciton separation. However, given the strongly bound nature of the photogenerated excitons and their short diffusion length, most of the excitons in the absorbing volume could not reach the heterojunction and contribute to the photocurrent. This is illustrated in Fig. 36 on the left side. Reducing the layer thickness to the photovoltaic active volume generally does not work because of short circuits and recombination at the organic-metal interfaces. Thus, the 

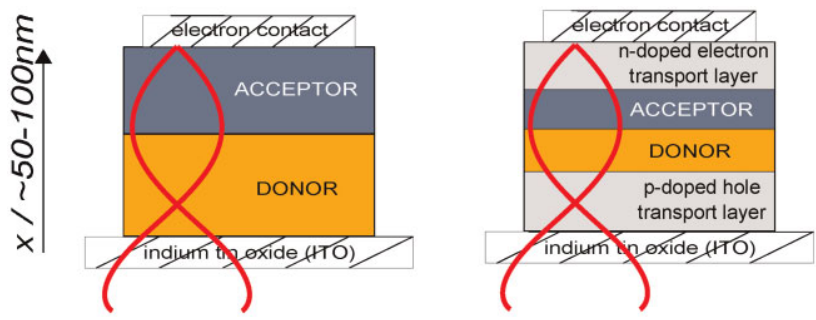

Figure 36 Comparison of the Tang's solar cell (1986) and a p-i-n solar cell structure with doped wide-gap transport materials (2004), including a schematic illustration of the optical interference pattern.

parasitic absorption in the volume that cannot contribute to the photocurrent is a severe limitation.

Using doped wide-gap transport layers, the donoracceptor layer thickness can be reduced to the photovoltaic active volume without risking shorts and recombination at the organic-metal interfaces. The corresponding layer structure for a typical $\mathrm{p}-\mathrm{i}-\mathrm{n}$ stack is illustrated in Fig. 36 on the right-hand side. It consists of a transparent hole contact, a p-doped wide-gap HTL, a photovoltaic active layer, a n-doped wide-gap ETL, and an electron contact. This stack is not only advantageous for planar heterojunctions, but is used also for bulk heterojunctions or a combination of both.

By replacing the absorbing, but not photovoltaic active volume of Tang's solar cell with wide-gap materials, the parasitic absorption is significantly reduced. Ideally, light absorption then only takes place in the photovoltaic active layers that are positioned such that photon absorption and IQE is maximized. If the wide-gap materials are doped, as described in Section 2, the layers can reach conductivities of more than $10^{-5} \mathrm{~S} \mathrm{~cm}^{-1}$, i.e., values with allow an extension of the layer thickness to $100 \mathrm{~nm}$ and more without introducing noticable Ohmic losses. Consequently, such layers not only serve as efficient charge transport layers, but can furthermore be used as optical spacers of an optical device optimization. Secondly, doping commonly ensures and Ohmic contact between electrodes and organic layers [14] and e.g. ITO can be used as either hole or electron contact [46]. Lastly, by choosing suitable wide-gap materials the contacts can be made selective: excitons are blocked from reaching the typically quenching electrode interface and charge carriers can only reach their contact, ideally without a barrier or a loss in free energy. The energy level diagram of a $\mathrm{p}-\mathrm{i}-\mathrm{n}$ solar cell structure is shown in Fig. 37 and is close to the generic and ideal solar cell structure proposed by Würfel [136].

Overall, the $\mathrm{p}-\mathrm{i}-\mathrm{n}$ concepts allows for many degrees of freedom in optimizing the absorption of the active layers, choosing the contact materials, as well as the order of the layers. In recent years, the $\mathrm{p}-\mathrm{i}-\mathrm{n}$ concept has been proven to be a versatile concept for both investigation of the fundamental processes in OSCs as well as a solid base for device optimization. In the meantime, other OSC approaches, particular those based on solution processing,

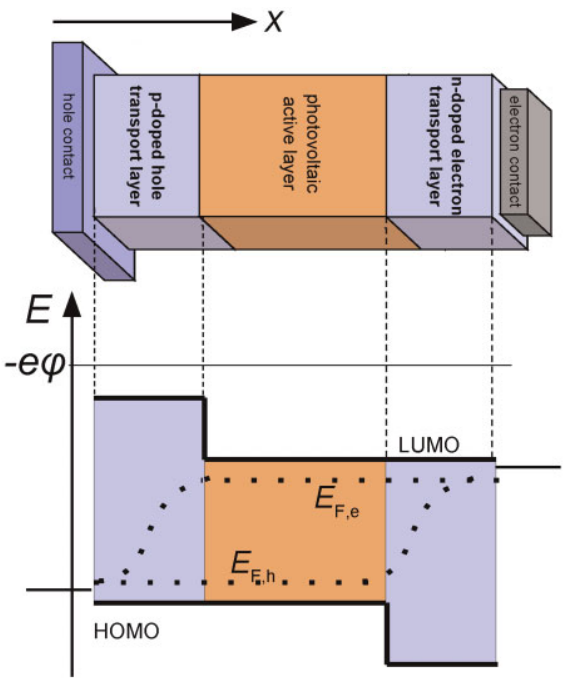

Figure 37 The stack of $\mathrm{p}-\mathrm{i}-\mathrm{n}$-OSC schematically shown in spatial coordinates (top) and a simple energy diagram (bottom). The photovoltaic active layer can be a planar heterojunction, a bulk heterojunction, or a combination of both. Both for ETL and HTL wide-gap materials are used, leading to an energetic offset to the photovoltaic active layer.

also started looking closer into doping of organic materials, thus trying to realized a full $\mathrm{p}-\mathrm{i}-\mathrm{n}$ concept.

5.2.3 Optical optimization There are various ways of optimizing OSC, and one important way is putting the absorbing layers where they can absorb most. For nontransparent OSC the reflecting metal electrode creates a strong interference pattern with a node at the organic-metal interface. Thus, the light intensity close to the metal contact is low. Since the useful thickness of the absorbing layers is limited either by the exciton diffusion length in the case of a planar heterojunction of charge carrier transport to the electrodes in the case of a bulk heterojunction, placing the photovoltaic active layer close to the metal contact will limit the achievable photocurrent. Often, a thin transparent buffer layer of BCP or bathophenanthroline (BPhen) is used to shift the absorbing layers closer to the maximum of the optical interference pattern [44, 137]. Although these layers have advantages over putting the metal contact directly into contact with the absorbing heterojunction, the thickness of this kind of buffer layer is typically limited to $\leq 15 \mathrm{~nm}$ due to its low conductivity and charge carrier mobility [7]. Here, doped transport layers again have significant advantages, because doping leads to a high conductivity allowing for spacer layer thicknesses of more than $100 \mathrm{~nm}$ without causing significant transport losses.

Figure 38 shows a p-i-n stack where the thickness $d_{\text {ETL }}$ of the n-doped ETL, here using 2,3,8,9,14,15-hexachloro$5,6,11,12,17,18$-hexaazatrinaphthylene $\left(\mathrm{HATNA}-\mathrm{Cl}_{6}\right)$, is varied between 0 and $140 \mathrm{~nm}$ [138]. HATNA-Cl ${ }_{6}$ has an optical gap of $2.7 \mathrm{eV}$, i.e., it is mostly transparent in the visible region of the sun spectrum, and an EA) of $4.07 \mathrm{eV}$ 


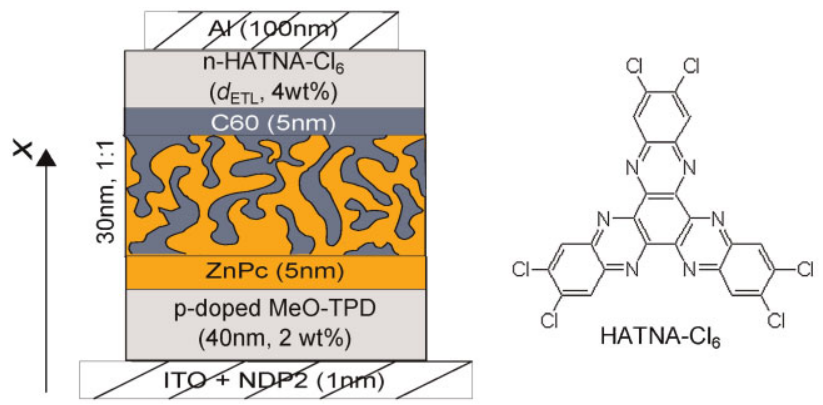

Figure 38 Simple test stack to illustrate the advantages of doped transport layers for optical optimization of the stack. The stack uses a combination of a planar and bulk heterojunction (1:1 volume ratio $\mathrm{ZnPc}: \mathrm{C}_{60}$ ) as photovoltaic active volume. The structure of the electron transport material, 2,3,8,9,14,15-hexachloro5,6,11,12,17,18-hexaazatrinaphthylene (HATNA-Cl ${ }_{6}$ ) is shown on the right. $\mathrm{p}$ - and n-doping are realized using NDP2 and NDN1 (both Novaled AG), respectively. $d_{\text {ETL }}$ of the n-doped HATNA-Cl 6 is systematically varied between 0 and $140 \mathrm{~nm}$ to move the photovoltaic active volume with respect to the $\mathrm{Al}$ mirror and thus into the interference pattern forming in the stack under illumination.

[139]. Thus value matches closely the EA of $\mathrm{C}_{60}(4.0 \mathrm{eV}$ [140]), the standard electron acceptor, and therefore allows for a barrier free extraction of electrons from $\mathrm{C}_{60}$. Doping HATNA-Cl ${ }_{6}$ with a few wt $\%$ of NDN1, a commercial dopant by Novaled AG, leads to a conductivity in the order of $10^{-3} \mathrm{~S} \mathrm{~cm}^{-1}$, a value high enough for realizing even thick ETLs.

Optical simulations were carried out using a numerical algorithm based on the transfer matrix formalism and Poynting vector calculation [141, 142]. The optical constants of the organic materials were determined using transmission and reflection measurements [143], but other methods like ellipsometry could be used as well. From the calculated absorption in each photovoltaic active layer (here $\mathrm{ZnPc} /$ $\left.\mathrm{ZnPc}: \mathrm{C}_{60} / \mathrm{C}_{60}\right)$, it is possible to calculate the maximum possible current that can be extracted from the solar cell by assuming an IQE of unity. Figure 39 shows the comparison of $j_{\mathrm{sc}}$ determined in experiments and simulations [138]. As the solar cells were measured with a solar simulator, its illumination spectrum was used as input for the optical simulation for a better comparability to the measurements and as the optical simulations are based on a series of assumptions, like e.g. IQE $=1$ at $0 \mathrm{~V}$ the absolute values of $j_{\mathrm{sc}}$ will be different, most likely lower.

Depending on where the photovoltaic active layers are positioned, a variation in $j_{\mathrm{sc}}$ of a factor of more than four is observed. This is a direct result of shifting the absorbing layers with respect to the optical interference pattern. The optimum $j_{\mathrm{sc}}$ is found at $d_{\mathrm{ETL}}$ of $50 \mathrm{~nm}$. Increasing $d_{\mathrm{ETL}}$ to about $140 \mathrm{~nm}$ puts the absorbing layer into the first interference minimum and thus much fewer photons can be absorbed. The only small variations in FF between maximum and minimum illustrate the good transport through the doped ETL. $V_{\mathrm{oc}}$ varies slightly due to changes in the splitting of the
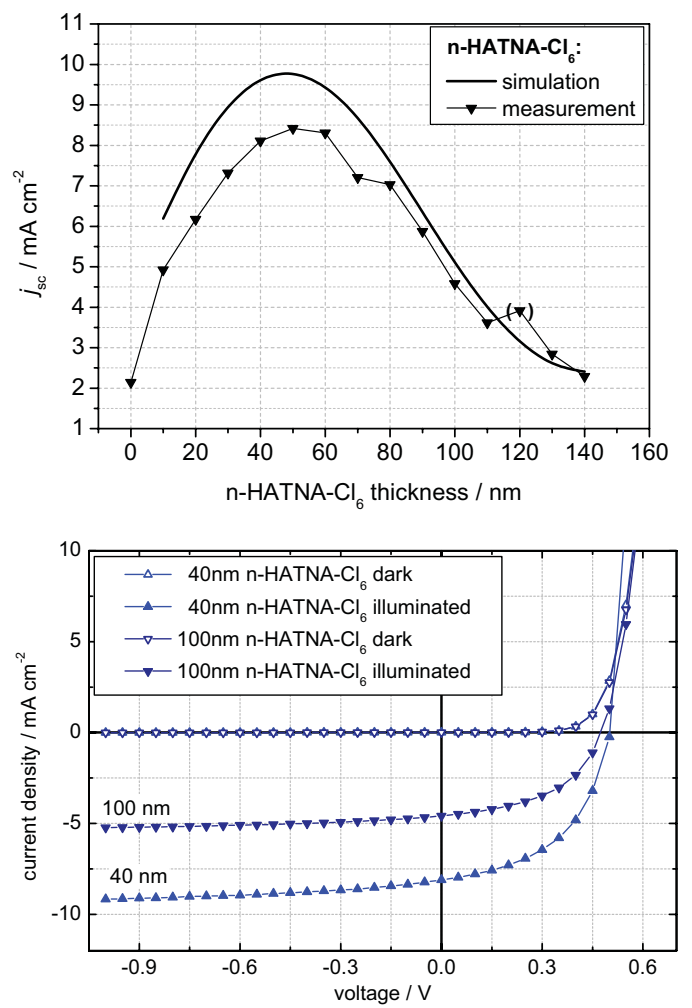

Figure 39 Short circuit current density $j_{\mathrm{sc}}$ from experiment and optical simulations assuming an IQE of unity using the sun simulator spectrum for varying thicknesses $d_{\text {ETL }}$ of the n-doped transport layer HATNA-Cl $l_{6}$ (top) and selected $I-V$ curves to demonstrate the photovoltaic performance (bottom). The value at $120 \mathrm{~nm}$ is the only outlier in the $j_{\mathrm{sc}}$ measurements. The corresponding efficiencies for 40 and $100 \mathrm{~nm}$ n-HATNA-Cl $\mathrm{C}_{6}$ are 2.1 and $1.1 \%$.

quasi-Fermi levels, because the absorption in the photovoltaic active layers changes with varying $d_{\mathrm{ETL}}$. Consequently $\eta$ is mainly determined by the variations in $j_{\mathrm{sc}}$.

These results show that doped transparent transport layers, here in the example of n-doped HATNA-Cl $\mathrm{H}_{6}$, are a key for optical optimization of OSC. This principle not only applies to vacuum processed OSC, but to all kind of OSC as long as well reflecting electrodes are used and the film thickness is below the coherence length of the sun light. Also for semi-transparent OSC, the optical optimization and the use of window layers is crucial for achieving efficient devices [144]. However, the effects of thin film optics are generally less dominant in solution processed OSC, because the thickness of the absorbing layer often exceeds $200 \mathrm{~nm}$.

5.2.4 Tandem devices Certified single heterojunction OSC efficiencies of above $8 \%$ have been reported in press releases and literature [119, 145, 146, 152]. Still under discussion is how high the efficiencies of single heterojunction devices can be pushed, but estimates based on experimental considerations put the limit to between 10 and $11 \%$, depending on the assumptions [147, 148]. Although $10 \%$ for a single heterojunction solar cell has recently been 


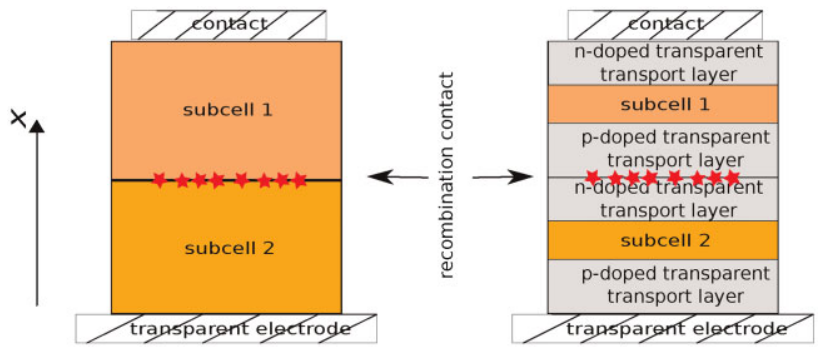

Figure 40 Stack of a generic two-terminal tandem stack consisting of two subcells connected by a recombination contact and a $\mathrm{p}-\mathrm{i}-\mathrm{n}$ tandem stack.

achieved by Mitsubishi Chemical [119], these values are just about the supposedly required values for large scale applications. Consequently, tandem concepts involving the stacking of single junction solar cells as shown in Fig. 40 are used to exceed the $10-11 \%$ limit. Their efficiencies are estimated to potentially reach about $15 \%$ $[149,150]$, because transmission and thermalization losses, two major loss mechanisms, can in principle be reduced. Thus much research has been devoted in recent years in realizing tandem OSC [151] which are currently leading the device efficiencies both for solution processed and vacuum processed OSC [119, 152]. Here, the ease and control of multilayer deposition in vacuum as well as the doping prove to be big advantages of this approach. In addition to complementary absorbers, efficient tandem devices require a well working and versatile recombination contact and current matching between the subcells, both of which can be well realized based on the $\mathrm{p}-\mathrm{i}-\mathrm{n}$ single concept as shown in Fig. 40. There are various concepts for tandem devices [151] and in the following the focus will be on the two-terminal design.

5.2.4.1 Recombination contact One central component of any two-terminal tandem is a versatile recombination contact that leads to a recombination of electrons from one subcell and holes from the other subcell without loss in free energy at all applied voltages. Hiramoto et al. showed already in the first OSC tandem publication in 1990 that metal clusters can be used as recombination centers between donor and acceptor. This approach is still used in many vacuum deposited tandem OSC, because it can prevent the splitting of the quasi-Fermi levels at this interface between the subcells. However, it has some drawbacks, as it is not a generic solution and thus does not work for any material combination of donor and acceptor. Additionally, optical optimization would be difficult, because donor and acceptor are directly next to each other. Other approaches, like the use of absorbing molecules that have the right energy levels for the recombination contact [153] or the incorporation of metal oxide interlayers $[154,155]$ have similar drawbacks.

Here, doping of organic layers was found to be very beneficial, as well, as evaporating highly doped ETL and HTL on top of each other allows for the creation of a well working recombination contact [156, 157]. This approach has the advantage that it leads to a complete addition of the open circuit voltages of the subcells in most cases and a high FF in tandem devices. By shifting the recombination contact into transport layers it can be used independently of the absorber materials, i.e., it provides a generic solution for the recombination contact. Furthermore, the use of wide-gap materials ensures high transparency, separates the recombination areas of charge carriers and excitons, and can be created using the same processes as for the rest of the tandem stack. Figure 41 shows the difference between using undoped layers, using gold clusters, and using doped transport layers as recombination contact in a test stack.

The very simple stack shown in Fig. 3 uses MeO-TPD for both the p-doped transparent HTL and electron donor. $\mathrm{C}_{60}$ is used as electron acceptor and n-doped ETL. All three shown tandem devices have similar current in reverse direction, indicating that they generate the same amount of free charge carriers. The $I-V$ characteristics show that without doped or Au nanoclusters the forward current is limited because of the backward directed heterojunction between the undoped MeO-TPD and $\mathrm{C}_{60}$. The $V_{\text {oc }}$ of the tandem OSC reaches only a value close to that of the single heterojunction. Inserting $1 \mathrm{~nm}$ Au between the subcells introduces metal clusters that act as efficient recombination centers leading to nearly twice the $V_{\mathrm{oc}}$ of the single cell. By inserting highly doped transport
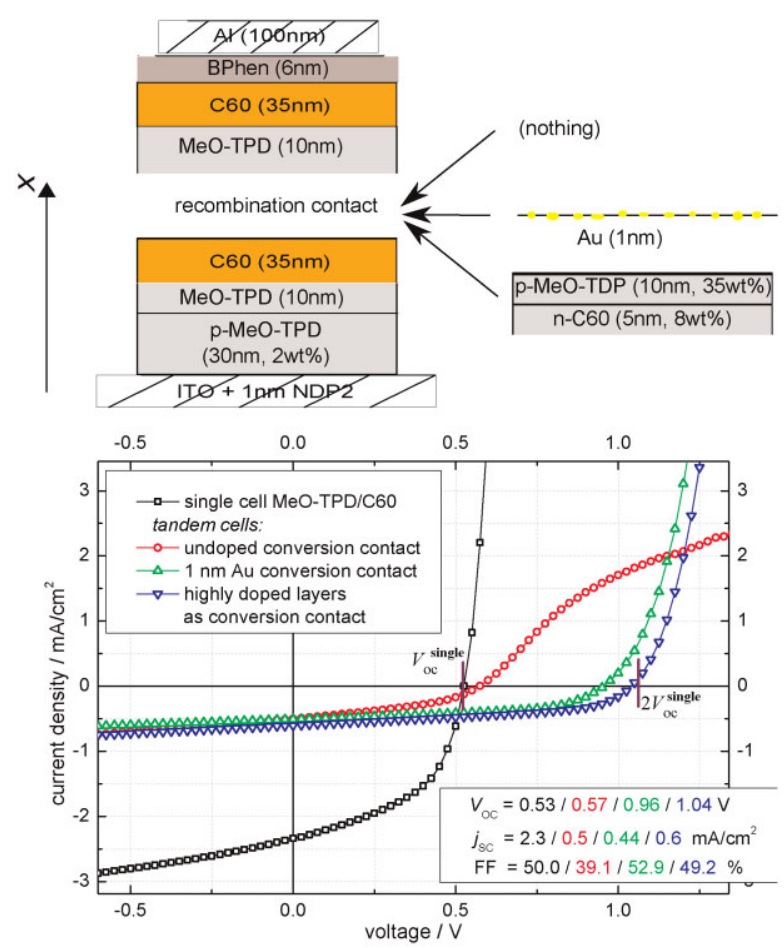

Figure 41 A simple test stack for investigating the recombination contact based on doped transport layers (top) and the corresponding results (bottom). A recombination contact either no interlayer, $1 \mathrm{~nm}$ $\mathrm{Au}$ or highly doped transport layers are inserted. The transport layers are doped at the given percentage (n-doping: AOB, p-doping: F4-TCNQ). 
layers for the recombination contact an addition of the $V_{\mathrm{oc}} \mathrm{s}$ of the subcells is reached retaining also a good FF. The general applicability of this approach has further been tested with transport materials of various energy levels, where it was found that thin interlayers of $\mathrm{Au}$ or $\mathrm{Ag}$ do not work well [156]. Due to its versatility, this kind of recombination contact is used in most efficient $\mathrm{p}-\mathrm{i}-\mathrm{n}$ tandem OSC [158, 159].

The reason for the differences between the three recombination contacts was investigated using UPS for the material combination MeO-TPD and $\mathrm{C}_{60}$ as displayed in Fig. 41. The results show that metal nanoclusters or doped transport layers are significantly different. In comparison to an interface between the two subcells without modification, both approaches reduce the tunneling distance between donor HOMO and acceptor LUMO. However, while metal clusters lead to an accumulation of charge carriers, the high doping of the layers in the recombination zone allows for a tunneling of charge carriers through a thin depletion layer [157].

5.2.4.2 Complementary absorption Whereas the first tandem devices used the same absorbing molecules in both subcells [128], the number of available materials has grown considerably. Figure 42 shows only a small selection of light absorbing molecules and their corresponding extinction coefficients. This figure also illustrates why organic materials are particularly interesting for tandem concepts: the used organic semiconductors have typical absorption coefficients above $10^{5} \mathrm{~cm}^{-1}$ at their absorption maximum and can be tailored to have well defined absorption bands. Thus, the thickness limit of the light absorbing layers due to excitonic effects, low charge carrier mobilities and lifetime can be circumvented by stacking single heterojunction solar cells and by choosing subcells with complementary absorption. Hence, a large spectral coverage of the solar spectrum with efficient absorption can be realized. However, efficiently harvesting the NIR is still a challenge.

5.2.4.3 Current matching Given that the current through a two-terminal tandem device is limited by the current of the weaker subcell, optimization of absorption and current generation in the subcells is key for efficient tandem solar cells independent of the used semiconductors. Although tandem OSC are thicker than single heterojunction OSC their absorption is still subjected to interference and microcavity effects. Taking these effects into account is crucial.

Yakimov and Forrest who used only Ag nanoclusters in their tandem devices found that the efficiency increased from one to two stacked heterojunction, but it did not increase when adding more than two subcells [162]. While they were able to fit two heterojunctions into the first interference maximum, because the organic layers were thin enough, some of the following subcells had to be in an optical interference minimum due to missing optical spacers and thus were limiting the current of the tandem devices.
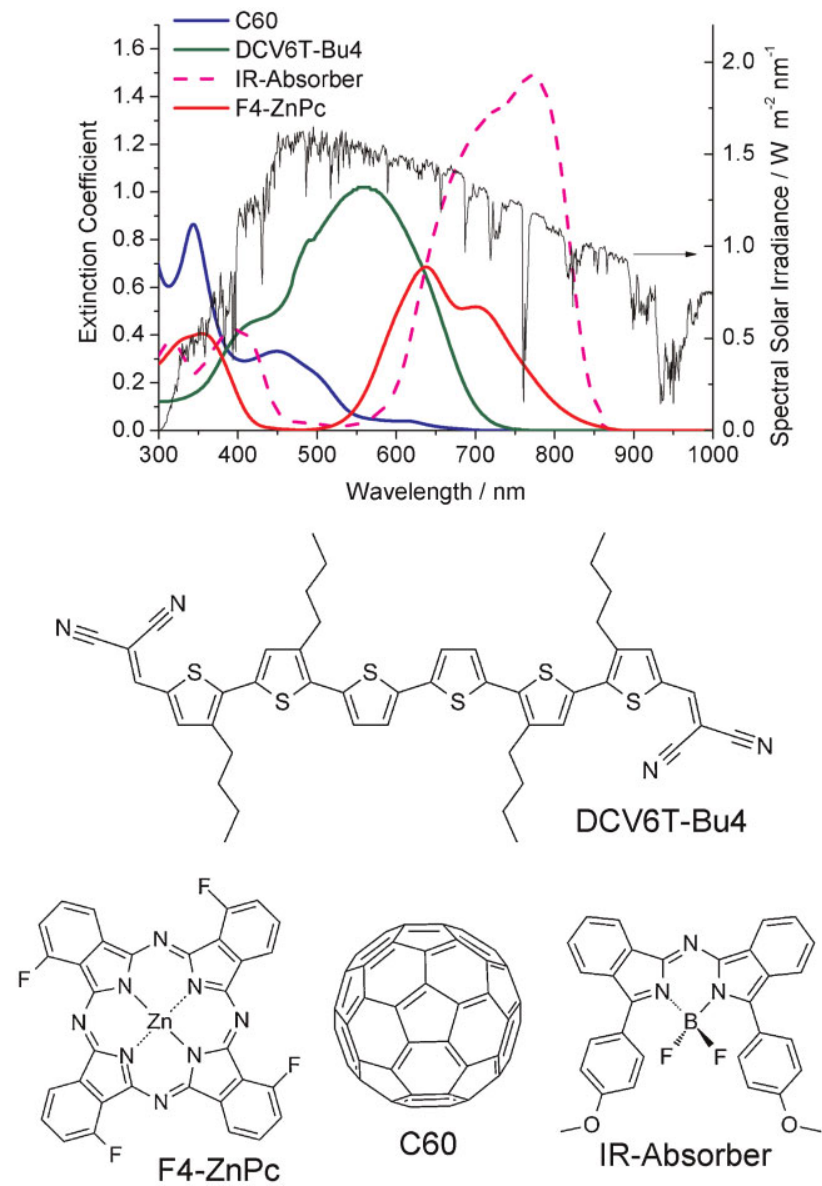

Figure 42 Extinction coefficients of some selected small molecules versus the solar spectrum. Shown are the values for $\mathrm{C}_{60}$, a dicyanovinyl substituted sexithiophene with four butyl side chains (DCV6T-Bu4, Heliatek GmbH) [46], a four times fluorinated zinc phthalocyanine (F4-ZnPc, BASF SE) [160], and an NIR absorber based on AZA-bodipys [161].

Stacking $\mathrm{p}-\mathrm{i}-\mathrm{n}$ OSC into tandem devices allows for a simple way of using the interference pattern as advantage, because the doped transport layers can be used as optical spacer between the absorbing layers of the subcells. If the subcells are thin and can be positioned in the first interference maximum $(\leq 100 \mathrm{~nm})$, usually no optical spacer between the subcells is required [158]. However, despite the high absorption coefficients of the organic absorbers, the tandem OSC would be optically thin. Once the absorbing layers of the subcells become to thick to not fit best into the first interference maximum any more, an optical spacer is required. If both subcells can be made thick enough to each cover their complete interference maximum. The former condition is currently the case for the best vacuum processed OSC and much work is devoted to further increase the thickness of the absorption layers.

Figure 43 shows the stack of a recently published twoterminal tandem OSC based on the $\mathrm{p}-\mathrm{i}-\mathrm{n}$ concept [159]. The stack consists of two subcells with nearly complementary 

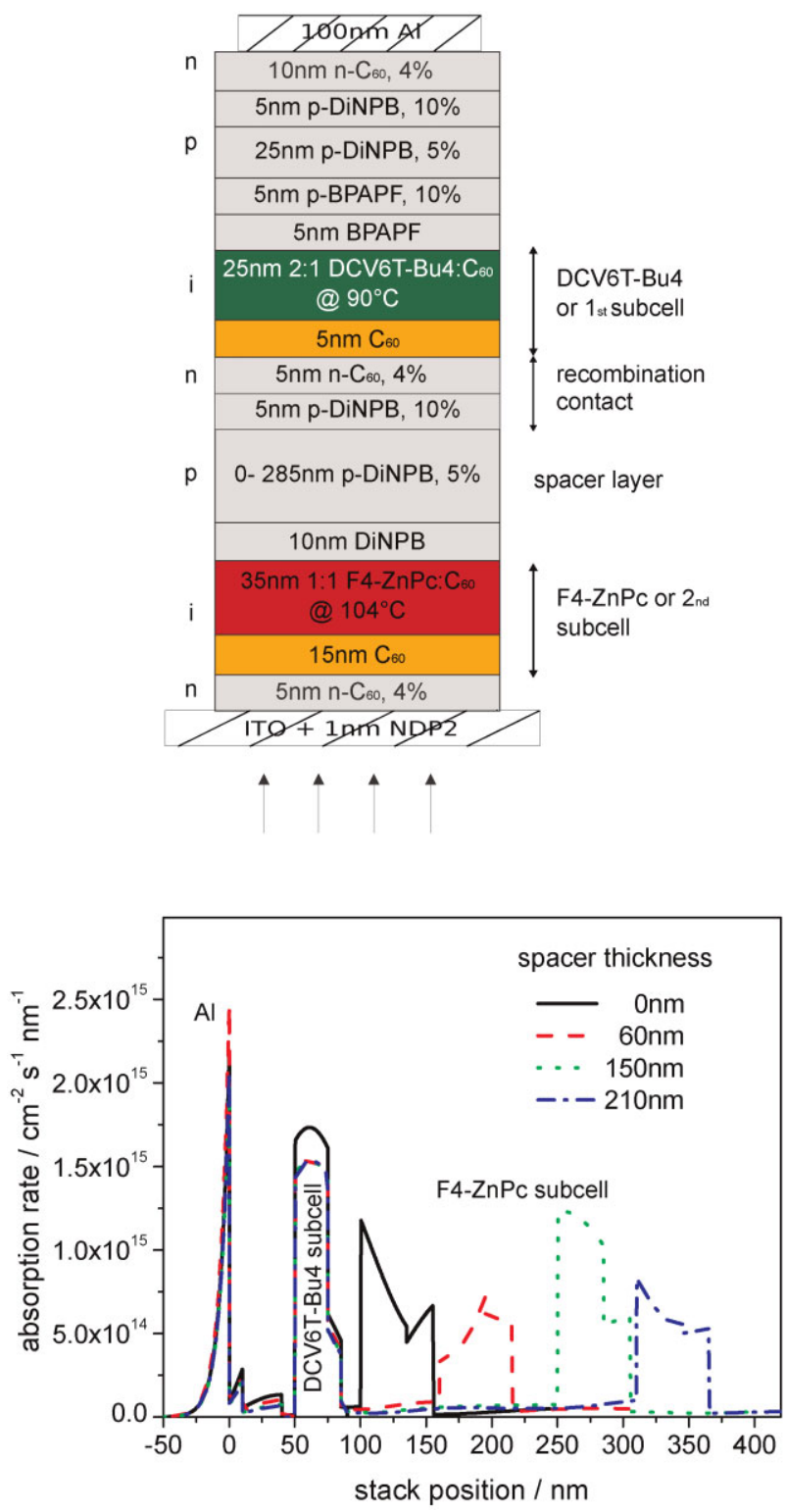

Figure 43 The stack structure of the tandem device used for the spacer variations, based on two inverted $\mathrm{p}-\mathrm{i}-\mathrm{n}$ OSC with complementary absorbing subcells of F4-ZnPc: $\mathrm{C}_{60}$ and DCV6T-Bu4: $\mathrm{C}_{60}$ (top) and the results of the optical simulation (bottom) that shows the absorption rate as function for four different spacer layer thicknesses.

absorption (F4-ZnPc: $\mathrm{C}_{60}$ and DCV6T-Bu4: $\mathrm{C}_{60}$ ) covering most of the spectrum between 300 and $800 \mathrm{~nm}$ (see Fig. 42). The stack design is inverted, i.e., the ITO is used as electron contact through a thin $\mathrm{n}$-doped $\mathrm{C}_{60}$, thus demonstrating one degree of freedom doping creates. The recombination contact between the subcells is realized using doped transport layers as described in section. An additional recombination contact is used below the Al-electrode due to processing reasons, but is not essential, as it has successfully been removed in later stack designs.

The importance of the doped transparent spacer is illustrated in the bottom graph in Fig. 43 showing the optical

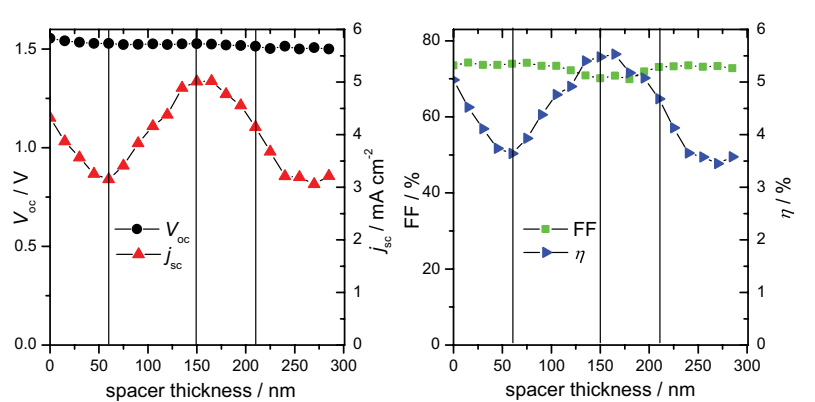

Figure 44 The parameters extracted from the $I-V$ curves of tandem devices with a variation of the spacer layer thickness between 0 and $285 \mathrm{~nm}$. All measurements were carried out at the same, but not mismatch corrected intensity of $98 \mathrm{~mW} \mathrm{~cm}^{-2}$ (Si reference diode). The vertical lines indicate the values used for the simulations shown in Fig. 43.

simulations of the stack. The curves illustrates how much the absorption of the F4-ZnPc based subcell varies as function of the spacer thickness even though all other layers were kept the same, as they were made in the same process. This directly translates into the experimental results presented in Fig. 44 that shows the characteristic parameters of the IV curves versus the thickness of the transparent spacer. $V_{\mathrm{oc}}$ is the sum of the voltages generated by the subcells and FF is above $70 \%$ all the time. Furthermore, $V_{\mathrm{oc}}$ and $\mathrm{FF}$ remain nearly constant through the complete spacer range indicating a very well working recombination contact.

The main difference for varying spacer thicknesses is observed in $j_{\mathrm{sc}}$ and subsequently in $\eta$ of the tandem device. As shown by the optical simulations in Fig. 43 (bottom), the variations in $j_{\mathrm{sc}}$ are a clear consequence of the thin film optics. Starting without spacer, both subcells are just about too thick to fit into the first interference maximum. With increasing spacer thickness the red absorbing subcell (F4-ZnPc: $\mathrm{C}_{60}$ ) is shifted through an interference minimum of the wavelengths it absorbs (about $60 \mathrm{~nm}$ spacer thickness) before arriving at the second interference maximum at about $150-165 \mathrm{~nm}$ spacer thickness. Here, $j_{\mathrm{sc}}$ of the tandem device reaches its maximum and hence $\eta$ is maximized, too.

The optical simulations furthermore clearly show why it makes sense to put the red (lower optical gap) absorbing subcell in front of the green (higher optical gap) absorbing subcell in this example. The red interference maximum forms further away from the Al mirror than the green interference maximum. Given that the absorption bands of organic semiconductors can be made narrow and really complementary, both subcells are positioned where they absorb most.

Although tandem OSC heavily rely on interference effects, their performance under different angles of illumination seems to be hardly affected. For the example above, simulations showed that the absorption in both subcells is reduced in a nearly equal way for off-angle illumination and thus, no subcell becomes dominantly current limiting. This was also experimentally confirmed [159]. 
This optical optimization using doped and transparent spacer layers is one of the key stack components for the world record OSC that are based on vacuum deposited small molecules [152].

5.3 Conclusions Doping of organic materials has several distinct advantages for OSC, the main being that using doped transparent transport layers to sandwich the photovoltaic active layers allows to create stacks with energy levels which closely resemble the ideal solar cell structure as proposed by Würfel [163]. Furthermore, doping of transport layers creates many degrees of freedom in the choice of electrode materials, layer sequence, and optical optimization, because it allows controlling the position of the Fermi levels. To sum up, doped organic layer create a very versatile basis for both investigation of fundamental processes in OSCs and the systematic optimization of single and tandem OSC.

6 Summary and outlook Organic semiconductors are already used in displays for handheld devices and will enter several other commercial markets soon. Organic doping has played an important role in the success of organic devices. In particular, organic doping helps to enhance the efficiency of optoelectronic devices and to improve the reproducibility of device processing. However, the precise mechanism of doping is still under scientific discussion and a detailed microscopic understanding of doping will help to design more efficient dopants in the future. Furthermore, despite its success in optoelectronic devices, doping is less commonly used in organic solar cells and organic transistors, leaving many opportunities for future research.

Acknowledgements The authors would like to thank Christiane Falkenberg, Torben Menke, Max Tietze, and Hans Kleemann for proof reading, Caroline Murawski and Christian Körner for the graphical abstract, and Caroline Murawski for the cover image. Most results discussed in the text are based on the results of Martin Pfeiffer, Jan Blochwitz-Nimoth, Christiane Falkenberg, Ronny Timmreck, BASF SE, Heliatek GmbH, Roland Gresser, Melanie Lorenz, Rico Schueppel, Sebastian Reineke, Rico Meerheim, Mauro Furno, Selina Olthof, Thomas Rosenow, and Simone Hofmann, which is gratefully acknowledged. We would also like to thank the Eurpean Comission, BMBF, DFG, Novaled AG, and Heliatek GmbH for funding.

\section{References}

[1] N. Karl, Defect Control in Semiconductors, Vol. II (North Holland, Amsterdam, 1990).

[2] L. Hung and C. Chen, Mater. Sci. Eng. R 39(5-6), 143-222 (2002).

[3] D. Berner, H. Houili, W. Leo, and L. Zuppiroli, Phys. Status Solidi A 202(1), 9-36 (2005).

[4] M. C. Gather, A. Köhnen, and K. Meerholz, Adv. Mater. 23(2), 233-248 (2011).

[5] Chem. Mater. 16(23) (2004), Special Issue on Organic Electronics.
[6] A. C. Arias, J. D. MacKenzie, I. McCulloch, J. Rivnay, and A. Salleo, Chem. Rev. 110(1), 3-24 (2010).

[7] P. Peumans, A. Yakimov, and S. R. Forrest, J. Appl. Phys. 93(7), 3693-3723 (2003).

[8] MRS Bull. 30(1), (2005). Bull MRS, Special Issue: Organic Based Photovoltaics.

[9] A. W. Hains, Z. Liang, M. A. Woodhouse, and B. A. Gregg, Chem. Rev. 110, 6689-6735 (2010).

[10] C. W. Tang, Appl. Phys. Lett. 48(2), 183-185 (1986).

[11] C. Tang and S. VanSlyke, Appl. Phys. Lett. 51, 913 (1987).

[12] M. Pfeiffer, A. Beyer, T. Fritz, and K. Leo, Appl. Phys. Lett. 73, 3202 (1998).

[13] X. Zhou, M. Pfeiffer, J. Blochwitz, A. Werner, A. Nollau, T. Fritz, and K. Leo, Appl. Phys. Lett. 784, 410-412 (2001).

[14] J. Blochwitz, T. Fritz, M. Pfeiffer, K. Leo, D. M. Alloway, P. A. Lee, and N. R. Armstrong, Org. Electron. 2, 97 (2001).

[15] B. Mnnig, M. Pfeiffer, A. Nollau, X. Zhou, and K. Leo, Phys. Rev. B 64(195), 208 (2001).

[16] A. Nollau, M. Pfeiffer, T. Fritz, and K. Leo, J. Appl. Phys. 87, 4340 (2000).

[17] A. Werner, F. Li, K. Harada, M. Pfeiffer, L. Fritz, K. Leo, and S. Machill, Adv. Funct. Mater. 14(3), 255-260 (2004).

[18] A. Werner, F. Li, K. Harada, M. Pfeiffer, T. Fritz, and K. Leo, Appl. Phys. Lett. 82(25), 4495-4497 (2003).

[19] F. Li, A. Werner, M. Pfeiffer, K. Leo, and X. Liu, J. Phys. Chem. B 108, 17076 (2004).

[20] K. Harada, A. G. Werner, M. Pfeiffer, C. J. Bloom, C. M. Elliott, and K. Leo, Phys. Rev. Lett. 94, 036601 (2005).

[21] Y. Yamamoto, K. Yoshino, and Y. Inuishi, J. Phys. Soc. Jpn. 47(6), 1887-1891 (1979).

[22] G. Parthasarathy, C. Shen, A. Kahn, and S. Forrest, J. Appl. Phys. 89(9), 4986-4992 (2001).

[23] J. Kido and T. Matsumoto, Appl. Phys. Lett. 73, 2866 (1998).

[24] J. Endo, T. Matsumoto, and J. Kido, Jpn. J. Appl. Phys., Part 2-Lett. 41(3B), L358-L360 (2002).

[25] D. Kearns, G. Tollin, and M. Calvin, J. Chem. Phys. 32(4), 1020-1025 (1960).

[26] M. Maitrot, G. Guillaud, B. Boudjema, J. Andre, and J. Simon, J. Appl. Phys. 60, 2396 (1986).

[27] J. Andre, J. Simon, R. Even, B. Boudjema, G. Guillaud, and M. Maitrot, Synth. Met. 18(1-3), 683-688 (1987).

[28] T. Marks, Science 227, 881 (1985).).

[29] E. Lous, P. Blom, L. Molenkamp, and D. Deleeuw, Phys. Rev. B 51(23), 17251-17254 (1995).

[30] M. Pfeiffer, T. Fritz, J. Blochwitz, A. Nollau, B. Plönnigs, A. Beyer, and K. Leo, Adv. Solid State Phys. 39, 77 (1999).

[31] K. Harada, M. Riede, K. Leo, O. R. Hild, and C. M. Elliott, Phys. Rev. B 77(19), 195212 (2008).

[32] S. Olthof, W. Tress, R. Meerheim, B. Lüssem, and K. Leo, J. Appl. Phys. 106(10), 103711 (2009).

[33] J. Blochwitz, M. Pfeiffer, T. Fritz, and K. Leo, Appl. Phys. Lett. 73, 729 (1998).

[34] J. Huang, M. Pfeiffer, A. Werner, J. Blochwitz, K. Leo, and S. Liu, Appl. Phys. Lett. 80(1), 139-141 (2002).

[35] X. Zhou, M. Pfeiffer, J. Huang, J. Blochwitz-Nimoth, D. Qin, A. Werner, J. Drechsel, B. Maennig, and K. Leo, Appl. Phys. Lett. 81, 922 (2002).

[36] J. Blochwitz, M. Pfeiffer, M. Hofmann, and K. Leo, Synth. Met. 127, 169-173 (2002).

[37] G. He, M. Pfeiffer, K. Leo, M. Hofmann, J. Birnstock, R. Pudzich, and J. Salbeck, Appl. Phys. Lett. 85, 3911 (2004). 
[38] Q. Huang, K. Walzer, M. Pfeiffer, V. Lyssenko, G. He, and K. Leo, Appl. Phys. Lett. 88, 113515 (2006).

[39] R. Meerheim, K. Walzer, M. Pfeiffer, and K. Leo, Appl. Phys. Lett. 89, 061111 (2006).

[40] S. Reineke, F. Lindner, G. Schwartz, N. Seidler, and K. Walzer, B. Lüssem, K. Leo, Nature 459, 234 (2009).

[41] M. Pfeiffer, A. Beyer, B. Plönnigs, A. Nollau, T. Fritz, K. Leo, D. Schlettwein, and S. Hiller, and D. Wöhrle, Sol. Energy Mater. Sol. Cells 63, 83 (2000).

[42] D. Gebeyehu, B. Maennig, J. Drechsel, K. Leo, and M. Pfeiffer, Sol. Energy Mater. Sol. Cells 79(1), 81-92 (2003).

[43] J. Drechsel, B. Maennig, F. Kozlowski, M. Pfeiffer, K. Leo, and H. Hoppe, Appl. Phys. Lett. 86, 244102 (2005).

[44] K. Schulze, C. Uhrich, R. Schueppel, K. Leo, M. Pfeiffer, E. Brier, E. Reinold, and P. Baeuerle, Adv. Mater. 18(21), 2872-2875 (2006).

[45] C. Uhrich, D. Wynands, S. Olthof, M. K. Riede, K. Leo, S. Sonntag, B. Maennig, and M. Pfeiffer, J. Appl. Phys. 104(4), 043107 (2008)

[46] D. Wynands, M. Levichkova, K. Leo, C. Uhrich, G. Schwartz, D. Hildebrandt, M. Pfeiffer, and M. Riede, Appl. Phys. Lett. 97, 073503 (2010).

[47] K. Walzer, B. Maennig, M. Pfeiffer, and K. Leo, Chem. Rev. 107, 1233 (2007).

[48] M. Pfeiffer, K. Leo, X. Zhou, J. Huang, M. Hofmann, A. Werner, and J. Blochwitz-Nimoth, Org. Electron. 4, 89 (2003).

[49] C. Adachi, R. Kwong, and S. Forrest, Org. Electron. 2, 37 (2001).

[50] M. Yoshida, A. Fujii, Y. Ohmori, and K. Yoshino, Appl. Phys. Lett. 69(6), 734-736 (1996).

[51] D. Ivory, G. Miller, J. Sowa, L. Shacklette, R. Chance, and R. Baughman, J. Chem. Phys. 71(3), 1506-1507 (1979).

[52] M. G. Ramsey, D. Steinmüller, and F. P. Netzer, Phys. Rev. B 42, 5902-5905 (1990).

[53] Y. Itoh, N. Tomikawa, S. Kobayashi, and T. Minato, Extended Abstracts, The 51th Autumn Meeting, Japan Society of Applied Physics, Morioka, Japan, 1990, p. 1040

[54] J. Kido, K. Nagai, and Y. Okamoto, IEEE Trans. Electron Devices 40, 1342-1344 (1993).

[55] T. Mori, H. Jujikawa, S. Tokito, and Y. Taga, Appl. Phys. Lett. 73, 2763 (1998).

[56] L. S. Hung, C. W. Tang, and M. G. Mason, Appl. Phys. Lett. 70(2), 152-154 (1997).

[57] M. G. Mason, C. W. Tang, L. S. Hung, P. Raychaudhuri, J. Madathil, D. J. Giesen, L. Yan, Q. T. Le, Y. Gao, S. T. Lee, L. S. Liao, L. F. Cheng, W. R. Salaneck, D. A. dos Santos, and J. L. Bredas, Appl. Phys. 89(5), 2756-2765 (2001)

[58] D. Grozea, A. Turak, X. D. Feng, Z. H. Lu, D. Johnson, and R. Wood, Appl. Phys. Lett. 81(17), 3173-3175 (2002).

[59] B. W. D'Andrade, S. Forrest, and A. Chwang, Appl. Phys. Lett. 83, 3858 (2003).

[60] C. J. Brabec, V. Dyakonov, J. Parisi, and N. S. Sariciftci, Organic Photovoltaics (Springer-Verlag, Berlin, Heidelberg, 2003).

[61] S. Tanaka, K. Kanai, E. Kawabe, T. Iwahashi, T. Nishi, Y. Ouchi, and K. Seki, Jpn. J. Appl. Phys. 44, 3760-3763 (2005)

[62] C. K. Chan, F. Amy, Q. Zhang, S. Barlow, S. Marder, and A. Kahn, Chem. Phys. Lett. 431(1-3), 67-71 (2006).
[63] C. J. Bloom, C. M. Elliott, P. G. Schroeder, C. B. France, and B. A. Parkinson, J. Phys. Chem. 107(13), 2933-2938 (2003).

[64] F. A. Cotton, N. E. Gruhn, J. Gu, P. Huang, D. L. Lichtenberger, C. A. Murillo, L. O. Van Dorn, and C. C. Wilkinson, Science 298, 1971-1974 (2002).

[65] F. A. Cotton, J. P. Donahue, D. L. Lichtenberger, C. A. Murillo, and D. Villagrán, J. Am. Chem. Soc. 127(31), 10808-10809 (2005).

[66] P. Wellmann, Lebensdauer und Stabilität elektrisch dotierter OLEDs, Dissertation, TU Dresden, 2009.

[67] T. Menke, D. Ray, S. Olthof, J. Meiss, K. Leo, and M. Riede, Appl. Phys. Lett. 100, 093304 (2012).

[68] C. Chan, E. G. Kim, J. L. Bredas, and A. Kahn, Adv. Funct. Mater. 16(6), 831-837 (2006).

[69] F. Li, M. Pfeiffer, A. Werner, K. Harada, K. Leo, N. Hayashi, K. Seki, X. Liu, and X. D. Dang, J. Appl. Phys. 100(2), 023716 (2006).

[70] E. S. Lewis, J. M. Perry, and R. H. Grinstein, J. Am. Chem. Soc. 92(4), 899-905 (1970).

[71] Y. M. A. Naguib, S. G. Cohen, and C. Steel, J. Am. Chem. Soc. 108(1), 128-133 (1986).

[72] S. Yamamoto, T. Sakurai, L. Yingjin, and Y. Sueishi, Phys. Chem. Chem. Phys. 1, 833-837 (1999).

[73] P. Wei, J. H. Oh, G. Dong, and Z. Bao, J. Am. Chem. Soc. 132(26), 8852-8853 (2010).

[74] P. Wei, T. Menke, B. D. Naab, K. Leo, M. Riede, and Z. Bao, J. Am. Chem. Soc. 134, 3999-4002 (2012).

[75] B. Maennig, J. Drechsel, D. Gebeyehu, F. Kozlowski, A. Werner, F. Li, S. Grundmann, S. Sonntag, M. Koch, K. Leo, M. Pfeiffer, H. Hoppe, D. Meissner, N. Sariciftci, I. Riedel, V. Dyakonov, and J. Parisi, Appl. Phys. A 79, 1 (2004).

[76] M. Riede, T. Mueller, W. Tress, R. Schueppel, and K. Leo, Nanotechnology 19, 424001 (2008).

[77] S. Guo, S. B. Kim, S. K. Mohapatra, Y. Qi, T. Sajoto, A. Kahn, S. R. Marder, and S. Barlow, Adv. Mater. 24, 699703 (2012).

[78] E. O. Fischer and H. Wawersik, J. Organomet. Chem. 5(6), 559-567 (1966).

[79] K. Yamashita, Y. Kunugi, Y. Harima, and A. N. Chowdhury, Jpn. J. Appl. Phys. 34, 3794-3797 (1995).

[80] S. Hamm and H. Wachtel, J. Chem. Phys. 103(24), 10689 10695 (1995).

[81] W. Shockley, Bell Syst. Tech. J. 28, 435-489 (1949).

[82] C. van Berkel, M. J. Powell, A. R. Franklin, and I. D. French, J. Appl. Phys. 73(10), 5264-5268 (1993).

[83] M. A. Kroon and R. A. C. M. M. van Swaaij, J. Appl. Phys. 90(2), 994-1000 (2001).

[84] H. Tsuji, C. Mitsui, Y. Sato, and E. Nakamura, Adv. Mater. 21(37), 3776-3779 (2009).

[85] C. Cai, S. J. Su, T. Chiba, H. Sasabe, Y. J. Pu, K. Nakayama, and J. Kido, Org. Electron. 12(5), 843-850 (2011).

[86] S. Hamwi, T. Riedl, and W. Kowalsky, Appl. Phys. Lett. 99(5), 053301 (2011).

[87] H. Kleemann, R. Gutierrez, F. Lindner, S. Avdoshenko, P. D. Manrique, B. Lussem, G. Cuniberti, and K. Leo, Nano Lett. 10(12), 4929-4934 (2010).

[88] A. Bernanose and J. Brit, Appl. Phys. Suppl. 4, S54-S56 (1955).

[89] W. Helfrich and Wg. Schneider, Phys. Rev. Lett. 14(7), 229 (1965). 
[90] D. Williams and M. Schadt, Proc. Inst. Electr. Electron. Eng. 58(3), 476 (1970).

[91] F. Kampas and M. Gouterman, Chem. Phys. Lett. 48(2), 233-236 (1977).

[92] P. Vincett, W. Barlow, R. Hann, and G. Roberts, Thin Solid Films 94(2), 171-183 (1982).

[93] M. Baldo, D. O'Brien, Y. You, S. Shoustikov, S. Sibley, M. Thompson, and S. Forrest, Nature 395, 151 (1998).

[94] R. Meerheim, K. Walzer, G. He, M. Pfeiffer, and K. Leo, Proc. SPIE 6192, 22 (2006).

[95] R. Meerheim, M. Furno, S. Hofmann, B. Luessem, and K. Leo, Appl. Phys. Lett. 97(25), 253305 (2010).

[96] R. Meerheim, B. Luessem, and K. Leo, Proc. IEEE 97(9), 1606-1626 (2009).

[97] N. C. Greenham, R. H. Friend, and D. D. C. Bradley, Adv. Mater. 6, 491 (1994).

[98] M. Baldo, S. Lamansky, P. Burrows, M. Thompson, and S. Forrest, Appl. Phys. Lett. 75(1), 4-6 (1999).

[99] T. Kobayashi, N. Ide, N. Matsusue, and H. Naito, Jpn. J. Appl. Phys., Part 1 44(4A), 1966-1969 (2005).

[100] K. Meerholz and D. Müller, Adv. Funct. Mater. 11, 251 (2001).

[101] R. Meerheim, S. Scholz, S. Olthof, G. Schwartz, S. Reineke, K. Walzer, and K. Leo, J. Appl. Phys. 104, 014510 (2008).

[102] N. Chopra, J. Lee, Y. Zheng, S. H. Eom, J. Xue, and F. So, Appl. Phys. Lett. 93(14), 143307 (2008).

[103] M. Furno, R. Meerheim, M. Thomschke, S. Hofmann, B. Luessem, and K. Leo, Proc. SPIE 7617, 761716 (2010).

[104] S. Hofmann, M. Thomschke, P. Freitag, M. Furno, B. Luessem, and K. Leo, Appl. Phys. Lett. 97(25), 253308 (2010).

[105] B. Lüssem, S. Reineke, T. Rosenow, G. Schwartz, and K. Leo, Proc. SPIE 7617, 761712 (2010).

[106] Y. Sun, N. Giebink, H. Kanno, B. Ma, M. Thompson, and S. Forrest, Nature 440, 908 (2006).

[107] G. Schwartz, M. Pfeiffer, S. Reineke, K. Walzer, and K. Leo, Adv. Mater. 19, 3672 (2007).

[108] G. Schwartz, S. Reineke, T. C. Rosenow, K. Walzer, and K. Leo, Adv. Funct. Mater. 19(9), 1319-1333 (2009).

[109] G. Schwartz, S. Reineke, K. Walzer, and K. Leo, Appl. Phys. Lett. 92, 053311 (2008).

[110] J. Y. Lee, S. T. Connor, Y. Cui, and P. Peumans, Nano Lett 8, 689-692 (2008).

[111] J. I. Lee, H. Y. Chu, J. Lee, and S. M. Chung, J. Nanosci. Nanotechnol. 8, 5602-5605 (2008).

[112] M. E. Kondakova, D. J. Giesen, J. C. Deaton, L. S. Liao, T. D. Pawlik, D. Y. Kondakov, M. E. Miller, T. L. Royster, and D. L. Comfort, SID Symp. Digest Tech. Papers 39, 219 (2008).

[113] T. C. Rosenow, M. Furno, S. Reineke, S. Olthof, B. Luessem, and K. Leo, J. Appl. Phys. 108(11), 113113 (2010).

[114] G. Chamberlain, Sol. Cells 8(1), 47-83 (1983).

[115] M. Hiramoto, H. Fujiwara, and M. Yokoyama, Appl. Phys. Lett. 58(10), 1062-1064 (1991).

[116] K. Vandewal, K. Tvingstedt, A. Gadisa, O. Inganas, and J. V. Manca, Nature Mater. 8(11), 904-909 (2009).

[117] H. Hoppe and N. Sariciftci, J. Mater. Chem. 16(1), 45-61 (2006).

[118] A. Salleo, R. Kline, D. Delongchamp, and M. Chabinyc, Adv. Mater. 22, 3812-3838 (2010).

[119] M. A. Green, K. Emery, Y. Hishikawa, W. Warta, and E. D. Dunlop, Prog. Photovolt.: Res. Appl. 20, 606-614 (2012).
[120] B. P. Rand, J. Genoe, P. Heremans, and J. Poortmans, Prog. Photovolt. 15, 659-676 (2007).

[121] C. J. Brabec, Sol. Energy Mater. Sol. Cells 83(2-3), 273292 (2004).

[122] G. Dennler, M. C. Scharber, and C. J. Brabec, Adv. Mater. 21, 1323-1338 (2009).

[123] ASTM, ASTM Standard G173, Standard Tables for Reference Solar Spectral Irradiances: Direct Normal and Hemispherical on $37^{\circ}$ Tilted Surface, 2010.

[124] V. Shrotriya, G. Li, Y. Yao, T. Moriarty, K. Emery, and Y. Yang, Adv. Funt. Mater. 16(15), 2016-2023 (2006).

[125] G. F. Burkhard, E. T. Hoke, and M. D. McGehee, Adv. Mater. 22, 3293-3297 (2010).

[126] C. H. Seaman, Sol. Energy 29, 291-298 (1982).

[127] A. Cravino, P. Schilinsky, and C. J. Brabec, Adv. Funct. Mater. 17, 3906-3910 (2007).

[128] M. Hiramoto, M. Suezaki, and M. Yokoyama, Chem. Lett. 3(3), 327-330 (1990).

[129] Heliatek (2011), Heliatek achieves new world record for organic solar cells with certified $9.8 \%$ cell efficiency. http:// tinyurl.com/heliatek9-8.

[130] J. Rostalski and D. Meissner, Sol. Energy Mater. Sol. Cells 61, 87-95 (2000).

[131] N. S. Sariciftci, L. Smilowitz, A. J. Heeger, and F. Wudl, Science 258(5087), 1474-1476 (1992).

[132] S. E. Shaheen and C. J. Brabec, Appl. Phys. Lett. 78(6), 841-843 (2001).

[133] F. Padinger, R. S. Rittberger, and N. S. Sariciftci, Adv. Funct. Mater. 13(1), 85-88 (2003).

[134] K. Suemori, T. Miyata, M. Hiramoto, and M. Yokoyama, Jpn. J. Appl. Phys. 8A, 1014-1016 (2004).

[135] S. Pfuetzner, J. Meiss, A. Petrich, M. Riede, and K. Leo, Appl. Phys. Lett. 94, 253303 (2009).

[136] P. Würfel, Physics of Solar Cells: From Principles to New Concepts (Wiley-VCH, Weinheim, 2004).

[137] P. Peumans, V. Bulovic, and S. R. Forrest, Appl. Phys. Lett. 76(19), 2650-2652 (2000).

[138] C. Falkenberg, K. Leo, and M. K. Riede, J. Appl. Phys. 110, 124509 (2011).

[139] S. Barlow, Q. Zhang, B. R. Kaafarani, C. Risko, F. Amy, C. K. Chan, B. Domercq, Z. A. Starikova, M. Y. Antipin, T. V. Timofeeva, B. Kippelen, J. L. Bredas, A. Kahn, and S. R. Marder, Chem. Eur. J. 13, 12 (2007).

[140] W. Zhao and A. Kahn, J. Appl. Phys. 105, 123711 (2009).

[141] M. Furno, Osolemio (2010), unpublished.

[142] E. Centurioni, Appl. Opt. 44, $7532-7539$ (2005).

[143] T. Fritz, J. Hahn, and H. Böttcher, Thin Solid Films 170, 249 (1989).

[144] J. Meiss, N. Allinger, M. K. Riede, and K. Leo, Appl. Phys. Lett. 93(10), 103311 (2008).

[145] Solarmer (2010), Solarmer energy, Inc. breaks psychological barrier with $8.13 \%$ OPV efficiency.

[146] Konarka (2010), Konarka's Power Plastic Achieves World Record 8.3\% Efficiency Certification from National Energy Renewable Laboratory (NREL).

[147] L. J. A. Koster, V. D. Mihailetchi, and P. W. M. Blom, Appl. Phys. Lett. 88(9), 093511 (2006).

[148] M. C. Scharber, D. Mühlbacher, M. Koppe, P. Denk, C. Waldauf, A. J. Heeger, and C. J. Brabec, Adv. Mater. 18, 789-794 (2006).

[149] B. P. Rand, D. P. Burk, and S. R. Forrest, Phys. Rev. B 75(11), 115327 (2007). 
[150] G. Dennler, M. C. Scharber, T. Ameri, P. Denk, K. Forberich, C. Waldauf, and C. J. Brabec, Adv. Mater. 3, 579-583 (2008).

[151] T. Ameri, G. Dennler, C. Lungenschmied, and C. J. Brabec, Energy Environ. Sci. 2, 347-363 (2009).

[152] Heliatek (2012), Heliatek sets new world record efficiency of $10.7 \%$ for its organic tandem cell. http://tinyurl.com/ heliatek10-7.

[153] B. Yu, F. Zhu, H. Wang, G. Li, and D. Yan, J. Appl. Phys. 104(11), 114503 (2008).

[154] J. Y. Kim, K. Lee, N. E. Coates, D. Moses, T. Q. Nguyen, M. Dante, and A. J. Heeger, Science 317(5835), 222-225 (2007).

[155] J. Gilot, M. M. Wienk, and R. A. J. Janssen, Appl. Phys. Lett. 90(14), 143512 (2007).

[156] R. Timmreck, S. Olthof, M. K. Riede, and K. Leo, J. Appl. Phys. 108, 033108 (2010).

[157] S. Olthof, R. Timmreck, B. Luessem, M. Riede, and K. Leo, Appl. Phys. Lett. 100, 113302 (2012).

[158] R. Schueppel, R. Timmreck, N. Allinger, T. Mueller, M. Furno, C. Uhrich, K. Leo, and M. Riede, J. Appl. Phys. 107, 044503 (2010).

[159] M. Riede, C. Uhrich, J. Widmer, R. Timmreck, D. Wynands, G. Schwartz, W. M. Gnehr, D. Hildebrandt, A. Weiss, J. Hwang, S. Sundarraj, P. Erk, M. Pfeiffer, and K. Leo, Adv. Funct. Mater. 21, 3019-3028 (2011).

[160] J. Meiss, A. Merten, M. Hein, C. Schuenemann, S. Schäfer, M. Tietze, C. Uhrich, M. Pfeiffer, K. Leo, and M. Riede, Adv. Funct. Mater. 22, 405-419 (2012).
[161] R. Gresser, M. Hummert, H. Hartmann, K. Leo, and M. Riede, Chem. Eur. J. 17, 2939-2947 (2011).

[162] A. Yakimov and S. R. Forrest, Appl. Phys. Lett. 80(9), 1667-1669 (2002).

[163] P. Würfel, Chimia 61, 770-774 (2007).

[164] E. E. Aziz, A. Vollmer, S. Eisebitt, W. Eberhardt, P. Pingel, D. Neher, and N. Koch, Adv. Mater. 19, 3257-3260 (2007).

[165] I. Salzmann, G. Heimel, S. Duhm, M. Oehzelt, P. Pingel, B. M. George, A. Schnegg, K. Lips, R. P. Blum, A. Vollmer, and N. Koch, Phys. Rev. Lett. 108, 035502 (2012).

[166] A. Mityashin, Y. Olivier, T. Van Regemorter, C. Rolin, S. Verlaak, N. G. Martinelli, D. Beljonne, J. Cornil, J. Genoe, and P. Heremans, Adv. Mater. 24, 1535-1539 (2012).

[167] C. K. Chiang, C. R. Fincher, Jr., Y. W. Park, A. J. Heeger, H. Shirakawa, E. J. Louis, S. C. Gau, and A. G. MacDiarmid, Phys. Rev. Lett. 39, 1098-1101 (1977).

[168] R. McNeill, R. Siudak, J. H. Wardlaw, and D. E. Weiss, Aust. J. Chem. 16, 1056-1075 (1963).

[169] B. A. Baracus and D. E. Weiss, Aust. J. Chem. 16, 10761089 (1963).

[170] B. A. Bolto, R. McNeill, and D. E. Weiss, Aust. J. Chem. 16, 1090-1103 (1963).

[171] R. De Surville, M. Jozefowicz, L. T. Yu, J. Pepichon, and R. Buvet, Electrochim. Acta 13, 1451 (1968).

[172] J. E. McGinness, P. M. Corry, and P. Proctor, Science 183, 853-855 (1974). 